\title{
Transient motions of elasto-viscoplastic thixotropic materials subjected to an imposed stress field and to stress-based free-surface boundary conditions
}

\author{
Cassio M. Oishi ${ }^{\mathrm{a}}$, Roney L. Thompson ${ }^{\mathrm{b}, *}$, Fernando P. Martins ${ }^{\mathrm{a}}$ \\ a Departamento de Matemática e Computação, Faculdade de Ciências e Tecnologia, Universidade Estadual Paulista "Júlio de Mesquita \\ Filho", 19060-900 Presidente Prudente, SP, Brazil \\ ${ }^{\mathrm{b}}$ Dep. Mechanical Engineering, COPPE, Universidade Federal do Rio de Janeiro, Centro de Tecnologia, Ilha do Fundão, Rio de Janeiro, \\ RJ 21945-970, Brazil
}

\section{A R T I C L E I N F O}

\section{Article history:}

Received 16 April 2016

Revised 29 July 2016

Accepted 28 August 2016

Available online 29 September 2016

\section{Keywords:}

Elasto-viscoplastic thixotropic materials

Numerical solution

Finite difference marker and cell method

Transient computations

Free-surface boundary conditions

\begin{abstract}
A B S T R A C T
In the present work, we present an approach to handle transient flows of elastoviscoplastic thixotropic (EVPT) materials under the action of the gravity force field and subjected to free-surface boundary conditions that are stress-based. This kind of conditions are common in problems where the motion is unknown a priori, i.e. the motion is treated as a consequence while the forces are the cause. The model for the EVPT material employed is within the scope of a recently developed thermodynamic backbone for elastoviscoplastic thixotropic materials. The finite difference Marker and Cell method is used to investigate effects of elasticity, thixotropy, and plasticity varying the Weissenberg number, the dimensionless thixotropic equilibrium time, and the yield number, respectively. The Cartesian Poiseuille flow is used to test the main features of the numerical scheme. The evolution in time of an initially square and fully-structured block is captured. The structure level and shape evolutions of the block reveal the capability of the present numerical approach to handle the complexity of the material and the free-surface motion.
\end{abstract}

(c) 2016 Elsevier Ltd. All rights reserved.

\section{Introduction}

\subsection{General aspects}

Many materials exhibit thixotropic and other time-dependent characters. Examples are encountered in many industries such as oil, food, cosmetics, mining, etc. In fact, time-dependent materials reflect the fact that it takes a finite time for the microstructure to achieve a new state once the stress has changed to a new value. In this sense, every microstructured material can exhibit time-dependency. However, a system is classified as time-dependent if the time scale associated to microstructure change is comparable to the process time scale.

Important recent advances on the understanding of thixotropic and other time-dependent phenomena were reported after careful conduction of experiments. In this connection, many findings and discussions concerning the detection of

\footnotetext{
* Corresponding author.

E-mail address: rthompson@mecanica.coppe.ufrj.br (R.L. Thompson).
} 
aging (Nigmatullin, Nakanishi, Tran-Cong-Miyata, Tahara, \& Fukao, 2010), the yielding process (Balmforth, Frigaard, \& Ovarlez, 2014; Coussot, 2014; Denn \& Bonn, 2011), the avalanche effect (Coussot, Nguyen, Huynh, \& Bonn, 2002a; 2002b), the bifurcation phenomenon (Coussot et al., 2002a), shear-banding (Bautista et al., 2012; Fall, Paredes, \& Bonn, 2010; Olmsted, 2008), and others, became crucial on analyzing the time-dependent nature of these kinds of complex materials. Some important works in the literature (Coussot et al., 2002a; Fall et al., 2010; Moller, Fall, Chikkadi, Derks, \& Bonn, 2009; Møller, Mewis, \& Bonn, 2006) have shown that there are classes of materials where the yielding phenomenon takes place which are susceptible to time-dependency. In this sense, it is important to understand the connections between these phenomena.

In an attempt to follow these findings and aiming to predict the behavior of microstructured materials, a number of models has been proposed. Since time-dependent in general constitute a broad class of materials, the great majority of these models focus on the important sub-class of the thixotropic materials. Thixotropic materials are common time-dependent systems that have their structure level decreased by the action of stress but they re-built reversibly when stresses are removed. Although thixotropic models are available for quite a time, being employed in simulations to solve benchmark and, less commonly, applied problems, only recently the elastic character of thixotropic materials, associated to a more structured state, has been incorporated to viscoplastic thixotropic models. In this context, de Souza Mendes, Rajagopal, and Thompson (2013) developed a thermodynamic framework for EVPT materials where a maximization of the entropy production is performed after assumptions of how the elasto-viscoplastic-thixotropic material stores and dissipates energy in consonance to a general thermodynamic theory developed by Rajagopal and co-workers (e.g. Rajagopal \& Srinivasa, 1998a; 1998b; Rao \& Rajagopal, 2002; Rajagopal \& Srinivasa, 2004a; 2004b).

In many problems of practical interest EVPT materials are subjected to a stress field originated from body and contact forces. In this case, there are no boundary nor initial conditions which are based on flow rate or velocity profile. The motion is completely treated as a consequence, while the forces are the cause. From the philosophical point of view, a deep discussion on this matter of causality was conducted by Rajagopal and co-workers and used as insight to build new classes of constitutive equations (e.g. Rajagopal, 2011; Bustamante \& Rajagopal, 2011; Muliana, Rajagopal, \& Wineman, 2013; Gou, Mallikarjuna, Rajagopal, \& Walton, 2015).

There are important problems associated to geophysical flows of viscoplastic materials where the action of gravity coupled with free surface boundary conditions constitute a crucial aspect (see Ancey, 2007). Examples where this kind of coupling was investigated are: the dam-break problem (e.g. Ancey \& Cochard, 2009; Balmforth, Craster, Perona, Rust, \& Sassi, 2007); the slump test (e.g. Dubash, Balmforth, Slima, \& Cochard, 2009; Staron, Lagrée, Ray, \& Popinet, 2013), also referred to as the "fifty-cent" rheometer (e.g. Pashias, Boger, Summers, \& Glenister, 1996; Roussel \& Coussot, 2005); the uniform flow of a layer of fluid on an inclined plane (e.g. Chambon, Ghemmour, \& Naaim, 2014; Huynh, Roussel, \& Coussot, 2005); the flow of a fixed amount of material on an inclined plane (e.g. Coussot, Nguyen, Huynh, \& Bonn, 2002b; Hewitt \& Balmforth, 2013); among others.

\subsection{Viscoplastic time-dependent phenomena}

Careful reviews on thixotropic systems were performed in Barnes (1997); Mewis and Wagner (2009); Mujumdar, Beris, and Metzner (2002) and the reader is referred to these articles in order to get an overview of this phenomenon.

Thixotropy yield-stress materials exhibit remarkable differences when compared to ideal yield-stress ones (Coussot et al., 2002a; Fall et al., 2010). Yield-stress materials exhibit solid-like to liquid-like transition when a viscoplastic material that was in a free-stress state for a long time (fully structured state) is exposed to a stress level above the yield stress. In this case, the material starts an internal process that is associated to an evolution towards less structured states until it reaches an equilibrium structure configuration associated to the imposed stress. Thixotropic systems have a spontaneous "internal" build-up agent, like Brownian motion for example, responsible for the aging of the material even at rest in a stress free state, and a breakdown agent that acts as a rejuvenation source. The competition between these two sources leads to aging, rejuvenation, or equilibrium. When the stress level is above, but close to the yield stress, the break-up rate is low and the time dependency nature of the material is more pronounced.

A striking phenomenon where the thixotropic character of the material is unequivocally present is the so-called "avalanche effect", as discussed in Coussot et al. (2002a, 2002b). When a structured material is subjected to gravity forces on an inclined plane, above a certain angle, the stress overcomes the yield stress, starting a breakage process. When the material breaks, its viscosity decreases, what enhances the flow rate of the material, what, in turn, favors more breakage. Therefore, a continuous cyclic self-feeding process takes place, rendering the achievement of high distances at the end.

Shear banding or shear localization is also a phenomenon typical of viscoplastic materials where thixotropy plays an important role (Bautista et al., 2012; Fall et al., 2010; Olmsted, 2008; Ovarlez, Rodts, Chateau, \& Coussot, 2009). Shear banding occurs when similar stress levels lead to the coexistence of remarkably different shear rate levels. When the flow curve is non-monotonic, depending on the imposed variable, stress or shear-rate, different parts of the stress-shear-rate space are attainable or unattainable. In fact, there are parts of the flow curve that can be always unattainable, leading the necessity of the performance of a stability analysis approach. This phenomenon is related to the presence of a dynamic yield stress value which is lower than the static yield stress value. While the dynamic yield stress is usually defined as the stress value at the transition from liquid to solid behavior, the static yield stress is the stress value at the transition from solid to liquid behavior. 
Viscosity bifurcation is another viscoplastic phenomenon where thixotropy plays a fundamental role. Originally (see Coussot et al., 2002a), bifurcation behavior was detected in a shear-rate evolution in time for different levels of applied shear stress. For low values of applied stress, the shear rate develops a trajectory in time that ends in a negligible shear rate. For high stress values, this phenomenon can also be detected in a deformation versus time plotting where an almost constant plateau is reached for low stress values (Tarcha, Forte, Soares, \& Thompson, 2015). The difficulties in experimentally determining the yield stress of a material are known in the literature and are more pronounced in thixotropic and other time-dependent systems as discussed in Møller et al. (2006) and Soares, Thompson, and Machado (2013). The viscosity bifurcation phenomenon is becoming an important procedure to measure this entity.

\subsection{Thixotropic models}

Modeling thixotropic behavior is not an easy task. There are three approaches for modeling the change of the material structure in time. The first one is to consider an evolution equation for a direct structure quantity such as the number of bonds or links per unit volume in the material (Kee \& Fong, 1993). A second approach is to consider an evolution for a macroscopic quantity such as viscosity or fluidity (Bautista, de Santos, Puig, \& Manero, 1999; Sadequi, Khabazi, \& Sadeghy, 2011). The third and most commonly adopted approach is based on an entity generally called structure parameter, $\lambda$, conceived to represent the structure level of the material (Mujumdar et al., 2002). Depending on the material, this structure parameter can be related to different physical entities (Barnes, 1997). A crucial step is to establish the relation of macroscopic quantities such as viscosity and elastic modulus with the structure parameter. For a large variety of materials the levels of viscosity and elasticity decay when the material is being destructed. In this connection, the relation between viscosity and the structure parameter is direct, i.e. when one increases the other increases also. However, the relation between $\lambda$ and the elastic modulus depends on the model considered, as discussed in de Souza Mendes and Thompson (2012). This happens because depending on the model, the elastic mechanism can be in series with the structure viscosity or in parallel with this quantity. A kinetic equation that governs the evolution of this structure parameter is introduced in order to describe the non-equilibrium micro-structured states. This equation invariably obeys the following backbone

$$
\frac{d \lambda}{d t}=\dot{\lambda}_{f}-\dot{\lambda}_{d}
$$

where $\dot{\lambda}_{f}$ represents the rate of structure formation, while $\dot{\lambda}_{d}$ represents the rate of destruction of the bonds of the material. An equilibrium state is achieved when $\dot{\lambda}_{f}=\dot{\lambda}_{d}$. Depending on the model, different expressions are used to describe formation and destruction of micro-structure. One very often structure is represented by

$$
\frac{d \lambda}{d t}=k_{1}(1-\lambda)^{a}-k_{2} \lambda^{b} g(\dot{\gamma}),
$$

where $g(\dot{\gamma})$ is a general function of the shear rate and $k_{1}, k_{2}, a$, and $b$ are constant parameters.

Another similar backbone is to represent the evolution equation for the structure parameter using the difference between the current value of $\lambda$ and the equilibrium value, $\lambda_{e q}$, as a driving potential for structure changes (see Baravian, Quemada, \& Parker, 1996). Although there are some cases in which these two formulations are equivalent, this is not true for every evolution equation. This kind of evolution has, in general, the form given by

$$
\frac{d \lambda}{d t}=\frac{1}{t_{\text {eq }}} \operatorname{sign}\left(\lambda_{\text {eq }}-\lambda\right)\left|\lambda-\lambda_{\text {eq }}\right|^{\mathrm{n}_{\mathrm{c}}},
$$

where $t_{e q}$ is thixotropic equilibrium time, $\operatorname{sign}\left(\lambda_{\text {eq }}-\lambda\right)$ is positive when $\lambda_{e q} \geq \lambda$ and negative otherwise, and $n_{c}$ is a constant parameter.

The modeling of the aging process of a thixotropic system is generally obtained from an equation that is not affected by current values of stress or shear rate since this kind of material is such that, at rest, is capable of a fully restructuring.

A worth noticing fact is related to the intrinsic choice of the structure breaking agent (de Souza Mendes \& Thompson, 2012; Yziquel, Carreau, Moan, \& Tanguy, 1999). Depending on the form of the break down term, the agent responsible for the destruction is chosen. By far, the most common path used to model the rejuvenation of the material is by considering a function that depends on the shear rate (see Barnes, 1997). One rare exception is to employ the power stress as destructing agent, as done in Bautista et al. (1999) modeling micellar solutions (see also López-Aguilar, Webster, Tamaddon-Jahromi, and Manero, 2015 for a recent work). In Marrucci, Titomanlio, and Sarti (1973) the authors propose that the stress should be the entity responsible for the decrease in the structure level of the material, in the context of entangled systems. Yziquel et al. (1999) found that the stress power is the best option, although they have not considered the second invariant of the stress in their comparison. In de Souza Mendes and Thompson (2012) it is discussed some disadvantages of considering a function of the shear rate as the break down agent. This viewpoint is also shared in Van Der Geest, Guersoni, Merino-Garcia, and Bannwart (2015). The main problem concerns the prediction of the rejuvenation in an undeformed material at rest. In many cases, it is the destruction of the material that causes motion (avalanche). Hence, it is necessary to have a break down term that does not vanish in the absence of motion. The IKH model of Dimitriou and McKinley (2014) uses a plastic strain rate, $\dot{\gamma}_{p}$, in the place of $\dot{\gamma}$ in Eq. (2), however, $\dot{\gamma}_{p}$ is defined with a stress driven potential, what in turn connects to the current stress value the role of breaking agent. A similar rationale can be applied to the model of Hewitt and Balmforth (2013) where the 
shear rate in the breakdown term is substituted by the stress value corresponding to the flow curve relation. It is worth mentioning that this model is able to capture the bifurcation phenomenon.

Although there are time-dependent models related to viscoelastic phenomena such as the one explored in Marrucci et al. (1973), and more recently in Khajehsaeid, Arghavani, Naghdabadi, and Sohrabpour (2014), we are focusing our attention to model thixotropic phenomena associated to viscoplasticity. In this connection, it is a common approach to consider, not only the viscosity, but also, the yield stress as a parameter that depends on the structure level (Nguyen \& Boger, 1985). As discussed by de Souza Mendes and Thompson (2012) this is inconsistent with the original concept of the yield stress, which is a property associated to the fully-structured state solely.

The development of elasto-viscoplastic-thixotropic models is more recent and can be roughly divided into two kinds: one that includes viscoplastic and thixotropic effects on a original viscoelastic model (Bautista et al., 1999; Quemada, 1999; Yziquel et al., 1999) and the other that adds elasticity and thixotropy on a original viscoplastic model (Dullaert \& Mewis, 2006; Mujumdar et al., 2002). An analysis of these types of modeling was conducted by Mewis and Wagner (2009) and de Souza Mendes and Thompson (2012).

One important breakthrough in thixotropic modeling was proposed in de Souza Mendes (2009). In this work, the stress is considered the destructing agent and the flow curve is an additional information that is used to calculate the equilibrium microstructure state. Another important assumption was made with respect to the evolution of a neutral configuration. It evolves in such a way that changes in the elastic stress are related to changes in elastic strain while changes in the neutral configuration are due to changes in the structure level. With this hypothesis, the final shear equation is of the same form as the Maxwell equation. Besides that, a decreasing function of the shear modulus is proposed. The model is conceived for an apparent-yield-stress fluid. In this same structure, de Souza Mendes (2011) extended the model including a retardation viscosity and (de Souza Mendes \& Thompson, 2013) included classical yield-stress materials in a single unified backbone. An important theoretical step was introduced by de Souza Mendes et al. (2013) where a full 3-D model based on thermodynamics considerations was deduced. It is worth noticing that a different thermodynamic analysis for elastoviscoplastic materials was conducted by dos Santos, Ramos, and Rossi (2015) and that this backbone can be generalized to EVPT systems.

\subsection{Relevant numerical works}

Numerical works that deal with the simulation of viscoplastic materials are reasonably common in the literature and are basically explored in two branches: the augmented Lagrangian method (see Glowinsky \& Wachs, 2011; Vola, Boscardin, \& Latché, 2003) and the viscosity regularization method (see Frigaard \& Nouar, 2005; Mitsoulis \& Huilgol, 2004). The former is used when the problem requires an accurate method to determine the position of the yield surface on a (true-)yield-stress material and is based on a variational formulation of the momentum equation together with an optimization algorithm. The latter is used when the material can be considered an apparent-yield-stress material or the position of the yield surface can be roughly located and are based on a smooth viscosity function with a finite, but large, value when the shear rate vanishes. Recently, an alternative optimization method for dealing with yield stress fluid flows has been proposed by Bleyer, Maillard, de Buhan, and Coussot (2015). In the context of numerical simulations for elasto-viscoplastic materials, few methods have been proposed, for instance (Cheddadi \& Saramito, 2013; dos Santos, Frey, Naccache, \& de Souza Mendes, 2014). An additional difficulty that can be found in some cases is the simulation of viscoplastic flows in the presence of free surface boundaries, and some physical insights were presented in Mitsoulis (2007) and Ancey and Cochard (2009).

Numerical works that deal with the simulation of the flow of thixotropic materials are still scarce in the literature. Due to inherent complexities and low convergence of augmented Lagrangian method, the regularized method is generally chosen when thixotropic effects are added to the model. One of the first examples of simulations of thixotropic materials as applied to biological systems was conducted by Bottino (1998), where a network model was developed to mimic cell behavior. In Derksen and Regulagadda (2009) the authors used a lattice-Boltzmann method to model a thixotropic viscoplastic material of Bingham type. The kinetic equation for the structure parameter was a very common one, where the shear-rate is considered the destructing agent. Potanin (2010) conducted 3D simulations of a thixotropic material in standard rheometric geometries comparing the results with the same experimental device. The kinetic equation for the structure parameter obeyed Eq. (3), with $t_{e q}$ and $\lambda_{e q}$ dependent on the shear rate. He used the commercial software FLUENT, based on a Finite Volume discretization method. The comparison between a thixotropic and non-thixotropic materials was conducted in Alexandrou, Florides, and Georgiou (2013) on the squeeze flow. They used a Lagrangean Gelerkin method, with the points of the interface moving with its calculated velocity. Two kinds of boundary conditions were imposed at the top: constant velocity and constant force. Among the few examples of numerical works on elasto-viscoplastic thixotropic materials are the works of Fonseca, Frey, Naccache, and de Souza Mendes (2013), where a Maxwell based viscoelastic model is used as a basis for the inclusion of viscoplastic and thixotropic effects and (Link, Frey, Thompson, Naccache, \& de Souza Mendes, 2015; López-Aguilar et al., 2015) where an Oldroyd-B based viscoelastic model is the original backbone.

\subsection{Objective and scope}

Many applied problems involving thixotropic and other time-dependent materials associated to viscoplasticity are intrinsically transient and are subjected to the action of gravity coupled with free-surface boundary conditions. Besides that, these 
materials can exhibit relevant elastic effects, especially in the solid-like regime. This class of problems combines the complexity of the material with the complexity of the flow. This combination constitutes a challenging for numerical schemes in order to provide robust solutions, able to fulfill the needs of geophysical and industrial processes.

The main objectives of this work are two-folded: i) apply a robust numerical scheme for solving elasto-viscoplastic thixotropic (EVPT) materials subjected to an imposed stress field and to free-surface boundary conditions, and ii) capture transient motions of these complex materials that to the authors best knowledge, were not yet reported in the literature. The scope of this work is delineated next.

In Section 2 the mathematical formulation is given. The governing equations, the constitutive model of the elastoviscoplastic thoxotropic material, as well as the boundary conditions are stressed. An important aspect of the present approach are the dimensionless versions of the previous mentioned equations, which are also provided in this section. Section 3 addresses how the Marker-And-Cell-type formulation is adapted to the complex model considered. In Section 4 the two problems considered, namely the plane channel flow and the square block, are described. The results and discussions of the plane channel flow are provided in Section 5 while Section 6 provides the results and discussions of the square block. Finally, in Section 7 conclusions and suggestions for future work are given.

\section{Mathematical formulation}

\subsection{Governing equations}

For the isothermal motion of an incompressible material, the governing equations for mass and momentum are given by

$$
\begin{aligned}
& \nabla \cdot \mathbf{u}=0, \\
& \rho\left[\frac{\partial \mathbf{u}}{\partial t}+\nabla \cdot(\mathbf{u u})\right]=\nabla \cdot \boldsymbol{\sigma}+\rho \mathbf{g},
\end{aligned}
$$

where

$$
\sigma=-p \mathbf{I}+\boldsymbol{\tau}^{N}+\boldsymbol{\tau}^{M},
$$

and $\mathbf{g}$ is the acceleration of gravity used in free surface problems. In Eq. (6), $\boldsymbol{\tau}^{N}=2 \eta_{\infty} \mathbf{D}$ where $\mathbf{D}$ is the rate of deformation tensor and $\eta_{\infty}$ is the viscosity related to the fully unstructured material. In addition, $\boldsymbol{\tau}^{M}$ is a non-Newtonian part of the stress tensor that obeys an upper convected Maxwell equation of the following form

$$
\frac{\partial \boldsymbol{\tau}^{M}}{\partial t}+\nabla \cdot\left(\mathbf{u} \tau^{M}\right)-\left[(\nabla \mathbf{u}) \cdot \boldsymbol{\tau}^{M}+\boldsymbol{\tau}^{M} \cdot(\nabla \mathbf{u})^{\mathbf{T}}\right]=-\frac{G(\lambda)}{\eta(\lambda)} \boldsymbol{\tau}^{M}+2 G(\lambda) \mathbf{D},
$$

where $G(\lambda)$ and $\eta(\lambda)$ are the structure elastic modulus and structure viscosity. In the present work, the dependence of these material properties with respect to the structure parameter are given by

$$
\begin{aligned}
& \eta(\lambda)=\left[\left(\frac{\eta_{0}}{\eta_{\infty}}\right)^{\lambda}-1\right] \eta_{\infty}, \\
& G(\lambda)=G_{0} \exp \left[m\left(\frac{1}{\lambda}-1\right)\right],
\end{aligned}
$$

where $\eta_{0}$ and $G_{0}$ are the values of viscosity and elastic modulus in the fully structured state, respectively, and $m$ is a positive constant.

As described in de Souza Mendes et al. (2013), the above model belongs to a class of materials where the set of equations can be derived with assumptions on the form the material stores and dissipates energy that are compatible with the second law of thermodynamics.

\subsection{Flow curve}

The flow curve is generally obtained from a viscometric rheometry and constitutes a relation between the shear stress, $\tau$, and the deformation rate at steady-state conditions, i.e. equilibrium conditions. In the present work we will adopt the following equation that have shown to be useful to fit rheometric data

$$
\tau=\zeta_{j}(\dot{\gamma})\left(\tau_{y}+K \dot{\gamma}^{n}\right)+\eta_{\infty} \dot{\gamma},
$$

where $\tau_{y}$ is the apparent yield stress of the material; $K$ and $n$ are the consistency and exponent of the power-law term in the flow curve; and $\eta_{\infty}$ is the viscosity at high shear rates. The function $\zeta_{j}(\dot{\gamma})$ is a physical regularization factor used to model apparent yield stress materials. Options for $\zeta_{j}(\dot{\gamma})$ are stressed below (see de Souza Mendes and Thompson, 2013 for some of these and other options)

$$
\zeta_{1}(\dot{\gamma})=1-\exp \left(-\frac{\eta_{0}}{\tau_{y}} \dot{\gamma}\right)
$$


and

$$
\zeta_{2}(\dot{\gamma})=\frac{\eta_{0} \dot{\gamma}}{\sqrt{\left(\eta_{0} \dot{\gamma}\right)^{2}+\tau_{y}^{2}}}
$$

An alternative procedure to represent the flow curve is by using the bi-viscosity model shown below. This option can also be applied in the present case since we are considering apparent-yield-stress fluids. It is given by

$$
\tau= \begin{cases}\tau_{y}+K \dot{\gamma}^{n}+\eta_{\infty} \dot{\gamma} & \text { if } \tau \geq \tau_{y} \\ \eta_{0} \dot{\gamma} & \text { if } \tau<\tau_{y}\end{cases}
$$

This relation can be expressed by Eq. (10) with the following rule for $\zeta_{j}$

$$
\zeta_{3}(\dot{\gamma})=\left\{\begin{array}{lll}
1 & \text { if } & \tau \geq \tau_{y} \\
\frac{\left(\eta_{0}-\eta_{\infty}\right) \dot{\gamma}}{\tau_{y}+K \dot{\gamma}^{n}} & \text { if } & \tau<\tau_{y}
\end{array}\right.
$$

\subsection{Structure evolution}

The evolution for the structure parameter is an additional equation that is needed to be included in the formulation. From the perspective of Eq. (1), we need to specify the rate of formation and rate of destruction of micro-structure. In the present work we adopt the simplified equation stated in de Souza Mendes and Thompson (2012) (see also Baravian et al., 1996), in the version where the stress is identified as the destructing agent, i.e.

$$
\dot{\lambda}_{f}=c_{1}(1-\lambda),
$$

and

$$
\dot{\lambda}_{d}=c_{2} \lambda f(\sigma) .
$$

The function $f(\sigma)$ is determined from the equilibrium state, i.e. when rate of formation and rate of destruction are equal. Hence,

$$
c_{1}\left(1-\lambda_{\text {eq }}\right)=c_{2} \lambda_{\text {eq }} f(\sigma) \Rightarrow f(\sigma)=\frac{c_{1}}{c_{2}} \frac{1-\lambda_{\text {eq }}(\sigma)}{\lambda_{\text {eq }}(\sigma)},
$$

Therefore, the equation for the evolution of the structure parameter becomes independent of $c_{2}$. Recognizing that $c_{1}$ has dimensions of frequency, we can define $t_{e q}$ as the reciprocal of $c_{1}$. With some manipulation, the equation simplifies to

$$
\frac{\partial \lambda}{\partial t}+\nabla \cdot(\mathbf{u} \lambda)=\frac{1}{t_{e q}}\left(1-\frac{\lambda}{\lambda_{e q}(\sigma)}\right)
$$

where $\lambda_{e q}(\sigma)$ is a function of the current stress value and the form this function is calculated is described next. Two fundamental concepts are necessary to determine the current equilibrium value of the structure parameter. The first one is to identify the flow curve, as a set of steady-state points which is the locus of the attractor equilibrium points of the current state (see de Souza Mendes \& Thompson, 2012). The other, is that we are applying a von Misses criterion of yielding, based on the Maximum Distortion Energy Criterion (see von Mises, 1913). This implies that the second invariant of the deviatoric part of the stress must overcome the yield stress obtained in shear, here called $\tau_{y}{ }^{1}$, in order to trigger the breaking of the microstructure. In this sense, the attractor point of the current non-equilibrium state, among all points that belong to the flow curve, is the point corresponding to the current value of the square root of the second invariant of the deviatoric part of the stress (Link et al., 2015). In this sense, we can say that the relation between $\lambda_{\text {eq }}$ and the stress tensor reduces to $\lambda_{\text {eq }}=\hat{\lambda}_{\text {eq }}\left(\sigma_{\text {dev }}\right)$, where

$$
\sigma_{d e v}=\sqrt{\frac{1}{2} \operatorname{tr}\left[\left(\sigma-\frac{1}{3} \operatorname{tr}(\boldsymbol{\sigma}) \boldsymbol{I}\right)^{2}\right]} .
$$

In fact, $\lambda_{e q}$ is the equilibrium structure parameter obtained from the current von Mises stress, i.e. we use Eq. (10) by replacing $\tau$ by $\sigma_{d e v}$.

The approximated value in this iterative process is the equilibrium deformation rate value, $\dot{\gamma}_{\text {eq }}$, corresponding to the current stress tensor. The equilibrium viscosity is simply obtained by

$$
\eta_{e q}=\frac{\sigma_{d e v}}{\dot{\gamma}_{e q}}
$$

\footnotetext{
${ }^{1}$ In the solid mechanics literature it is not uncommon to call $\tau_{y}$ the yield stress of the uniaxial extensional motion. A factor of $\sqrt{3}$ differs the two quantities.
} 
Finally, we determine $\lambda_{e q}\left(\sigma_{\text {dev }}\right)$ corresponding to $\eta_{e q}$. This structure parameter is obtained through the inverse of the viscosity function expressed by Eq. (8)

$$
\lambda_{e q}=\frac{\ln \eta_{e q}-\ln \eta_{\infty}}{\ln \eta_{0}-\ln \eta_{\infty}} .
$$

In order to feed Eq. (18), we also need the value of the current structure parameter which is obtained with the corresponding expression

$$
\lambda=\frac{\ln \left[\eta(\lambda)+\eta_{\infty}\right]-\ln \eta_{\infty}}{\ln \eta_{0}-\ln \eta_{\infty}},
$$

where

$$
\eta(\lambda)+\eta_{\infty}=\frac{\sigma_{d e v}}{\dot{\gamma}}
$$

\subsection{Boundary conditions}

The adopted boundary conditions for solving Eqs. (4) and (5) are

$$
\mathbf{u}=\mathbf{0} \text { on } \partial \Omega_{d}
$$

and

$$
\boldsymbol{\sigma} \cdot \mathbf{n}=\mathbf{t} \text { on } \partial \Omega_{t},
$$

where $\partial \Omega_{d}$ is the boundary where the velocity $\mathbf{u}$ is prescribed and $\partial \Omega_{t}$ is the domain boundary for imposing of the traction condition. In Eq. (25), $\mathbf{n}$ denotes the outward unit normal vector to the boundary $\partial \Omega_{t}$ while $\mathbf{t}$ is the traction vector.

Notice that from Eq. (25) we can obtain the normal stress condition

$$
\mathbf{n} \cdot \boldsymbol{\sigma} \cdot \mathbf{n}^{T}=\mathbf{n} \cdot \mathbf{t}=F_{n} \quad \text { on } \partial \Omega_{t},
$$

and the tangential boundary condition

$$
\mathbf{m} \cdot \boldsymbol{\sigma} \cdot \mathbf{n}^{T}=\mathbf{m} \cdot \mathbf{t}=F_{m} \quad \text { on } \partial \Omega_{t},
$$

where $\mathbf{m}$ is the unit tangent vector while $F_{n}$ and $F_{m}$ are known functions according to the physical problem which will be simulated.

Concerning the boundary conditions for the structure parameter, it is desirable to solve the evolution equation for $\lambda$ at the boundaries, so that the stress solution at these locations can have a direct impact on the local structure parameter at the boundary. Although this strategy was well succeeded in one of the problems analyzed, the rejuvenation of a square initially fully structured, resolving $\lambda$ at the wall turned out to be unstable for the plane channel. In this case, convergence was achieved by using Neumann boundary conditions at walls. Some preliminary tests have shown that no significant difference between the two cases were found in the interior points, with the boundary condition change.

\subsection{Mathematical formulation in dimensionless form}

In this work we have adopted a dimensionless approach aligned to the philosophy described in Thompson and Soares (2016). In this connection, we have used the following dimensionless variables:

$$
t^{*}=t \frac{U_{c}}{L_{c}}, \quad \nabla^{*}=L_{c} \nabla, \quad \mathbf{u}^{*}=\frac{\mathbf{u}}{U_{c}}, \quad \boldsymbol{\tau}^{*}=\frac{\boldsymbol{\tau}}{\tau_{c}}, \quad p^{*}=\frac{p}{\rho U_{c}^{2}}, \quad \mathbf{g}^{*}=\frac{\mathbf{g}}{g} .
$$

It is common in the literature to deal with problems where the characteristic velocity scale, $U_{c}$, is a primitive quantity, like in the case where a flow rate or the velocity far from a solid body are imposed. In the present work, we define a characteristic stress as a primitive quantity, since we are analyzing problems where the boundary conditions are stress-based. In this connection, we have adopted $\tau_{c}=\sigma^{\max }(t=0)$, i.e. the characteristic stress is defined as the maximum stress intensity, $\sigma$, of the domain, evaluated at $t=0$. Therefore, $\tau_{c}$, as well as $L_{c}$, depends on the specific problem analyzed. No flow will happen if $\tau_{c} \leq \tau_{y}$, i.e. if the maximum stress intensity value does not overcome the yield stress. Accordingly, the characteristic velocity, $U_{c}$, is defined with respect to the maximum potential of destruction, the difference between the maximum stress at $t=0$ and the yield stress. From now on, we assume that $\tau_{c}$ exceeds $\tau_{y}$. Eq. (10) is used for determining $U_{c}$ in the simplified form where the regularized function is $\zeta_{j}(\dot{\gamma})=1$. Because of the power-law term that confers a non-linear character with respect to a characteristic velocity, $U_{c}$ is defined by the implicit equation

$$
\tau_{c}=\tau_{y}+K\left(\frac{U_{c}}{L_{c}}\right)^{n}+\eta_{\infty} \frac{U_{c}}{L_{c}}
$$

which can be made dimensionless using the characteristic stress, $\tau_{c}$, as follows

$$
1=\tau_{y}^{*}+K^{*}+\eta_{\infty}^{*}
$$


where

$$
\tau_{y}^{*}=\frac{\tau_{y}}{\tau_{c}}, \quad K^{*}=\frac{K}{\tau_{c}}\left(\frac{U_{c}}{L_{c}}\right)^{n}, \quad \eta_{\infty}^{*}=\frac{\eta_{\infty}}{\eta_{c}}
$$

and $\eta_{c} \equiv \tau_{c} L_{c} / U_{c}$.

The dimensionless versions of the conservation of mass and momentum as represented by Eqs. (4) and (5) are given by

$$
\nabla^{*} \cdot \mathbf{u}^{*}=0,
$$

and

$$
\frac{\partial \mathbf{u}^{*}}{\partial t^{*}}+\nabla^{*} \cdot\left(\mathbf{u}^{*} \mathbf{u}^{*}\right)=-\nabla p^{*}+\frac{1}{R e}\left(\eta_{\infty}^{*}\left(\nabla^{*}\right)^{2} \mathbf{u}^{*}+\nabla^{*} \cdot \boldsymbol{\tau}^{M *}\right)+\frac{1}{F r^{2}} \mathbf{g}^{*},
$$

respectively. The quantity $\eta_{\infty}^{*}$ is the dimensionless retardation viscosity and the Reynolds number, $R e$, and the Froude number, $F r$, are defined as

$$
R e=\frac{\rho U_{c}^{2}}{\tau_{c}}, \quad F r=\frac{U_{c}}{\sqrt{g L_{c}}} .
$$

In dimensionless form, the upper convected Maxwell Eq. (7) is rewritten as

$$
\frac{\partial \boldsymbol{\tau}^{M *}}{\partial t^{*}}+\nabla^{*} \cdot\left(\mathbf{u}^{*} \boldsymbol{\tau}^{M *}\right)-\left[\left(\nabla^{*} \mathbf{u}^{*}\right) \cdot \boldsymbol{\tau}^{M *}+\boldsymbol{\tau}^{M *} \cdot\left(\nabla^{*} \mathbf{u}^{*}\right)^{\mathbf{T}}\right]=\frac{f(\lambda)}{W i}\left[-\frac{1}{\eta^{*}(\lambda)} \boldsymbol{\tau}^{M *}+2 \mathbf{D}^{*}\right],
$$

where the Weissemberg number Wi, the dimensionless function $f(\lambda)$, the dimensionless rate-of-strain tensor, $\mathbf{D}^{*}$, and the dimensionless relaxation viscosity, $\eta^{*}(\lambda)$, are given by

$$
W i=\frac{\tau_{c}}{G_{0}}, \quad \mathbf{D}^{*}=\mathbf{D} \frac{L_{c}}{U_{c}}
$$

and

$$
\eta^{*}(\lambda)=\frac{\eta(\lambda)}{\eta_{c}}, \quad f(\lambda) \equiv \frac{G}{G_{0}}=\exp \left[m\left(\frac{1}{\lambda}-1\right)\right] .
$$

We notice here that the dimensionless quantity that governs the departure from the inelastic Generalized Newtonian behaviour is in fact

$$
W i(\lambda)=\frac{W i}{f(\lambda)},
$$

since $\operatorname{Wi}(\lambda) \rightarrow 0 \Rightarrow \boldsymbol{\tau}^{M *}=2 \eta^{*}(\lambda) \mathbf{D}^{*}$. In this sense, Wi( $\left.\lambda\right)$ can be identified as a local Weissenberg number that depends on the microstructure level.

The flow curve, given by Eq. (10) is made dimensionless as follows

$$
\tau^{*}=\zeta_{j}^{*}\left(\dot{\gamma}^{*}\right)\left[\tau_{y}^{*}+K^{*}\left(\dot{\gamma}^{*}\right)^{n}\right]+\eta_{\infty}^{*} \dot{\gamma}^{*},
$$

where

$$
\begin{aligned}
& \dot{\gamma}^{*}=\frac{L_{c} \dot{\gamma}}{U_{c}}, \quad \zeta_{1}^{*}\left(\dot{\gamma}^{*}\right)=1-\exp \left[-\frac{\eta_{0}^{*} \dot{\gamma}^{*}}{\tau_{\mathrm{y}}^{*}}\right], \quad \zeta_{2}^{*}\left(\dot{\gamma}^{*}\right)=\frac{\eta_{0}^{*} \dot{\gamma}^{*}}{\sqrt{\left(\eta_{0}^{*} \dot{\gamma}^{*}\right)^{2}+\tau_{\mathrm{y}}^{* 2}},} \quad \eta_{0}^{*}=\frac{\eta_{0}}{\eta_{\mathrm{c}}}, \quad \eta_{\infty}^{*}=\frac{\eta_{\infty}}{\eta_{\mathrm{c}}} . \\
& \zeta_{3}^{*}\left(\dot{\gamma}^{*}\right)= \begin{cases}1 & \text { if } \tau^{*} \geq \tau_{y}^{*} \\
\frac{\left(\eta_{0}^{*}-\eta_{\infty}^{*}\right) \dot{\gamma}^{*}}{\tau_{y}^{*}+K^{*} \dot{\gamma}^{* n}} & \text { if } \quad \tau^{*}<\tau_{y}^{*}\end{cases}
\end{aligned}
$$

In order to find the equilibrium structure parameter, we need now to use a dimensionless deviatoric part of the stress tensor, i.e.

$$
\sigma_{d e v}^{*}=\frac{\sigma_{d e v}}{\tau_{c}}
$$

and the Newton iterative process can be performed accordingly. The rest of the procedure is straightforward till the calculation of $\lambda_{\text {eq }}^{*}$ given by

$$
\lambda_{e q}^{*}=\frac{\ln \eta_{e q}^{*}-\ln \eta_{\infty}^{*}}{\ln \eta_{0}^{*}-\ln \eta_{\infty}^{*}}
$$


After that, we are able to feed the dimensionless evolution equation for the structure parameter, obtained from Eq. (18), given by

$$
\frac{\partial \lambda^{*}}{\partial t^{*}}+\nabla^{*} \cdot\left(\mathbf{u}^{*} \lambda^{*}\right)=\frac{1}{t_{e q}^{*}}\left(1-\frac{\lambda^{*}}{\lambda_{e q}^{*}}\right)
$$

where

$$
t_{e q}^{*}=t_{e q} \frac{U_{c}}{L_{c}}, \quad t^{*}=t \frac{U_{c}}{L_{c}} .
$$

In Cartesian coordinates, the boundary conditions given by Eqs. (26) and (27) can be written in dimensionless form, respectively, as

$$
\begin{aligned}
& -p^{*}+\frac{2 \eta_{\infty}^{*}}{\operatorname{Re}}\left[\frac{\partial u^{*}}{\partial x^{*}} n_{x}^{2}+\frac{\partial v^{*}}{\partial y^{*}} n_{y}^{2}+\left(\frac{\partial u^{*}}{\partial y^{*}}+\frac{\partial v^{*}}{\partial x^{*}}\right) n_{x} n_{y}\right]+\frac{1}{R e}\left[\left(\tau_{x x}^{M}\right)^{*} n_{x}^{2}+2\left(\tau_{x y}^{M}\right)^{*} n_{x} n_{y}+\left(\tau_{y y}^{M}\right)^{*} n_{y}^{2}\right]=F_{n}^{*}, \\
& \frac{\eta_{\infty}^{*}}{\operatorname{Re}}\left[2 \frac{\partial u^{*}}{\partial x^{*}} n_{x} m_{x}+\frac{\partial v^{*}}{\partial y^{*}} n_{y} m_{y}+\left[\frac{\partial u^{*}}{\partial y^{*}}+\frac{\partial v^{*}}{\partial x^{*}}\right]\left(n_{y} m_{x}+n_{x} m_{y}\right)\right]+\frac{1}{\operatorname{Re}}\left[\left(\left(\tau_{x x}^{M}\right)^{*}-\left(\tau_{y y}^{M}\right)^{*}\right) n_{x} n_{y}+\left(\tau_{x y}^{M}\right)^{*}\left(n_{y}^{2}-n_{x}^{2}\right)\right]=F_{m}^{*} .
\end{aligned}
$$

\section{Numerical formulation}

The numerical method used for solving the elasto-viscoplastic-thixotropic model is based in a Marker-And-Cell-type formulation (Harlow \& Welch, 1965). In the context of finite differences, we have modified our implicit scheme developed for free surface viscoelastic fluid flows (Oishi, Martins, Tomé, Cuminato, \& McKee, 2011). A detailed description of the numerical formulation used in this work will be addressed in this Section. From now on the dimensionless equations will be presented without the asterisk symbol, $*$.

\subsection{Temporal discretization}

Firstly, we note that the conservation Eqs. (32) and (33) can be discretized in the time as

$$
\begin{aligned}
& \nabla \cdot \mathbf{u}^{(n+1)}=0 \\
& \frac{\mathbf{u}^{(n+1)}}{\delta t}-\frac{\eta_{\infty}}{2 \operatorname{Re}} \nabla^{2} \mathbf{u}^{(n+1)}=\frac{\mathbf{u}^{(n)}}{\delta t}+\frac{\eta_{\infty}}{2 \operatorname{Re}} \nabla^{2} \mathbf{u}^{(n)}-\frac{3}{2} \nabla \cdot(\mathbf{u u})^{(n)}+\frac{1}{2} \nabla \cdot(\mathbf{u u})^{(n-1)}-\nabla p^{(n+1)}+\frac{1}{\operatorname{Re}} \nabla \cdot\left(\boldsymbol{\tau}^{M}\right)^{\left(n+\frac{1}{2}\right)}+\frac{1}{F r^{2}} \mathbf{g} .
\end{aligned}
$$

In Eq. (49), the diffusive terms are discretized by the Crank-Nicolson scheme while the convective terms are approximated by the Adams-Bashforth method.

The constitutive Eq. (35) is solved by the second order accuracy Runge-Kutta scheme (RK21), i.e. the Maxwell-like tensor, $\boldsymbol{\tau}^{M}$, is computed by

$$
\frac{\left(\boldsymbol{\tau}^{M}\right)^{(n+1)}-\left(\overline{\boldsymbol{\tau}}^{M}\right)^{(n+1)}}{\delta t}=\frac{1}{2}\left[\mathbf{F}_{\mathbf{1}}\left(\mathbf{u}, \boldsymbol{\tau}^{M}, \lambda\right)+\mathbf{F}_{2}\left(\mathbf{u}, \boldsymbol{\tau}^{M}, \lambda\right)\right]
$$

where

$$
\mathbf{F}_{\mathbf{1}}\left(\mathbf{u}, \boldsymbol{\tau}^{M}, \lambda\right)=\mathbf{F}\left(\mathbf{u}^{(n)},\left(\boldsymbol{\tau}^{M}\right)^{(n)}, \bar{\lambda}^{(n+1)}\right)
$$

and

$$
\mathbf{F}_{2}\left(\mathbf{u}, \boldsymbol{\tau}^{M}, \lambda\right)=\mathbf{F}\left(\mathbf{u}^{(n+1)},\left(\overline{\boldsymbol{\tau}}^{M}\right)^{(n+1)}, \lambda^{(n+1)}\right)
$$

with

$$
\mathbf{F}\left(\mathbf{u}, \boldsymbol{\tau}^{M}, \lambda\right)=-\nabla \cdot\left(\mathbf{u} \boldsymbol{\tau}^{M}\right)+\left[(\nabla \mathbf{u}) \cdot \boldsymbol{\tau}^{M}+\boldsymbol{\tau}^{M} \cdot(\nabla \mathbf{u})^{\mathbf{T}}\right]+\frac{f(\lambda)}{W i}\left[-\frac{1}{\eta(\lambda)} \boldsymbol{\tau}^{M}+2 \mathbf{D}\right] .
$$

The intermediate value of $\left(\overline{\boldsymbol{\tau}}^{M}\right)^{(n+1)}$ is obtained by the explicit forward Euler discretization:

$$
\frac{\left(\overline{\boldsymbol{\tau}}^{M}\right)^{(n+1)}-\left(\boldsymbol{\tau}^{M}\right)^{(n)}}{\delta t}=\mathbf{F}_{\mathbf{1}}\left(\mathbf{u}^{(n)},\left(\boldsymbol{\tau}^{M}\right)^{(n)}, \bar{\lambda}^{(n+1)}\right) .
$$

Notice that from Eq. (54) the divergent of $\boldsymbol{\tau}^{M}$ in Eq. (49) can be estimated by

$$
\nabla \cdot\left(\boldsymbol{\tau}^{M}\right)^{\left(n+\frac{1}{2}\right)}=\frac{1}{2}\left[\nabla \cdot\left(\boldsymbol{\tau}^{M}\right)^{(n)}+\nabla \cdot\left(\overline{\boldsymbol{\tau}}^{M}\right)^{(n+1)}\right] .
$$


One important feature of the elasto-viscoplastic thixotropic model is that the viscosity and the elastic modulus are considered to be functions of the structural parameter. Therefore, the values of $\eta(\lambda)$ and $f(\lambda)$ in Eq. (53) are computed from Eq. (37) considering the structure parameter at an intermediate time-level $\bar{\lambda}^{(n+1)}$ and at the final time-level $\lambda^{(n+1)}$. In order to enhance the stability of the transient computations, the structure parameter Eq. (44) is solved by a semi-implicit scheme using the following strategy:

$$
\left(\frac{1}{\delta t}+\frac{1}{t_{e q} \lambda_{e q}}\right) \Xi=\frac{\Theta_{1}}{\delta t}+\frac{1}{t_{e q}}-\nabla \cdot\left(\Theta_{2}\right),
$$

where $\Xi=\bar{\lambda}^{(n+1)}, \Theta_{1}=\lambda^{(n)}$ and $\Theta_{2}=\mathbf{u}^{(n)} \lambda^{(n)}$ or $\Xi=\lambda^{(n+1)}, \Theta_{1}=\bar{\lambda}^{(n+1)}$ and $\Theta_{2}=\mathbf{u}^{(n+1)} \bar{\lambda}^{(n+1)}$ according to the stage of the algorithm (see more details in Section 3.6).

\subsection{Numerical computation of the equilibrium structure parameter}

One of the essential features of a time-dependent model is the fact that is common to have non-equilibrium states in the domain. These states are characterized by a relation between stress and rate of strain which do not belong to the flow curve. In order to obtain the equilibrium point, i.e. the point of the flow curve corresponding to the current non-equilibrium state, we assume that the current stress value is the equilibrium stress value. Therefore, the corresponding equilibrium rate of strain value, $\dot{\gamma}_{e q}$, is obtained from Eq. (39) substituting $\tau$ by $\sigma_{\text {dev }}$ and $\dot{\gamma}$ by $\dot{\gamma}_{e q}$, which (dropping the asterisk) can be written as

$$
\sigma_{d e v}=\zeta_{j}\left(\dot{\gamma}_{e q}\right)\left[\tau_{y}+K\left(\dot{\gamma}_{e q}\right)^{n}\right]+\eta_{\infty} \dot{\gamma}_{e q}
$$

where $\sigma_{\text {dev }}$ is obtained from Eqs. (19) and (42).

In order to calculate the numerical value of $\lambda_{e q}$, firstly, $\dot{\gamma}_{e q}$ is obtained from solving Eq. (57) by an iterative procedure combining the bisection step and the Newton's method.

Once calculated the equilibrium deformation rate, the equilibrium viscosity is obtained from Eq. (20). After this, Eq. (43) is used to compute the solution of $\lambda_{e q}$.

To access $\lambda_{e q}$, which is used in Eq. (56) when $\Xi=\bar{\lambda}^{(n+1)}$, the value of $\sigma_{\text {dev }}$ in Eq. (57) is available considering the temporal discretization of the total tensor $\sigma$ (see Eq. (6)) at the time-level $(n)$. The solution of $\lambda_{\text {eq }}$ to be used in Eq. (56) for $\Xi=\lambda^{(n+1)}$ follows a similar procedure; however, in this stage of the algorithm, $\sigma_{\text {dev }}$ is constructed from Eq. (6) considering the final velocity field $\mathbf{u}^{(n+1)}$ and the intermediate value of the non-Newtonian part of the stress tensor $\left(\overline{\boldsymbol{\tau}}^{M}\right)^{(n+1)}($ see Eq. (54)). In the case of the bi-viscosity model, when $\sigma_{\text {dev }}<\tau_{y}, \lambda_{\text {eq }}=1$. In Section 3.6, the algorithm used in this work will be summarized.

\subsection{Decoupling of velocity and pressure fields: conservation equations of mass and momentum}

The numerical solution of Eqs. (48) and (49) involves a coupling of velocity and pressure fields since these variables are discretized in the same time level $(n+1)$. In this work, a pressure-segregation method known as projection method (Chorin, 1968; Temam, 1969) is applied in these equations for decoupling velocity and the pressure. The projection method which is mathematically rooted on the Helmholtz-Hodge theorem (Chorin \& Marsden, 2000) employs the decomposition of a vector field into gradient and solenoidal components, i.e.

$$
\widetilde{\mathbf{u}}^{(n+1)}=\mathbf{u}^{(n+1)}+\nabla \psi^{(n+1)},
$$

where $\psi$ is a potential function.

In summary, in the context of the projection method, Eq. (49) is solved for the intermediate value of the velocity $\widetilde{\mathbf{u}}^{(n+1)}$ with an approximated value of the pressure filed, denoted as $p^{(n)}$, i.e.,

$$
\frac{\widetilde{\mathbf{u}}^{(n+1)}}{\delta t}-\frac{\eta_{\infty}}{2 \operatorname{Re}} \nabla^{2} \widetilde{\mathbf{u}}^{(n+1)}=\frac{\mathbf{u}^{(n)}}{\delta t}+\frac{\eta_{\infty}}{2 \operatorname{Re}} \nabla^{2} \mathbf{u}^{(n)}-\frac{3}{2} \nabla \cdot(\mathbf{u u})^{(n)}+\frac{1}{2} \nabla \cdot(\mathbf{u u})^{(n-1)}-\nabla p^{(n)}+\nabla \cdot\left(\boldsymbol{\tau}^{M}\right)^{\left(n+\frac{1}{2}\right)}+\frac{1}{F r^{2}} \mathbf{g} .
$$

Taking the divergence of Eq. (58) and imposing mass conservation on $\mathbf{u}^{(n+1)}$, we obtain a Poisson equation for the potential $\psi$

$$
\nabla^{2} \psi^{(n+1)}=\nabla \cdot \widetilde{\mathbf{u}}^{(n+1)}
$$

The boundary conditions for solving the Poisson Eq. (60) will be discussed in the next Section.

Once obtained in the first sub-step of the projection method the intermediate velocity $\widetilde{\mathbf{u}}^{(n+1)}$ and pressure $\psi^{(n+1)}$, the second sub-step is applied: compute the final velocity and pressure fields from the following equations

$$
\begin{aligned}
\mathbf{u}^{(n+1)} & =\widetilde{\mathbf{u}}^{(n+1)}-\nabla \psi^{(n+1)} \\
p^{(n+1)} & =p^{(n)}+\frac{\psi^{(n+1)}}{\delta t}-\frac{\eta_{\infty}}{2 \operatorname{Re}} \nabla^{2} \psi^{(n+1)} .
\end{aligned}
$$


Details about the construction of projection methods (or fractional step methods) can be found in Guermond, Minev, and Shen (2006).

\subsection{Implicit strategy for the normal stress boundary condition}

In this work two types of boundary conditions are imposed for solving the Poisson Eq. (60). In rigid walls, where the no-slip condition for the velocity field is imposed to solve Eq. (49), traditional homogeneous Neumann conditions $\frac{\partial \psi}{\partial n}=0$ are used in the solution of Poisson Eq. (60). On the other hand, when the normal stress boundary condition (or traction boundary condition) Eq. (46) is enforced in some part of the domain, modified boundary conditions for $\psi$ in the solution of Eq. (60) are constructed in order to improve the stability of the numerical method for low Reynolds number flows. This strategy was first proposed by Oishi, Tomé, Cuminato, and McKee (2008) to the simulation of three-dimensional viscoelastic free surface flows. In that paper, the authors showed that the normal boundary condition (26) on the free surface needs to be treated implicitly to overcome a parabolic type stability condition. A similar formulation was presented in Poux, Glockner, and Azaiez (2012) for the case of open or traction boundary conditions in confined channel flows creating a new approach to enforce boundary conditions in the solution of the Poisson Eq. (60).

In this present paper, the method proposed in Oishi et al. (2011) will be adapted for simulating the elasto-viscoplastic thixotropic fluid flows in the presence of the normal stress boundary condition. Initially, Eq. (46) is discretized in time-level $(n+1)$ yielding

$$
\begin{gathered}
-p^{(n+1)}+\frac{2 \eta_{\infty}}{\operatorname{Re}}\left[\frac{\partial u^{(n+1)}}{\partial x} n_{x}^{2}+\frac{\partial v^{(n+1)}}{\partial y} n_{y}^{2}+\left(\frac{\partial u^{(n+1)}}{\partial y}+\frac{\partial v^{(n+1)}}{\partial x}\right) n_{x} n_{y}\right] \\
+\frac{1}{\operatorname{Re}}\left[\left(\tau_{x x}^{M}\right)^{(n+1)} n_{x}^{2}+2\left(\tau_{x y}^{M}\right)^{(n+1)} n_{x} n_{y}+\left(\tau_{y y}^{M}\right)^{(n+1)} n_{y}^{2}\right]=F_{n} .
\end{gathered}
$$

Notice that the equality $\frac{\partial u^{(n+1)}}{\partial x}=-\frac{\partial v^{(n+1)}}{\partial y}$ which is obtained from Eq. (48) can be incorporated into Eq. (63), i.e.,

$$
\begin{aligned}
& -p^{(n+1)}+\frac{2 \eta_{\infty}}{\operatorname{Re}}\left[-\frac{\partial v^{(n+1)}}{\partial y} n_{x}^{2}-\frac{\partial u^{(n+1)}}{\partial x} n_{y}^{2}+\left(\frac{\partial u^{(n+1)}}{\partial y}+\frac{\partial v^{(n+1)}}{\partial x}\right) n_{x} n_{y}\right] \\
& +\frac{1}{\operatorname{Re}}\left[\left(\tau_{x x}^{M}\right)^{(n+1)} n_{x}^{2}+2\left(\tau_{x y}^{M}\right)^{(n+1)} n_{x} n_{y}+\left(\tau_{y y}^{M}\right)^{(n+1)} n_{y}^{2}\right]=F_{n} .
\end{aligned}
$$

In order to construct a segregated solution, firstly, the pressure field needs to be decoupled from the velocity field in Eq. (64). This may be accomplished following the idea of the projection method as was described in Section 3.3 for the conservation equations (48)and (49). In summary, Eqs. (61) and (62), which are used in the projection method for the update velocity and pressure fields, respectively, will be introduced in Eq. (64). Notice that the presence of the non-Newtonian tensor $\left(\boldsymbol{\tau}^{M}\right)^{(n+1)}$ in Eq. (64) maintains a coupling of this tensor with the velocity field since its value is obtained from the constitutive Eq. (50). Therefore, the next step for obtaining the segregated solution of Eq. (64) is to assume an approximation for the non-Newtonian tensor, i.e., $\left(\boldsymbol{\tau}^{M}\right)^{(n+1)} \approx\left(\overline{\boldsymbol{\tau}}^{M}\right)^{(n+1)}$. Combining the above information, one can verify that $(64)$ is rewritten as

$$
\begin{aligned}
& \frac{\psi^{(n+1)}}{\delta t}-\frac{2 \eta_{\infty}}{\operatorname{Re}}\left[\left(\frac{\partial^{2} \psi^{(n+1)}}{\partial y^{2}}\right) n_{x}^{2}+\left(\frac{\partial^{2} \psi^{(n+1)}}{\partial x^{2}}\right) n_{y}^{2}-2\left(\frac{\partial^{2} \psi^{(n+1)}}{\partial x \partial y}\right) n_{x} n_{y}\right]-\frac{\eta_{\infty}}{2 R e} \nabla^{2} \psi^{(n+1)} \\
& =\frac{2 \eta_{\infty}}{\operatorname{Re}}\left[-\left(\frac{\partial \tilde{v}^{(n+1)}}{\partial y}\right) n_{x}^{2}-\left(\frac{\partial \tilde{u}^{(n+1)}}{\partial x}\right) n_{y}^{2}+\left(\frac{\partial \tilde{u}^{(n+1)}}{\partial y}+\frac{\partial \tilde{v}^{(n+1)}}{\partial x}\right) n_{x} n_{y}\right] \\
& \quad+\frac{1}{\operatorname{Re}}\left[\left(\bar{\tau}_{x x}^{M}\right)^{(n+1)} n_{x}^{2}+2\left(\bar{\tau}_{x y}^{M}\right)^{(n+1)} n_{x} n_{y}+\left(\bar{\tau}_{y y}^{M}\right)^{(n+1)} n_{y}^{2}\right]-p^{(n)}-F_{n} .
\end{aligned}
$$

Therefore, Eq. (65) is used as a modified boundary condition for $\psi$ in the solution of the Poisson equation (60) when the normal stress boundary condition is enforced.

\subsection{Basic ideas of the marker-and-cell method}

In this work the spatial discretization is performed with a MAC scheme in which velocities (in $x$ - and $y$-direction) are stored at the cell edges (positions $\left(i+\frac{1}{2}, j\right)$ and $\left(i, j+\frac{1}{2}\right)$, respectively) while the pressure, the non-Newtonian part of the stress tensor and the structure parameter are stored at cell centers (positions $(i, j)$ ).

A fundamental point to note about the MAC method is the classification of the grid cells according to their position relative to the fluid. In summary, the following classification is adopted:

- Empty Cell [E]: Cells that do not contain fluid;

- Full Cell [F]: Cells that do not have any face in contact with empty cells;

- Surface Cell [S]: Cells that contain fluid and have one or more faces in contact with empty cells;

- Boundary Cell [B]: Cells that belong to rigid boundaries; 
- Inflow Cell [I]: Cells that simulate fluid entrance into the domain;

- Outflow Cell [O]: Cells that define fluid exits of the domain.

This classification of cells needs to be updated at each time step as the fluid moves in the domain.

Another important feature of the MAC method is the strategy about marker particles dynamics. The MAC method which is one of the most famous methods for the numerical simulation of free surface flows combines the front-tracking method (see Tryggvason, Scardovelli, \& Zaleski, 2011) with an Eulerian framework for representing the free surface. In summary, in this strategy, virtual marker particles are distributed into each cell of an Eulerian grid and the interface is recovered by interpolation with piecewise continuous functions. During the course of one time step the displacement of the marker particles is accomplished by solving the equation

$$
\dot{\mathbf{x}}=\mathbf{u} \text {. }
$$

Details of a comprehensive review of the MAC methodology can be found in McKee et al. (2008) and more recently in Cuminato, Oishi, and Figueiredo (2014).

\subsection{The solution algorithm}

In the context of a segregated formulation, an overview of the solution algorithm is now given. The algorithm used in the present paper follows the improved version of the MAC-type algorithm proposed by Oishi et al. (2011) (see also (Martins, Oishi, Afonso, \& Alves, 2015)). In particular, new steps are incorporated for solving the complex rheological model analyzed in this work which depends of the time-dependent microstructure.

Assuming that at time $t=t_{n}$ the total tensor $\sigma$, the structure parameter $\lambda$ and the markers positions $\mathbf{x}$ are known, the MAC algorithm for transient motions of elasto-viscoplastic thixotropic materials can be summarized as:

- Step 1 - Calculate $\bar{\lambda}^{(n+1)}$ from Eq. (56). In this stage, the value of the equilibrium parameter $\lambda_{\text {eq }}$ at level time $(n)$ is obtained according to the procedure described in Section 3.2.

- Step 2 - Calculate $\left(\overline{\boldsymbol{\tau}}^{M}\right)^{(n+1)}$ from Eq. (54).

- Step 3 - Calculate the intermediate velocity $\widetilde{\mathbf{u}}^{(n+1)}$ from Eq. (59) using the Adams-Bashforth/Crank-Nicolson scheme.

- Step 4 - Solve the Poisson Equation (60) assuming homogeneous Neumann conditions and modified boundary conditions for $\psi$ as presented in Section 3.4 .

- Step 5 - Update the velocity field from Eq. (61);

- Step 6 - Update the pressure field from Eq. (62);

- Step 7 - Calculate $\lambda_{e q}^{(n+1)}$ following the methodology presented in Section 3.2 and update the structure parameter $\lambda^{(n+1)}$ using Eq. (56).

- Step 8 - Calculate $\left(\boldsymbol{\tau}^{M}\right)^{(n+1)}$ using Eq. (50);

Remark: For free surface flows, the last step of the algorithm is to update the positions of the marker particles by solving the ODE's Eq. (66).

\section{Problems description}

\subsection{Start-up in a plane channel}

In a first problem, we consider a flow between two fixed parallel plates $\left(-L_{c} \leq y \leq L_{c}\right)$ with length $10 L_{c}$.

\subsubsection{Boundary conditions}

- At the inlet, an entrance pressure, $p_{i n}$ is imposed. This condition is expressed by

$$
\begin{aligned}
& \mathbf{n}_{i n} \cdot \boldsymbol{\sigma}_{i n} \cdot \mathbf{n}_{i n}^{\mathrm{T}}=-p_{i n}, \\
& \mathbf{m}_{i n} \cdot \boldsymbol{\sigma}_{i n} \cdot \mathbf{n}_{i n}^{\mathrm{T}}=0,
\end{aligned}
$$

where $\mathbf{n}_{i n}=\left(n_{x}, n_{y}\right)$ and $\mathbf{m}_{i n}=\left(m_{x}, m_{y}\right)$ are the normal and tangential vectors at the inlet, respectively, and $\boldsymbol{\sigma}_{i n}$ is the stress tensor at the inlet.

- At the outlet, the pressure, $p_{\text {out }}$, is also imposed. Hence,

$$
\begin{aligned}
& \mathbf{n}_{\text {out }} \cdot \boldsymbol{\sigma}_{\text {out }} \cdot \mathbf{n}_{\text {out }}^{\mathrm{T}}=-p_{\text {out }}, \\
& \mathbf{m}_{\text {out }} \cdot \boldsymbol{\sigma}_{\text {out }} \cdot \mathbf{n}_{\text {out }}^{\mathrm{T}}=0,
\end{aligned}
$$

where $\mathbf{n}_{\text {out }}=\left(n_{x}, n_{y}\right)$ and $\mathbf{m}_{\text {out }}=\left(m_{x}, m_{y}\right)$ are the normal and tangential vectors at the outlet, respectively, and $\boldsymbol{\sigma}_{\text {out }}$ is the stress tensor at the outlet.

- At rigid walls, no-slip and impermeability boundary condition are imposed for the velocity field. For the structure parameter, $\lambda$, the Neumann condition is employed while for the non-Newtonian part, $\boldsymbol{\tau}^{M}$, the values were linearly extrapolated to the rigid wall cells. 


\subsubsection{Initial conditions}

- zero flow field for velocity

$$
\mathbf{u}\left(x, y, t_{0}\right)=\mathbf{0} .
$$

- fully structured condition for the structure parameter,

$$
\lambda\left(x, y, t_{0}\right)=1 \text {. }
$$

- pressure field obeying constant pressure gradient condition,

$$
p\left(x, y, t_{0}\right)=p_{\text {in }}-\frac{p_{\text {in }}-p_{\text {out }}}{10 L_{c}} x
$$

- stress field equilibrating the pressure field,

$$
\tau_{y x}^{M}\left(x, y, t_{0}\right)=\frac{p_{\text {in }}-p_{\text {out }}}{10 L_{c}} y
$$

\subsection{Rejuvenation of a square block under the action of gravity}

The second problem analyzed is a square block made of an elasto-viscoplastic material which is initially at rest and fully structured. We assume that the block has been kept confined for a time long enough for the pressure field to equilibrate the gravitational forces. Since the stress due to pressure is isotropic, at this stage no destruction occurs. Suddenly, at time $t=t_{0}$, the pressure along the interface is set to the atmospheric pressure. This non-equilibrium initial condition leads to a stress rearrangement that can cause a destruction of the material.

\subsubsection{Boundary conditions}

- At the bottom rigid wall, no-slip and impermeability boundary condition are imposed for velocity field $(\mathbf{u}=\mathbf{0})$. In the case of the structure parameter, a Neumann condition is applied with a zero gradient in the direction of the bottom plane. The non-Newtonian part of the stress tensor, $\boldsymbol{\tau}^{M}$, is linearly extrapolated to the cell boundary.

- The other boundaries are delimited by a free surface. In the case where surface tension is negligible, these boundary conditions are represented by

$$
\begin{aligned}
& \mathbf{n} \cdot \boldsymbol{\sigma} \cdot \mathbf{n}^{\mathrm{T}}=-p_{a t m}, \\
& \mathbf{m} \cdot \boldsymbol{\sigma} \cdot \mathbf{n}^{\mathrm{T}}=0,
\end{aligned}
$$

where $\mathbf{n}$ and $\mathbf{m}$ are the normal and tangential vectors at the interface, respectively, and $p_{a t m}$ is the atmospheric pressure. Without loss of generality, we consider $p_{a t m}=0$.

\subsubsection{Initial conditions}

When the material is fully structured and at rest the only force acting is due to gravity. The pressure distribution needed to equilibrate gravity, assuming that $p\left(x\left(t_{0}\right), H\left(t_{0}\right)\right)=0$, is given by

$$
p\left(x, y, t_{0}\right)=\rho g\left[H\left(t_{0}\right)-y\right],
$$

for $x$ and $y$ in the interior of the domain. In Eq. (77), $H\left(t_{0}\right)$ is the initial height of the square block.

\section{Numerical results for the plane channel}

The numerical methodology described in Section 3 is now applied in the simulation of the flow of an elasto-viscoplastic thixotropic material in a plane channel with dimensions $\left[-5 L_{c}, 5 L_{c}\right] \times\left[-L_{c}, L_{c}\right]$. We fixed the characteristic stress and length scales as $\tau_{c}=50 \mathrm{~Pa}$ and $L_{c}=0.5 \mathrm{~m}$. Due to the implicit nature of Eq. (29), we fixed the characteristic velocity as $U_{c}=$ $0.0707 \mathrm{~m} / \mathrm{s}$ and, therefore, the dimensional parameter $\eta_{\infty}$ is determined a posteriori, i.e. when the dimensionless yield stress and dimensionless consistency index are set. In the present work we considered a Bigham material i.e. a null consistency index. Having defined $\tau_{c}, U_{c}$, and $L_{c}$, the characteristic viscosity can be calculated, and in this case is $\eta_{c}=318.51$ Pa $\mathrm{s}$. The relationship of fully structured and unstructured viscosities is maintained fixed as $\eta_{0} / \eta_{\infty}=10^{5}$ and the pressure values in Eqs. (67) and (69) are also fixed as $p_{i n}=500 \mathrm{~Pa}$ and $p_{\text {out }}=0$, respectively. From now on the parameters used in all numerical simulations will be presented in dimensionless form. For all numerical tests, we consider the Reynolds number $R e=0.1$ while the parameter $m$ in Eq. (9) is adopted as $m=0.1$. We have tested different possibilities for $\zeta_{j}(\dot{\gamma})$. We have obtained negligible differences between these choices, but $\zeta_{3}(\dot{\gamma})$ performed with a much lower cost and was chosen for this reason. 


\subsection{Convergence results}

Firstly, we employ a mesh independence analysis in order to choose a final mesh where the results will be obtained. Since there is no analytical solution for the plane channel flow of the elasto-viscoplastic material of the present work, we use the results obtained from a very refined mesh, M3 $(800 \times 160)$-cells $\left(\delta x / L_{c}=\delta y / L_{c}=0.0125\right)$, as a reference solution. Two other meshes: M1 $(200 \times 40)$-cells $\left(\delta x / L_{c}=\delta y / L_{c}=0.05\right)$ and M2 $(400 \times 80)$-cells $\left(\delta x / L_{c}=\delta y / L_{c}=0.025\right)$ are compared to the reference solution. The plane channel flow was simulated on the above mentioned meshes until $t=100$. At this time the results did not show any change indicating that steady state had been achieved. The time-step used in the simulations was $\delta t=5 \times 10^{-4}$.

The convergence of the numerical method is exemplified in Fig. 1 where we present the solution profiles of the velocity $u$, the structure parameter $\lambda$ and the shear component of the non-Newtonian tensor, $\tau_{x y}^{M}$, obtained by the numerical method using $W i=0.1$ and $t_{e q}=10$ for some values of dimensionless yield stress: $\tau_{y}=0.25, \tau_{y}=0.5$ and $\tau_{y}=0.75$. Results are captured at time $t=100$. We can observe that the solutions obtained using meshes M1 and M2 agree well with the reference solution, specially those related to $u$ and $\tau_{x y}^{M}$ profiles. The $u$ profiles corresponding to the yield stresses analyzed reveal a plug flow at the center and a parabolic profile near the wall. The critical (dimensionless) positions where the $u$ profile abandons the uniform value, $\left|y_{c}\right|$, can be interpreted as an yield point. We can infer that, at time $t=100$, the steady state velocity profiles were achieved, since we obtained the expected result that $\left|y_{c}\right|=\tau_{y}$, i.e. the dimensionless position of the yield surface coincides with the dimensionless yield stress. The structure profiles show that, near the center, the material is in the fully structured state. However, close to $\left|y_{c}\right|$, the structure decays, experiencing a dramatic break down process around the yield position. The profiles associated to $\tau_{x y}^{M}$ obtained were also the expected ones for the fully-developed flow. In this case, one expects a constant pressure gradient and a linear total shear stress profile. Since the Newtonian shear stress is proportional to the shear rate, which vanishes for $|y|<\left|y_{c}\right|$, in this region, $\tau_{x y}^{M}=\sigma_{x y}$. Besides that, $|y| \geq\left|y_{c}\right| \Rightarrow\left|\tau_{x y}^{M}\right|=\tau_{y}$, i.e. the non-Newtonian part of the shear stress is constant and the total shear stress is given by a Bingham relation.

\subsection{Thixotropic behavior}

In this Section, we investigate the thixotropic effects in the Poiseuille flow varying the thixotropic equilibrium time, $t_{\text {eq }}$, the fluid yield stress, $\tau_{y}$, and the Weissenberg number, Wi. Considering mesh M2, we have plotted the profiles of the structure parameter $\lambda$ along the vertical line $x=0.0$ at different times of the simulations.

Fig. 2 shows the trends of the time-dependent behavior of the thixotropy for two values of $t_{\text {eq }}$, for instance $t_{\text {eq }}=10$ and $t_{e q}=40$, together with the elasto-viscoplastic case with no thixotropy, $t_{e q}=0$. For this test, we kept fixed $\tau_{y}=0.5$ and $W i=0.1$. The results are displayed for the following values of dimensionless times of simulation: $t=1,2,5,100$. The initial condition for the structure parameter is $\lambda=1$ at all points of the domain, i.e. the material begins in a fully structured state. For the $t_{e q}=0$ case, green symbols, we found a unique curve for all times, indicating that the material has reached its final state instantly and elasticity did not affect significantly its response. For the other two values of $t_{e q}$ we found expected evolutions in time for the structure parameter, $\lambda$. At the very beginning, the material starts a break down process near the wall, where the stresses are higher. As time elapses, the structure parameter decreases with more intensity near the wall, but leaving the material intact near the symmetry plane, $y=0$. The difference between the evolutions corresponding to the materials with $t_{e q}=10$ and with $t_{e q}=40$ can be explained from the perspective of Eq. (44). Increasing $t_{e q}$ leads to a decrease in the structure rate. Since the materials are experiencing a break down process, it is expected that, for the same elapsed time, at the same position, a higher structure level for the higher $t_{e q}$ material, as illustrated in Fig. 2 . We can notice that for $t=100$ the two curves coincide with the non-thixotropic case, showing that a steady state has been achieved.

We have also analyzed the variation of $\tau_{y}$ in the transient computations for fixed values of $t_{e q}=10$ and $W i=0.1$, as shown in Fig. 3a. The non-thixotropic case is presented in Fig. 3b, as a reference. Again, different times are considered to plot the profiles of the structure parameter for $\tau_{y}=0.25$ and $\tau_{y}=0.75$. The obtained results were as expected, i.e. the structure level decreases near the wall and leaves the core intact. As time elapses, the structure near the wall decreases more and more until the achievement of a steady state value. The size of the core where the material remains fully structured in the entire process is a function of the yield stress. A higher yield stress means that a greater portion of the material remains fully structured, since a smaller portion of the domain is subjected to stresses higher than the yield stress. Fig. $3 \mathrm{~b}$ shows that the purely elasto-viscoplastic case does not change in time, since there are no thixotropic effects and elasticity is low due to the low value of Weissenberg number $(W i=0.1)$. The curves for $\tau_{y}=0.25$ and $\tau_{y}=0.75$ correspond to the final curves $(t=100)$ obtained in Fig. 3a, showing that steady-state was reached at this time. These curves show quite clearly the dramatic drop in the structure parameter at $y / L_{c}=\tau_{y}$.

Finally, the time-dependent behavior of the material is studied from the elastic point of view by plotting the structure parameter in Fig. 4 for different times for two values of the Weissenberg number, namely $W i=0.01$ and $W i=5$. Fig. 4 a shows the evolution in time with fixed $\tau_{y}=0.5$ and $t_{e q}=10$, while Fig. $4 \mathrm{~b}$ displays the non-thixotropic case. According to these results, we can observe that the influence of the elasticity is relatively small specially at low times. At higher values of dimensionless time, we notice that the high Weissenberg number case is less structured. This happens due to the action of more intense normal stresses in this case. The $t_{e q}=0$ case shows elastic effects in the evolution in time for the highest Weissenberg number case, $W i=5$. The blue curves in Fig. $4 \mathrm{~b}$ clearly show a fading memory process. 

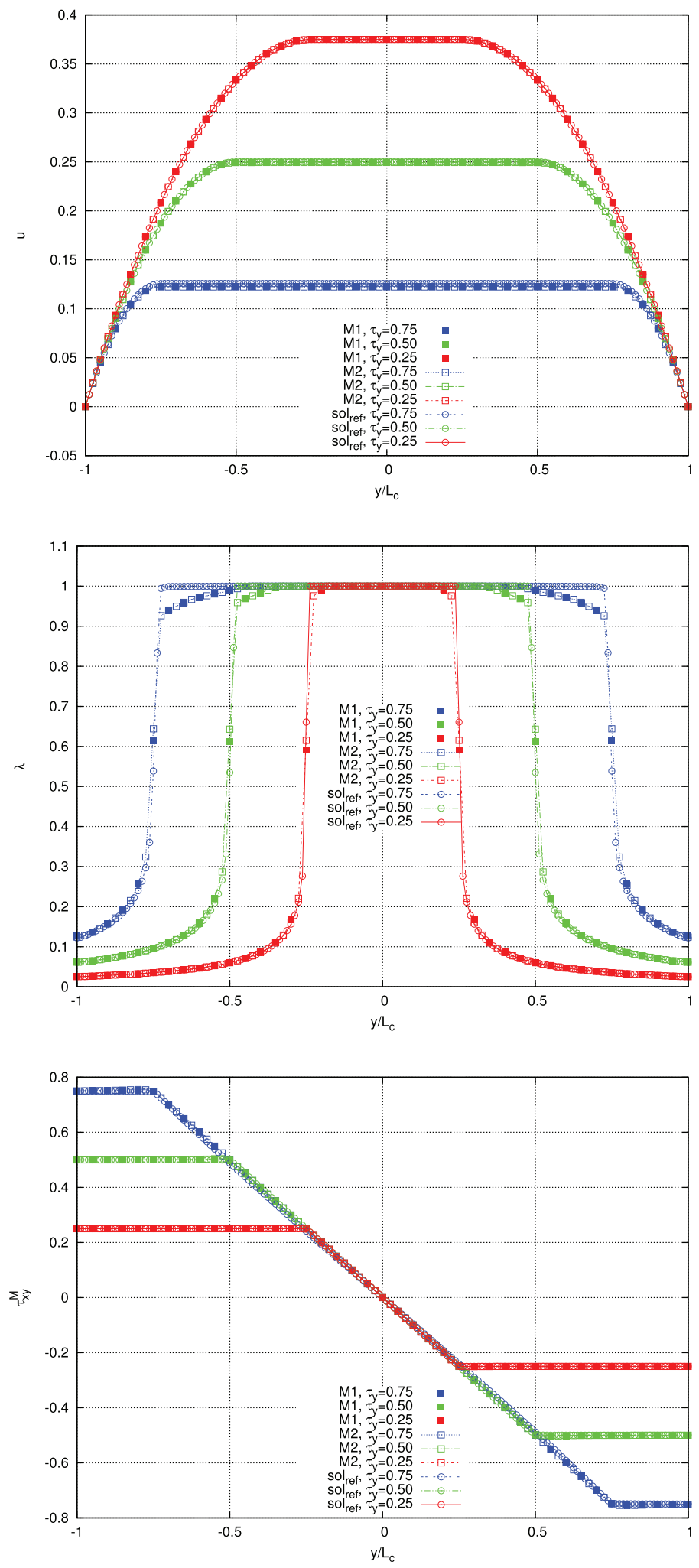

Fig. 1. Results for the profiles of the velocity $u$, the structure parameter $\lambda$ and the shear component of the non-Newtonian tensor $\tau_{x y}^{M}$ using $W i=0.1$ and $t_{e q}=10$ for different values of yield stress: $\tau_{y}=0.25, \tau_{y}=0.5$ and $\tau_{y}=0.75$, obtained on meshes M1, M2 and M3 (sol $l_{\text {ref }}$ ). 


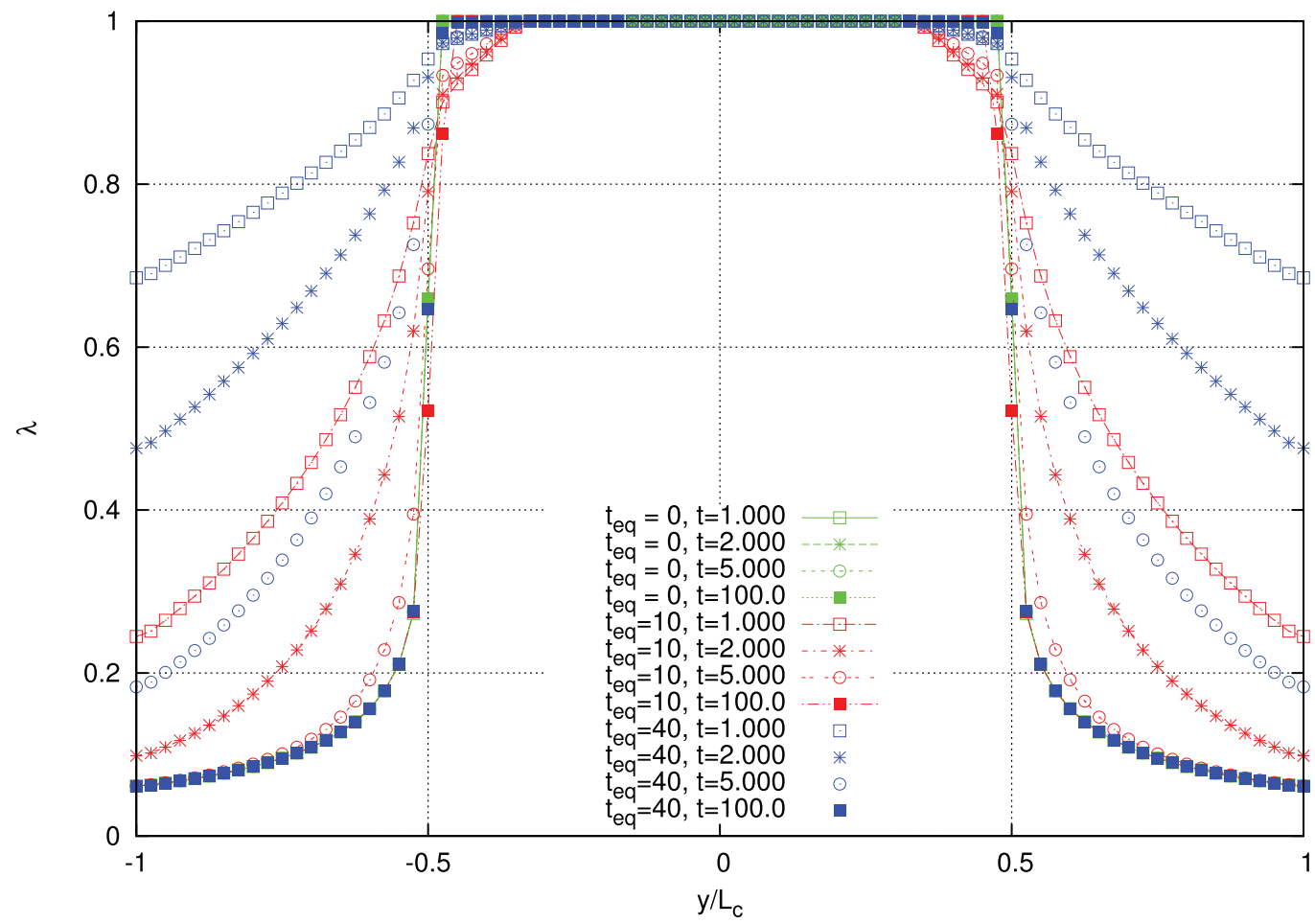

Fig. 2. Results for the profile of the structure parameter $\lambda$ along the vertical line $x=0.0$ for $W i=0.1$ and $\tau_{y}=0.5$ at different times $(t=1,2,5,100)$. Three values of the equilibrium time were used: $t_{e q}=0, t_{e q}=10$ and $t_{e q}=40$.

a)

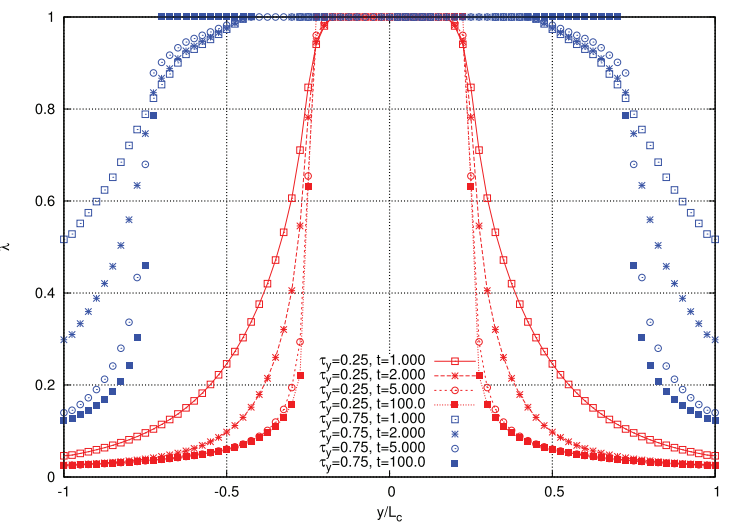

b)

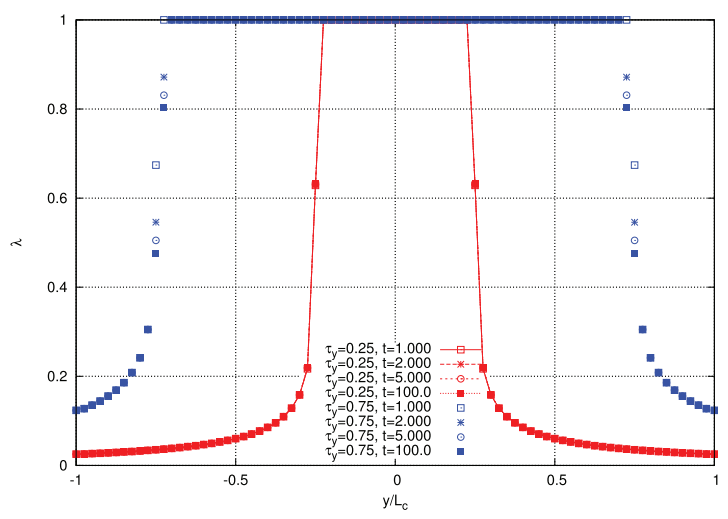

Fig. 3. Results for the profile of the structure parameter $\lambda$ along the vertical line $x=0.0$ for $W i=0.1$ and two values of the yields stress $\left(\tau_{y}=0.25\right.$ and $\left.\tau_{y}=0.75\right)$ at different times $(t=1,2,5,100):$ a) $t_{e q}=10$ and b) $t_{e q}=0$.

\subsection{Effects of the local Weissenberg number}

Fig. 5 shows an example of the time evolution of the local value of the Weissenberg number, $W i(\lambda)=\tau_{c} / G(\lambda)$, for the fixed value of nominal Weissenberg number, $W i=1$. The dimensionless yield stress and the thixotropic equilibrium time considered were $\tau_{y}=0.5$ and $t_{e q}=10$, respectively. As explained above, since $W i(\lambda)$ appears in the constitutive equation and this quantity is structure dependent, its value can be significantly different from the nominal one, depending on the dynamics of the problem. From Eqs. (37) and (38) we can see that $W i(\lambda) \leq W i$. Since in the core of the domain the stress intensity is below the yield stress, in this region the local Weissenberg number remains equal to the nominal one during the whole evolution. As time elapses $W i(\lambda)$ decreases maintaining its minimum value at the wall. In other words, near the wall, elastic forces are less predominant and its importance increases as we approximate the plug zone near the center. At time $t=100$ the problem is in its steady-state regime. We notice that at $t=5$ steady-state was already achieved near the wall. Other graphs for $W i(\lambda)$ corresponding to different values of $W i$ were very similar and are not shown here. 
a)

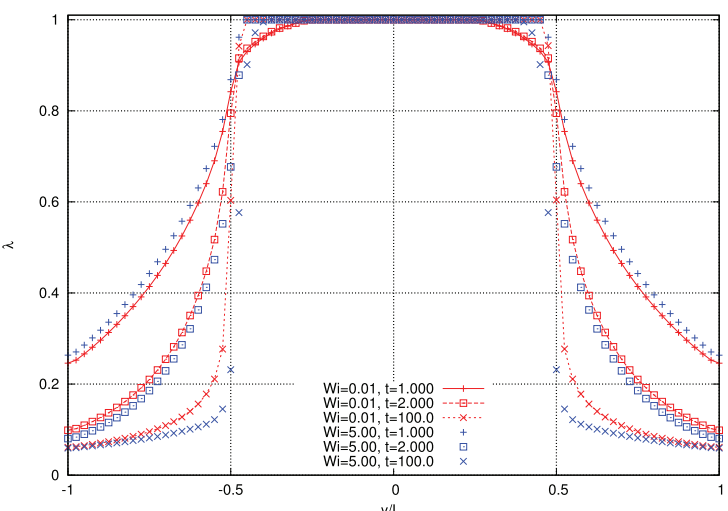

b)

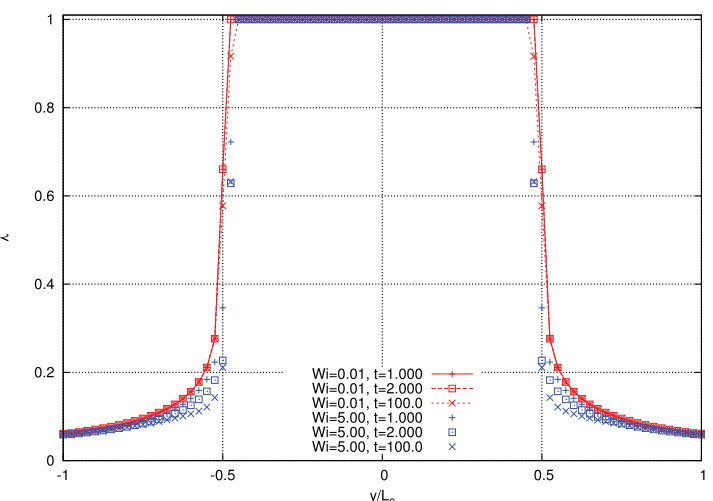

$y / L_{c}$

Fig. 4. Results for the profile of the structure parameter $\lambda$ along the vertical line $x=0.0$ for $\tau_{y}=0.5$ and two values of the Weissenberg number $(W i=0.01$ and $W i=5)$ at different times $(t=1,2,100):$ a) $t_{e q}=10$ and b) $t_{e q}=0$.

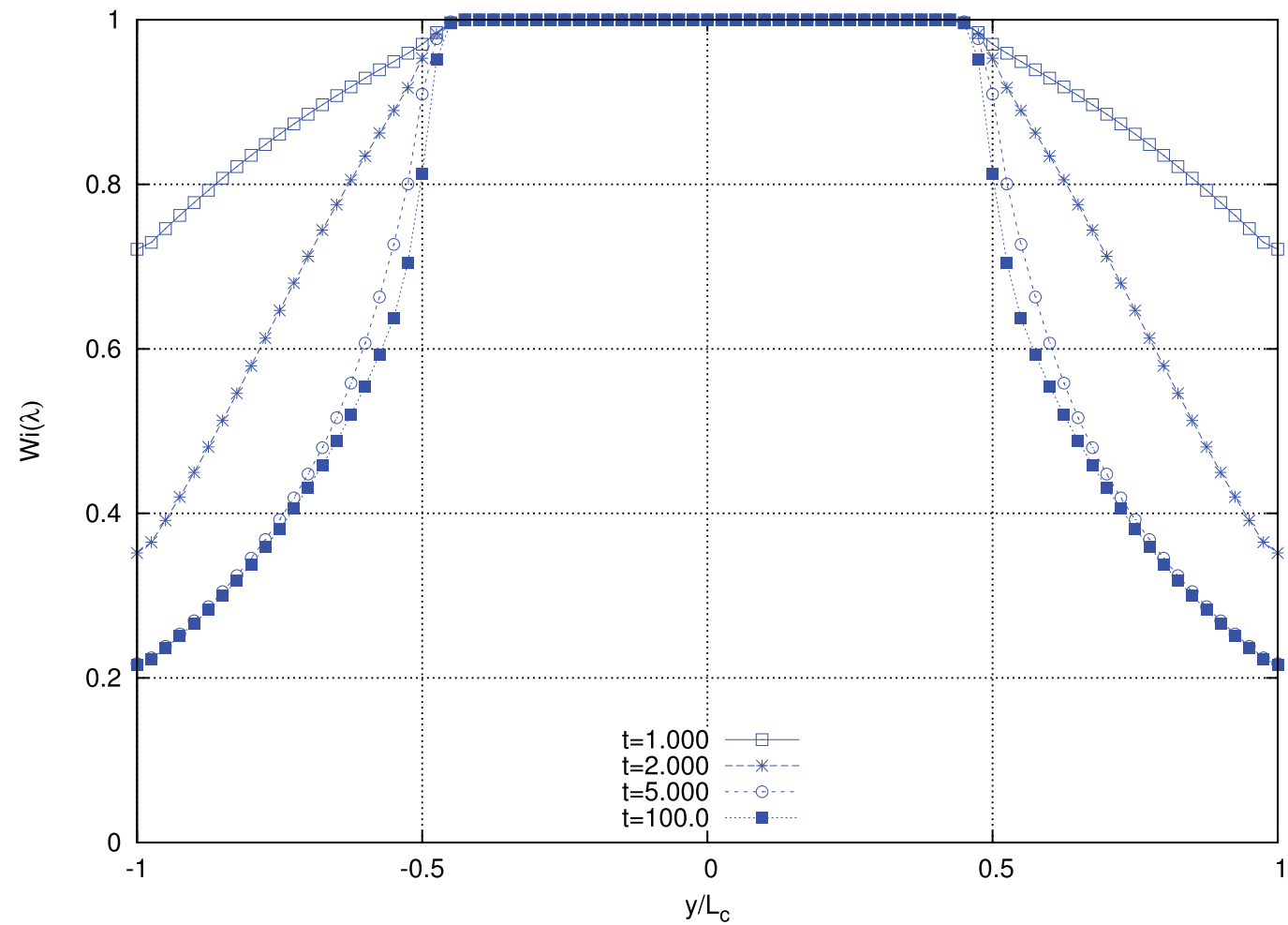

Fig. 5. Results for the profile of the the local value of the Weissenberg number, Wi( $\lambda$ ), along the vertical line $x=0.0$ for $\tau_{y}=0.5$ and $t_{e q}=10$ at different times $(t=1,2,5,100)$. The nominal Weissenberg number adopted is $W i=1$.

\section{A moving free surface problem: square shape of an EVPT material}

In order to assess the effect of thixotropy, elasticity and viscoplasticity in a moving free surface motion, we have simulated the problem described in Section 4. The initial height and length of the block are $0.2 \mathrm{~m}$ as shown in the Fig. $8 \mathrm{a}$ ). In this problem, the scaling parameters are $\tau_{c}=1132.7 \times 10^{3} \mathrm{~Pa}, \eta_{c}=673.13 \mathrm{~Pa} \mathrm{~s}, U_{c}=0.3365 \mathrm{~m} / \mathrm{s}$ and $L_{c}=0.2 \mathrm{~m}$. The fixed dimensionless numbers are $\operatorname{Re}=0.1$ and $\mathrm{Fr}=0.24$. Function $\zeta_{3}$ was again adopted and the parameter $m$ was also fixed at $m=0.1$. Surface tension is neglected in this problem. The time-step used in the simulations was $\delta t=10^{-5}$.

\subsection{Convergence results}

The numerical method has been verified with a mesh refinement using three meshes to define the material block: M1 $(40 \times 40)$-cells $\left(\delta x / L_{C}=\delta y / L_{C}=0.025\right), M 2(80 \times 80)$-cells $\left(\delta x / L_{C}=\delta y / L_{C}=0.0125\right)$ and $\mathrm{M} 3(160 \times 160)$-cells 
a)
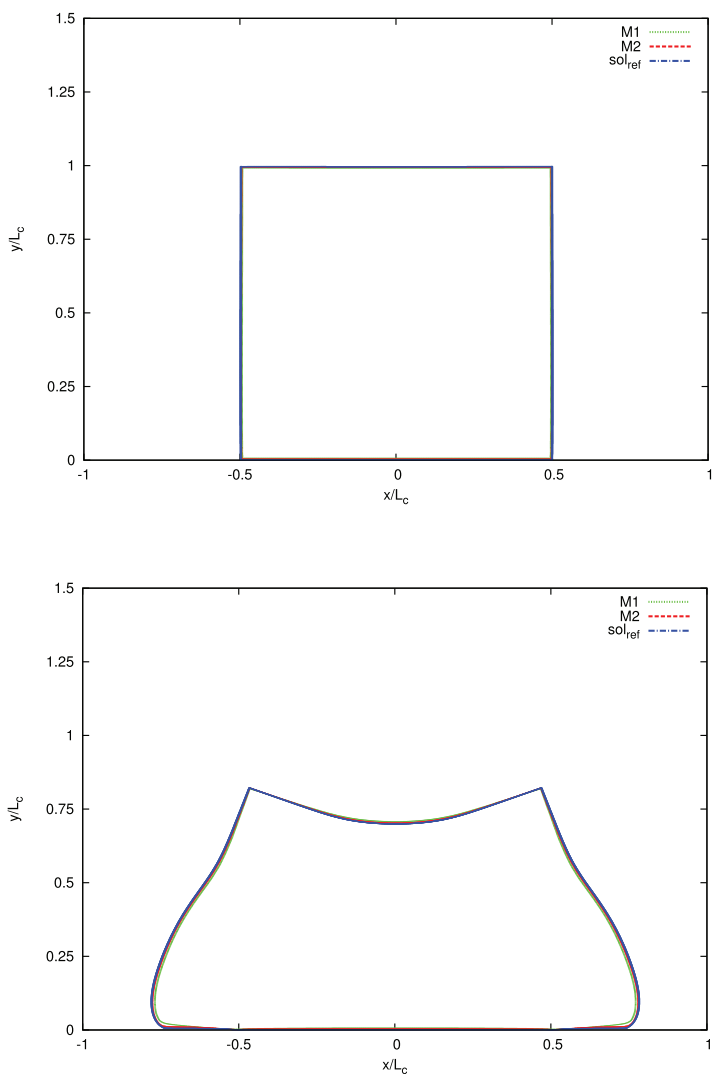

b)

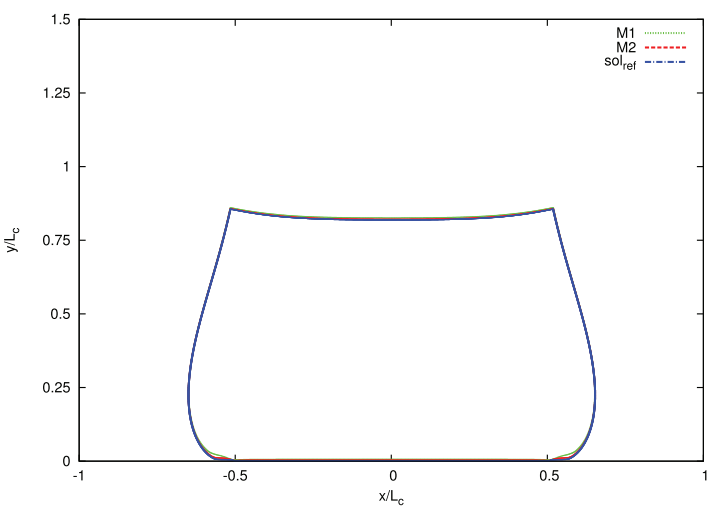

d)

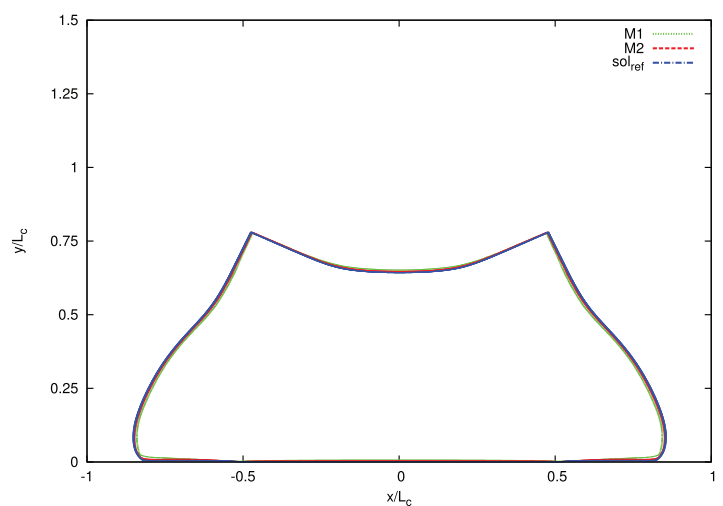

c)

Fig. 6. Free surface shape visualizations on meshes M1, M2 and M3 (sol $l_{\text {ref }}$ ) for $W i=0.01, \tau_{y}=0.3$ and $t_{e q}=100$ : a) $t=1$, b) $t=10$, c) $t=30$ and d) $t=50$.

$\left(\delta x / L_{c}=\delta y / L_{c}=0.00625\right)$. Simulations are performed with $W i=0.01$ and $W i=5$ for fixed values of $\tau_{y}=0.3$ and $t_{e q}=100$. The results of the free surface shape for $W i=0.01$ and $W i=5$ are given in Figs. 6 and 7, respectively, for different nondimensional times, for instance, $t U_{c} / L_{c}=1,10,30,50$. From these figures we can see a good agreement among the results considering the three meshes at different times. The influence of the variation of the Weissenberg number in the shape of the fluid block will be carefully discussed in the following Sections.

To supply further evidence concerning the convergence of the numerical method, we introduce a measure to monitor the horizontal distance $\left(\right.$ dist $\left._{x}(t)\right)$ between the leftmost and rightmost boundaries (horizontal spread of the fluid block) as a function of the time (see Fig. 8b)). Similarly, the vertical distance $\left(\right.$ dist $\left._{y}(t)\right)$ between the topmost and the bottommost is also monitored. At the initial time, $t_{0}$, the dimensionless distances are $\operatorname{dist}_{x}\left(t_{0}\right) / L_{c}=\operatorname{dist}_{y}\left(t_{0}\right) / L_{c}=H\left(t_{0}\right) / L_{c}=1$. Results for $\operatorname{dist}_{x}(t) / L_{c}$ considering $W i=0.01$ and $W i=5$ are presented in Fig. 9 using the three meshes adopted in this Section. In addition, Fig. 10 describes the results for $\operatorname{dist}_{y}(t) / L_{c}$. Again, the mesh refinement indicates the convergence of the results for the horizontal spread and vertical width of the fluid block.

\subsection{Influence of the model parameters}

In this Section we describe the influence of the elasto-viscoplastic thixotropic model parameters in the simulation of the free surface square block problem. The fixed dimensionless parameters for this study are $R e=0.1$ and $F r=0.24$ while mesh M2 is adopted in all simulations to reduce the CPU time. The main quantities whose changes are evaluated are the dimensionless yield stress, $\tau_{y}$, also called yield number; the Weissenberg number, Wi; and the dimensionless thixotropic equilibrium time, $t_{e q}$. These dimensionless parameters are associated to the level of yielding, elasticity, and thixotropy, respectively. There are three set of cases where one parameter is fixed while the other two are displayed in a Cartesian manner to illustrate the two-by-two combined effects.

\subsubsection{Combined effects of yield stress and Weissenberg number}

Firstly, we present a set of simulations to investigate the influence of $\tau_{y}$ and $W i$ on the structure parameter field at different times. In this set of simulations, we have fixed the thixotropic equilibrium time as $t_{e q}=250$. Figs. $11-15$ show the time evolution of the block shape and the structure parameter field at times $t=1,2,5,10$, and 20, respectively. 


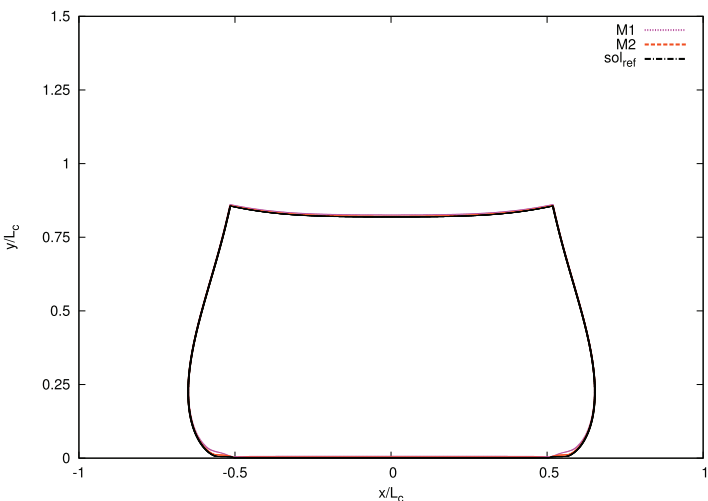

a)

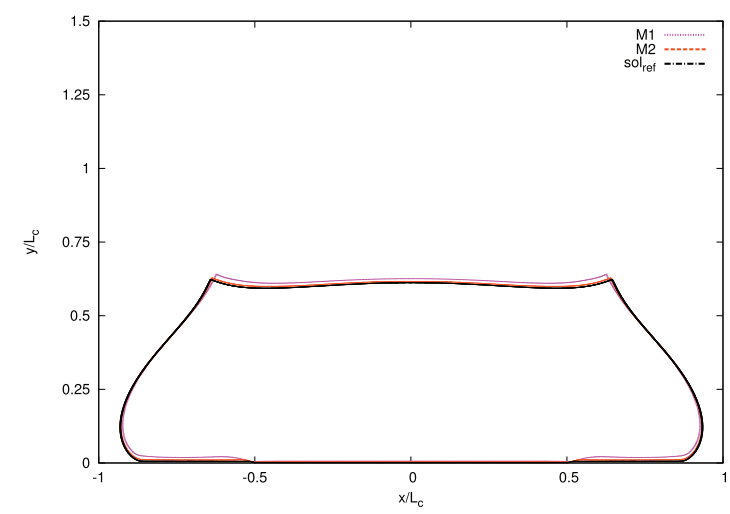

c)

d)

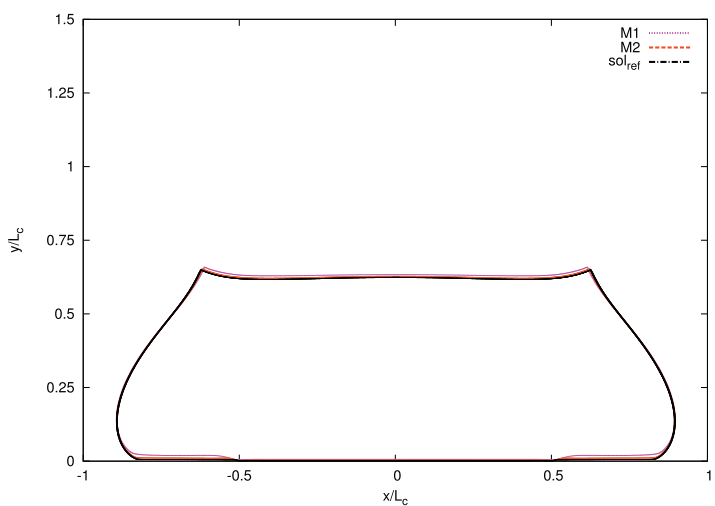

b)

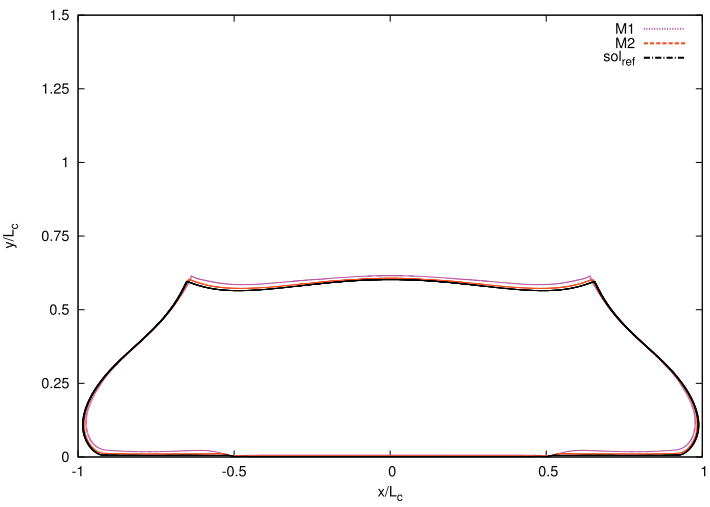

Fig. 7. Free surface shape visualizations on meshes M1, M2 and M3 ( $\left(\mathrm{sol}_{\text {ref }}\right)$ for $W i=5, \tau_{y}=0.3$ and $t_{\text {eq }}=100$ : a) $t=1$, b) $t=10$, c) $t=30$ and d) $t=50$.
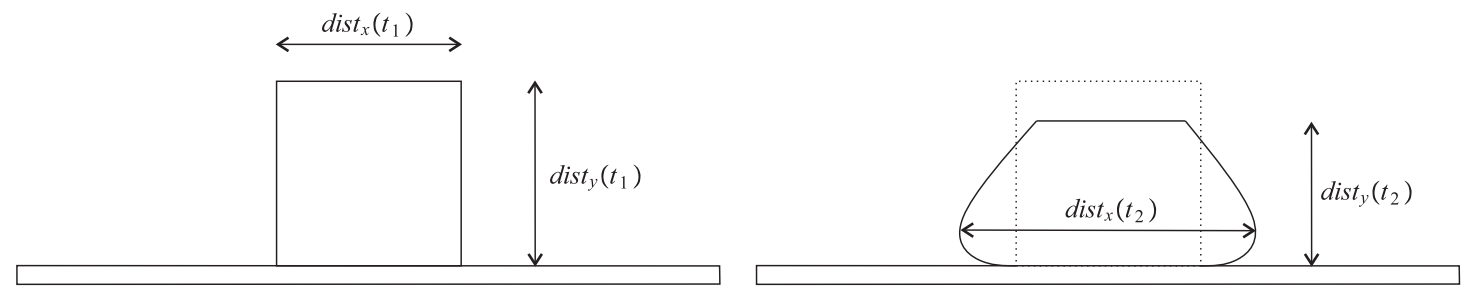

Fig. 8. Illustration of the the horizontal $\left(\operatorname{dist}_{x}(t)\right)$ and vertical $\left(d i s t_{y}(t)\right)$ distances for two times $t_{1}$ and $t_{2}$.

In the first time displayed, $t=1$, Fig. 11, we notice that the row at the top which corresponds to the lowest yield stress value $\left(\tau_{y}=0.1\right)$, presents the first signs of destruction. The regions at the bottom near the free surface present a more pronounced destruction although we can also find a decrease in the original structure level at the middle part of the block, away from the interface. The leftmost column, corresponding to a very low Weissenberg number $(W i=0.01)$, remains rigidly at the same initial shape. Changes with respect to the initial shape occur due to elastic effects mainly. At this stage, most of the gravity force is balanced by the elastic forces that are created by the block deformation. Since higher values of Weissenberg number correspond to a more flexible material, it is reasonable to expect that high Weissenberg number cases exhibit higher deformation in order to balance the same amount of force. Another worth noticing fact is that the yield number splits the stress into a plastic part and a viscous part, which are complementary with respect to unity, as shown by Eq. (30) (we notice here that we have employed this equation in the particular case where $\mathrm{K} *=0$ ). In other words, higher values of the dimensionless yield stress, imply lower values of the retardation dimensionless viscosity. Therefore, since in the present model elasticity is associated to a more solid-like response, an increase of the yield stress induces a more elastic behavior.

When time evolves to $t=2$, as shown in Fig. 12, we observe thixotropic effects taking place. The destruction continues mainly at the top row, although the second row, corresponding to a value of dimensionless yield stress of $\tau_{y}=0.3$, exhibits signs of destruction at the bottom. It is worth noticing that the decrease in structure level at the top row leaves an "island" of virgin material near the bottom. Although gravity forces are severe at this location, because of the symmetry at 


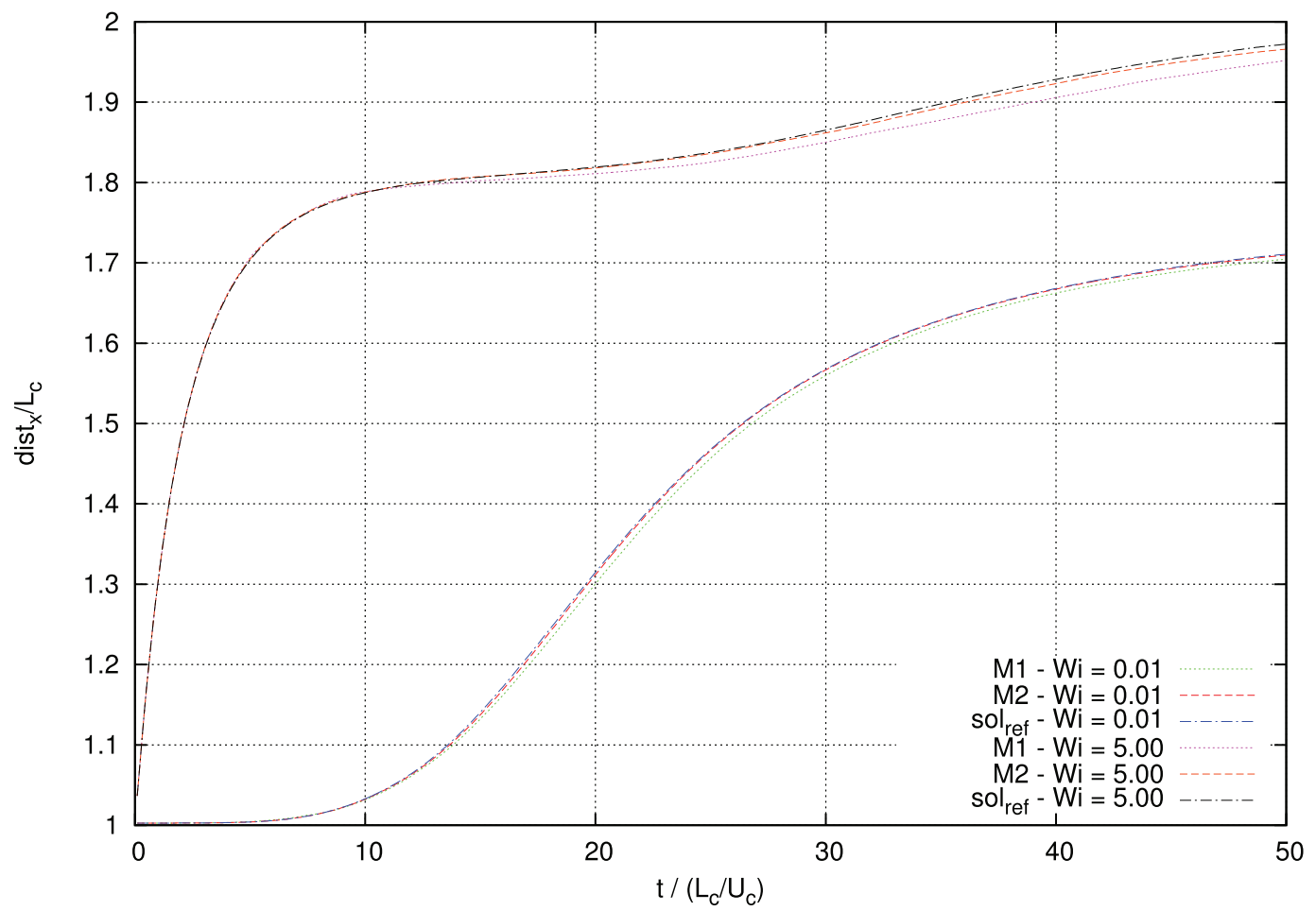

Fig. 9. Time evolution of the horizontal distance, $\operatorname{dist}_{x}(t) / L_{c}$, using meshes M1, M2 and M3 (sol $l_{\text {ref }}$ ) at fixed values of $\tau_{y}=0.3$ and $t_{e q}=100$.

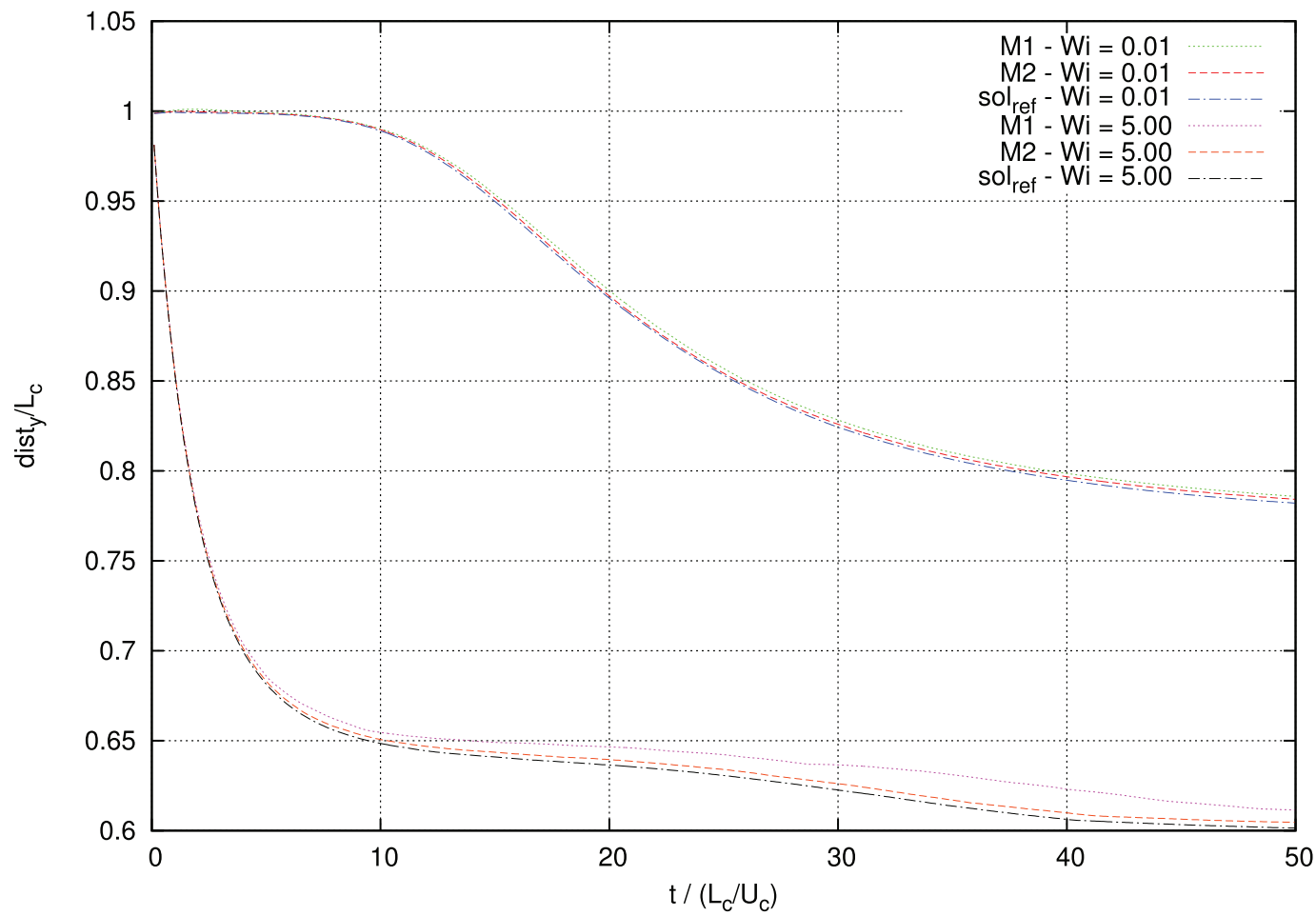

Fig. 10. Time evolution of the horizontal distance, dist $(t) / L_{c}$, using meshes M1, M2 and M3 (sol $\left.l_{\text {ref }}\right)$ at fixed values of $\tau_{y}=0.3$ and $t_{e q}=100$. 

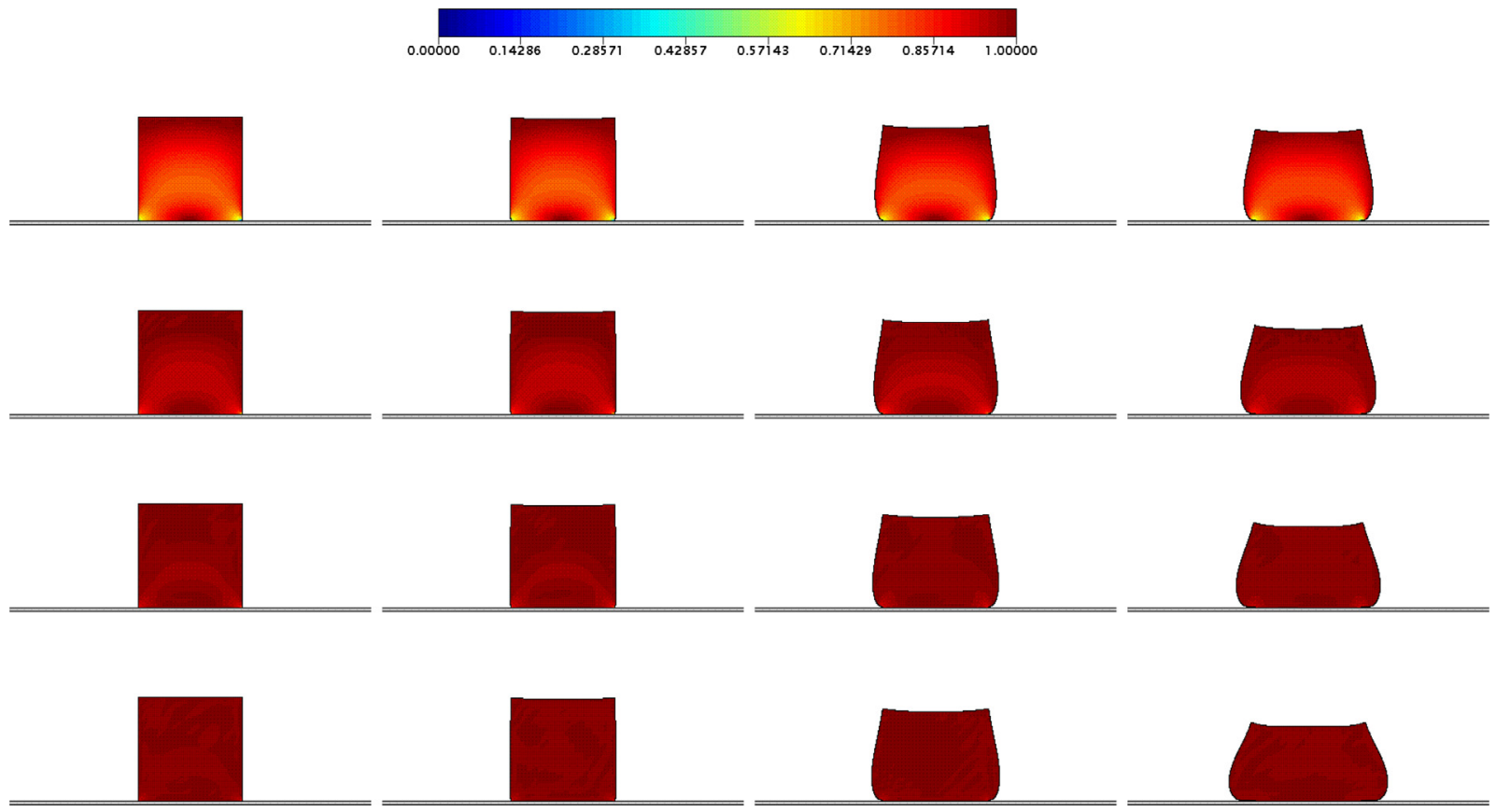

Fig. 11. Numerical prediction of the structure parameter $\lambda$ at the dimensionless time $t=1$. The equilibrium time adopted is $t_{e q}=250$. From (column) left to right: $W i=0.01,0.1,1,5$, and from (row) the top to bottom: $\tau_{y}=0.1,0.3,0.5,0.7$.

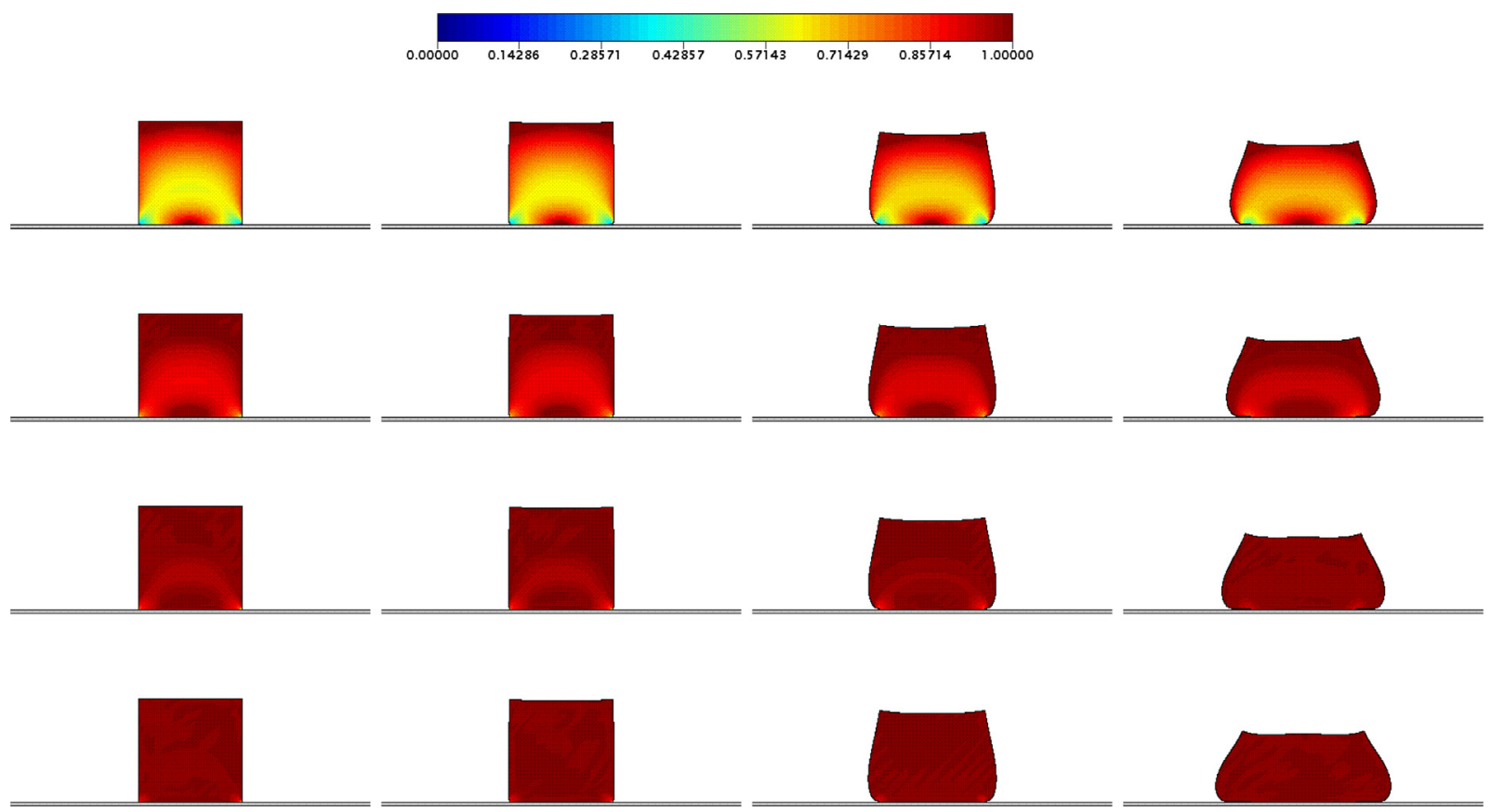

Fig. 12. Numerical prediction of the structure parameter $\lambda$ at the dimensionless time $t=2$. The equilibrium time adopted is $t_{e q}=250$. From (column) left to right: $W i=0.01,0.1,1,5$, and from (row) the top to bottom: $\tau_{y}=0.1,0.3,0.5,0.7$.

the vertical centerline, no anisotropic stress is generated and gravity is balanced by pressure. The leftmost column, corresponding to a very low Weissenberg number $(W i=0.01)$, continues rigidly at the same initial shape. On the other hand, the columns corresponding to higher Wi continue to evolve in shape, even for the cases where higher values of yield stress inhibit destruction. This fact shows that elastic effects are still responsible for this shape change.

At time $t=5$, Fig. 13, we can notice that other parts of the material in the top row have experienced a destruction process. A structure level of $\lambda \approx 0.5$ takes place in most of the domain. Very low structure levels appear at the bottom 


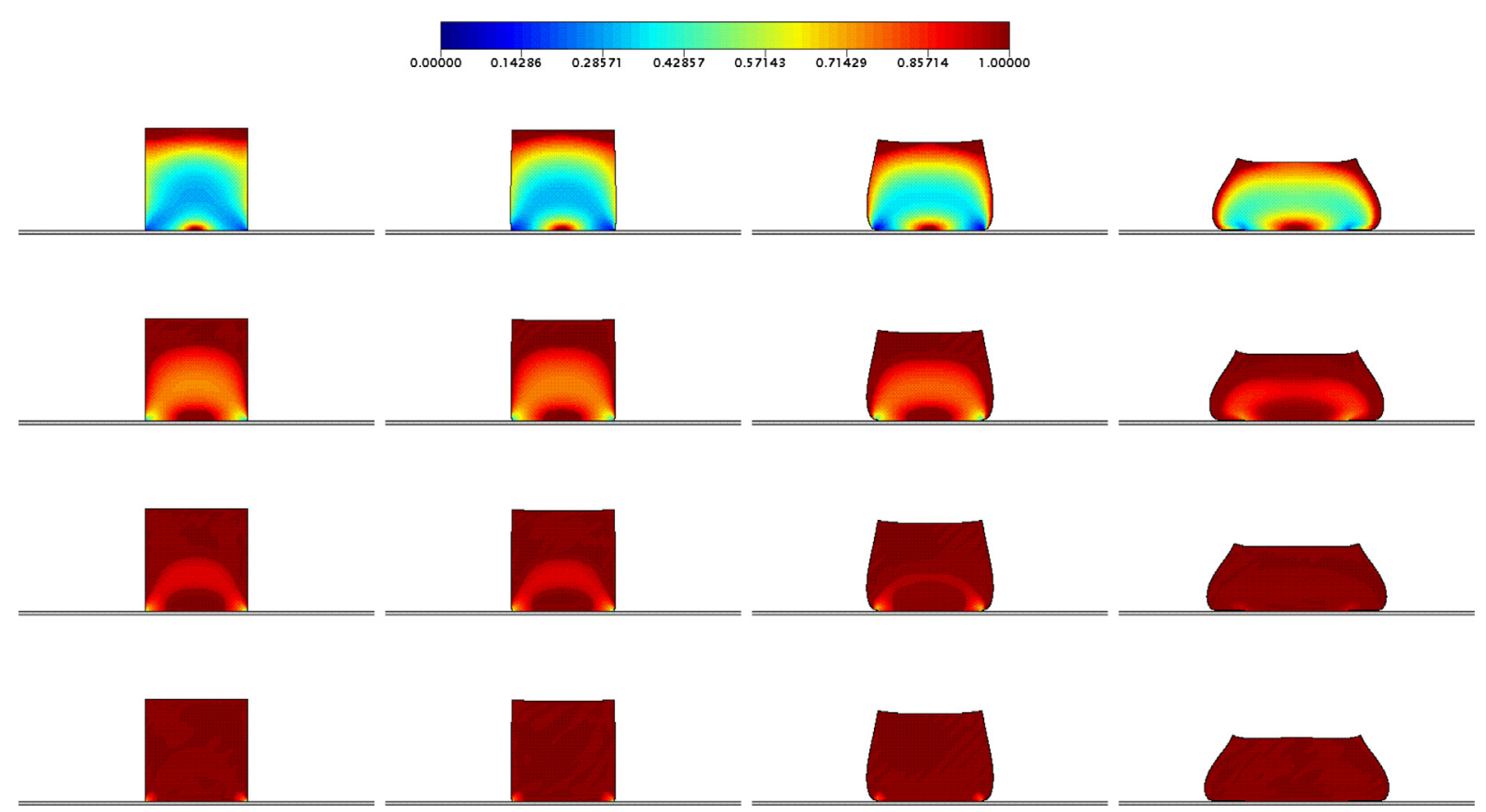

Fig. 13. Numerical prediction of the structure parameter $\lambda$ at the dimensionless time $t=5$. The equilibrium time adopted is $t_{e q}=250$. From (column) left to right: $W i=0.01,0.1,1,5$, and from (row) the top to bottom: $\tau_{y}=0.1,0.3,0.5,0.7$.

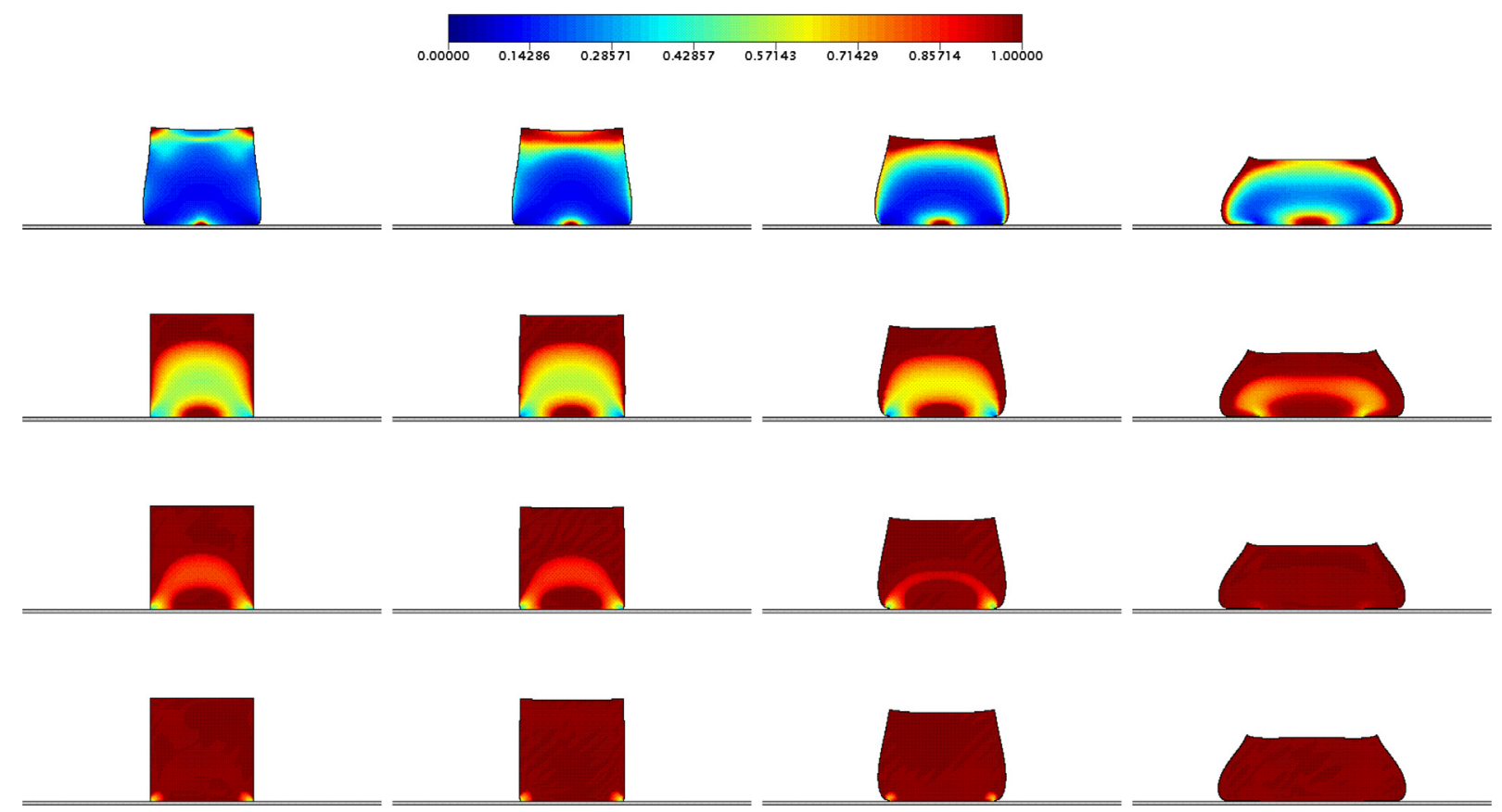

Fig. 14. Numerical prediction of the structure parameter $\lambda$ at the dimensionless time $t=10$. The equilibrium time adopted is $t_{e q}=250$. From (column) left to right: $W i=0.01,0.1,1,5$, and from (row) the top to bottom: $\tau_{y}=0.1,0.3,0.5,0.7$.

corners, except from the highest $W i$ case. We can notice a slight change in shape in the $\tau_{y}=0.1, W i=0.01$ case, showing a non-elastic effect. The fully-structured island at the bottom-center position starts to shrink from the sides, a sign of the presence of anisotropic stress at this location. At this time, the second row corresponding to a value of dimensionless yield stress of $\tau_{y}=0.3$ evolves toward more unstructured states, forming a similar virgin region at the bottom-center. In the $\tau_{y}=0.3, W i=5$ case we clearly notice that parts of the material which were not at the bottom occupies this region, what is perceived from a fully-structured state near bottom interface (this effect exists but is more timid in the 


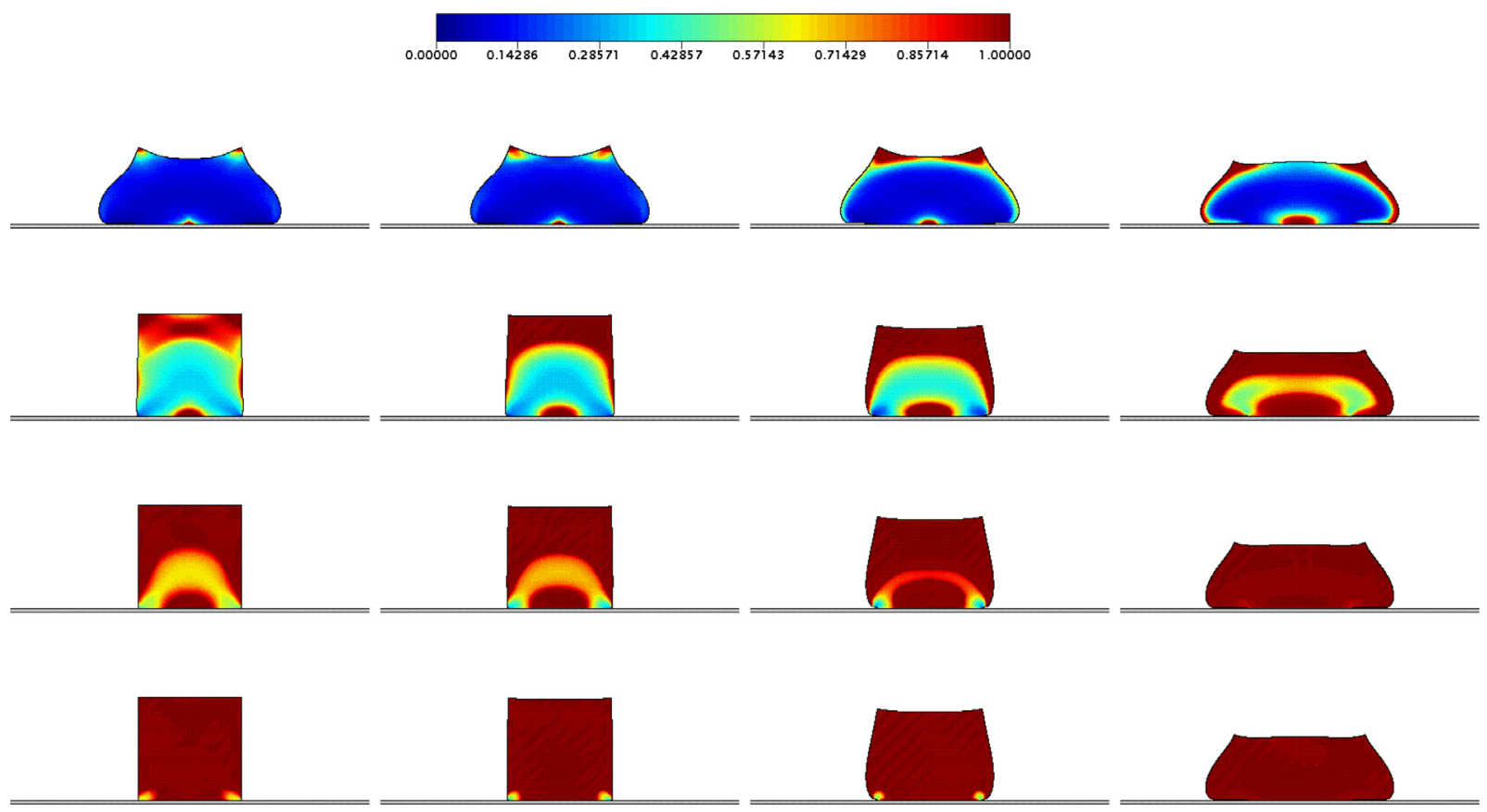

Fig. 15. Numerical prediction of the structure parameter $\lambda$ at the dimensionless time $t=20$. The equilibrium time adopted is $t_{e q}=250$. From (column) left to right: $W i=0.01,0.1,1,5$, and from (row) the top to bottom: $\tau_{y}=0.1,0.3,0.5,0.7$.

$\tau_{y}=0.1, W i=5$ case). At this time, the third row corresponding to $\tau_{y}=0.5$ begins a destruction process at the bottom corners. Again we reinforce that this is a thixotropic effect. The anisotropic stresses at these regions are beyond the yield stress from the beginning of the numerical experiment. However, because the difference between $\sigma_{d e v}$ and $\tau_{y}$ are smaller, the driving potential for destruction is lower and the thixotropic equilibrium time of $t_{e q}=250$ turns the structure change into a slower process.

Fig. 14 , corresponding to time $t=10$, shows an evolution of the same trends observed at time $t=5 \mathrm{~s}$. We can stress that $\tau_{y}=0.1, W i=0.01$ and $\tau_{y}=0.1, W i=0.1$ cases show a more accentuated non-elastic change in shape leading to the block a trapezoidal shape. The $\tau_{y}=0.5, W i=5$ and $\tau_{y}=0.7, W i=5$ cases show no structure decrease. Since their heights were decreased by elastic effects, the difference $\sigma_{\text {dev }}-\tau_{y}$ is low or negative even at the bottom.

In Fig. 15, that corresponds to time $t=20$, there is a more severe shape change of the top row cases, corresponding to $\tau_{y}=0.1$, which can be attributed to viscous effects. The great majority of the domain is characterized by a very low structure level and, as a consequence, the material is in a more mobile state. It is worth noticing that the more rigid cases of this row present a depression at the top-center region showing that the enlargement of the material is fed by dislocations at this region. This behavior contrasts with the $\tau_{y}=0.1, W i=5$ case, where a more uniform top dislocation occurs. When time advances to $t=50$, shown in Fig. 16, we notice a significant change in shape in the top row cases due to the flow of the material in a low structuring level, when the material is more mobile. Elasticity does not play an important role at this structuring level, as expected and perceived when we compare the different cases of the $\tau_{y}=0.1$ row. At this point in time, the second row, corresponding to $\tau_{y}=0.3$ shows a viscous change in shape more pronounced at low Weissenberg numbers. In this connection, we can observe that when $\tau_{y} \geq 0.3$ elasticity still plays an important role. For $\tau_{y}=0.5$ and $\tau_{y}=0.7$ no significant change in shape occurs, although a decrease in the structure level takes place. The evolution through times $t=100, t=150$, and $t=200$, as presented in Figs. 17, 18, and 19, respectively, shows aging in some regions. Since the stresses due to motion low down, their destruction action is not able to overcome the spontaneous build up process. From $t=50$ to $t=100$, we still can see a flow process with a shape change at low yield stress levels. From $t=150$ to $t=200$, there is no significant flow even at the top row.

\subsubsection{Combined effects of the thixotropic equilibrium time and the Weissenberg number}

The influence of the thixotropic equilibrium time and the Weissenberg number on the time evolution of the structure parameter on the material block is illustrated in Figs. 20-24 at selected dimensionless times. The yield stress used in these simulations is fixed at $\tau_{y}=0.3$.

As shown in the previous results, the firsts shape changes are due to elastic effects, i.e. increasing the Weissenberg number induces a more pronounced block deformation from its initial square shape. We can notice that from $t=1$ until $t=20$, Figs. 20-24, the material blocks in the leftmost column corresponding to the lowest Wi remain rigidly at the original configuration. On the other hand, in the rightmost column corresponding to the highest Wi the blocks show an evolution in 


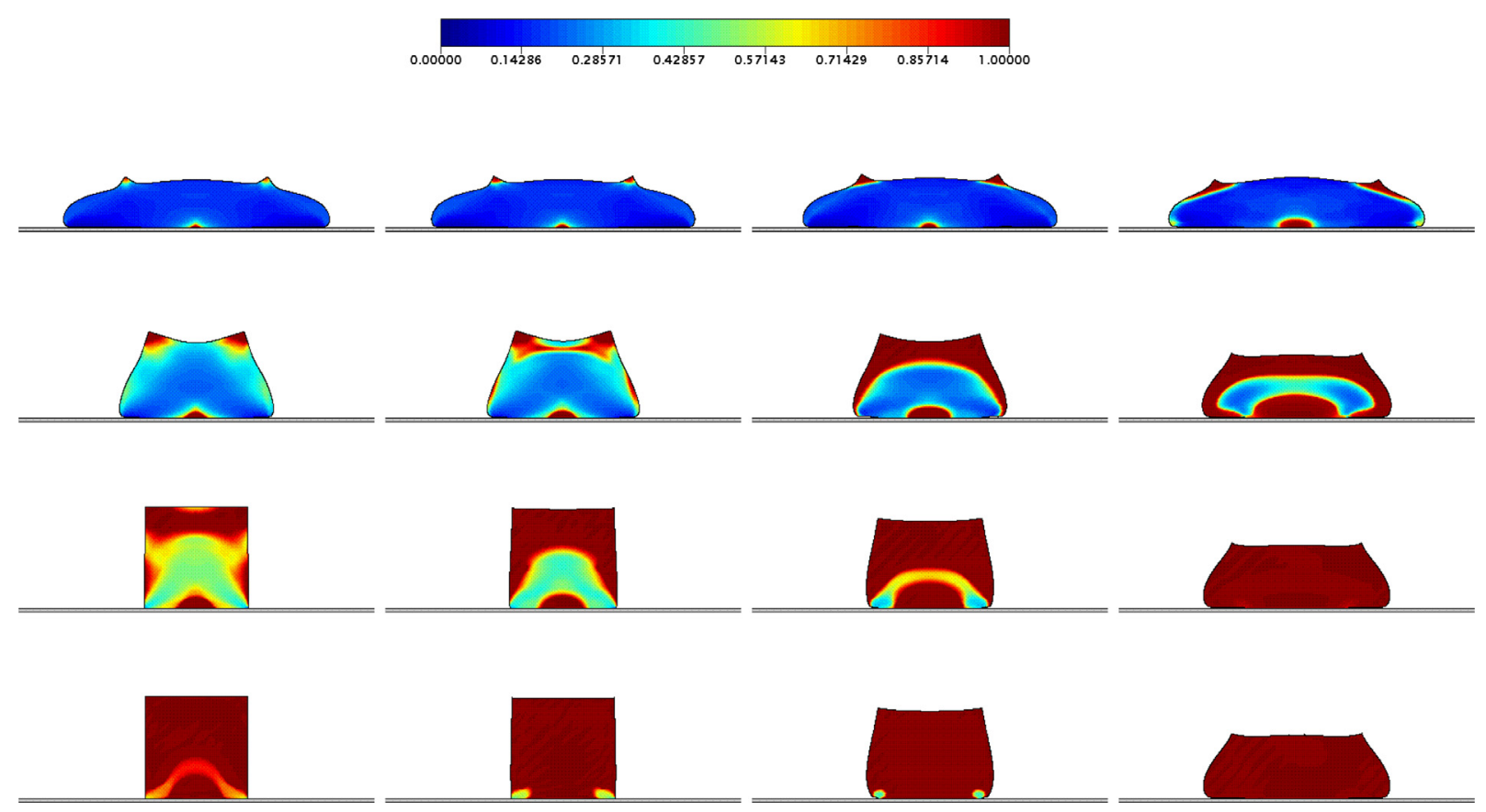

Fig. 16. Numerical prediction of the structure parameter $\lambda$ at the dimensionless time $t=50$. The equilibrium time adopted is $t_{e q}=250$. From (column) left to right: $W i=0.01,0.1,1,5$, and from (row) the top to bottom: $\tau_{y}=0.1,0.3,0.5,0.7$.

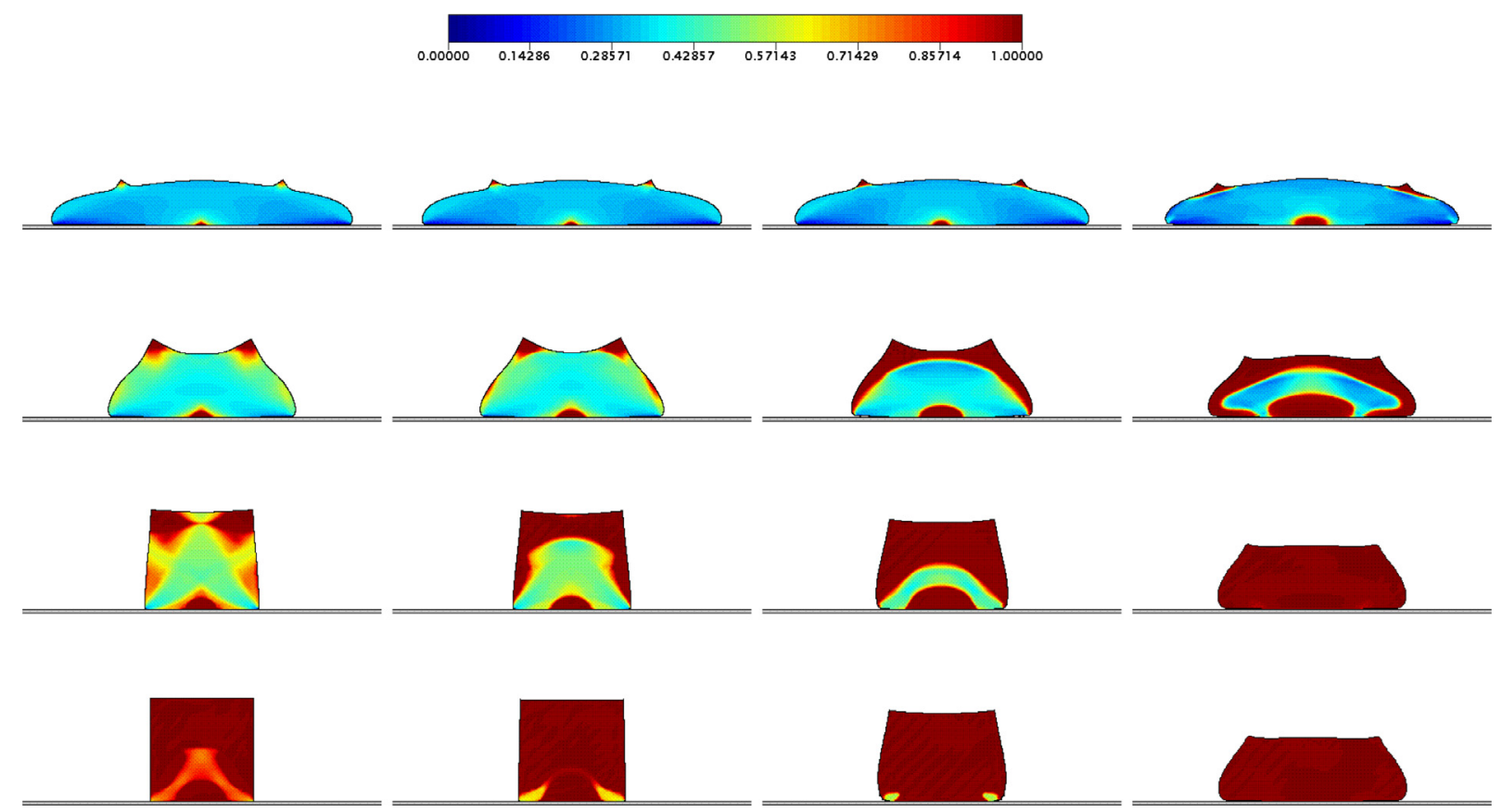

Fig. 17. Numerical prediction of the structure parameter $\lambda$ at the dimensionless time $t=100$. The equilibrium time adopted is $t_{e q}=250$. From (column) left to right: $W i=0.01,0.1,1,5$, and from (row) the top to bottom: $\tau_{y}=0.1,0.3,0.5,0.7$.

time of their shape, becoming shorter and thicker. In addition, the elastic effects contribute for a lower break down process, differently to what happened in the plane channel case. In that case, the flow is essentially viscometric while here there are viscous normal stresses due to a partially extensional character of the flow. In the blocks motions the elastic effects attenuates the viscous ones.

During this evolution in time, the blocks in higher rows, corresponding to the lower values of the dimensionless thixotropic equilibrium time, experience more pronounced decrease in the structure level when compared to the blocks 

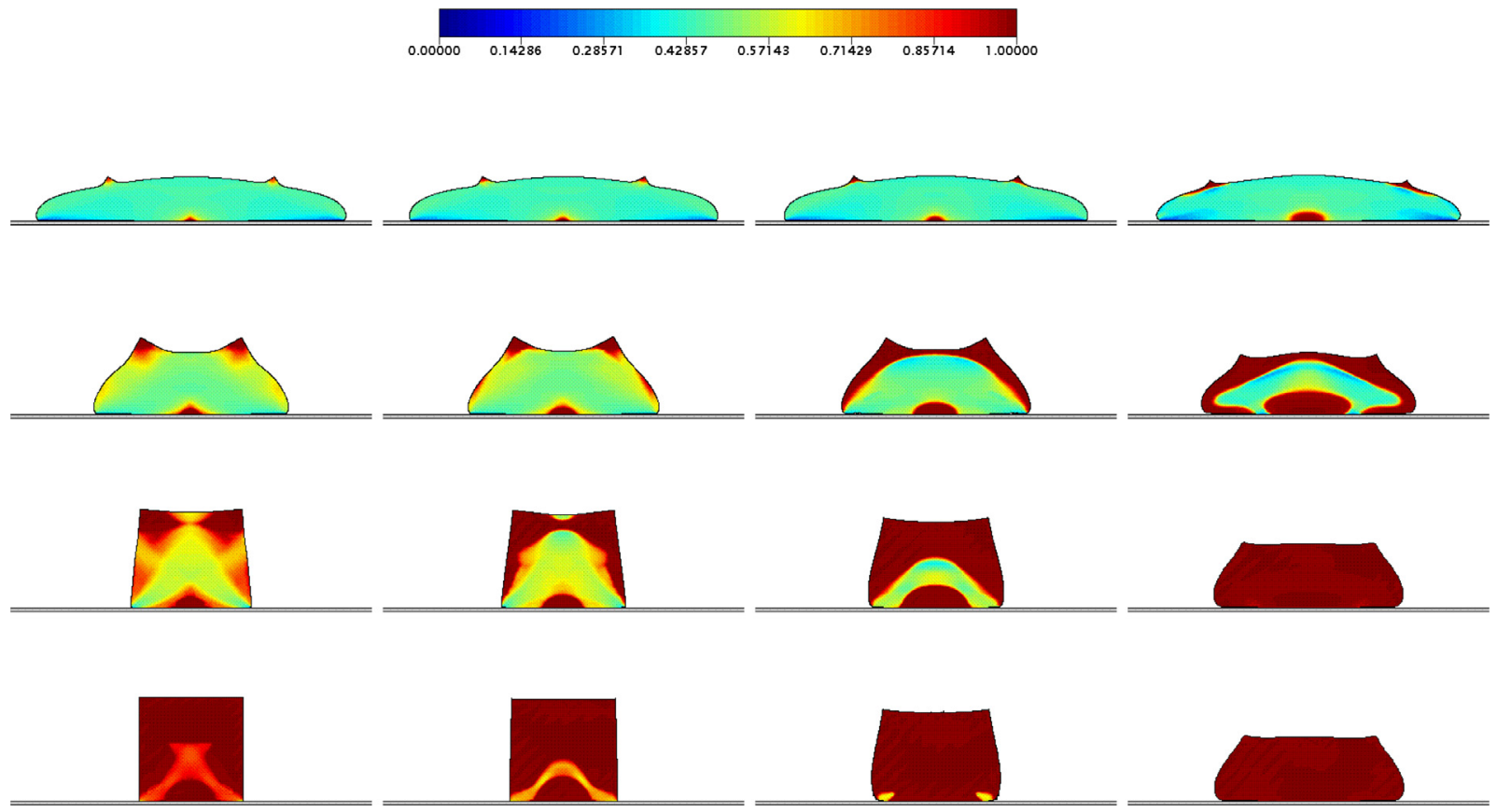

Fig. 18. Numerical prediction of the structure parameter $\lambda$ at the dimensionless time $t=150$. The equilibrium time adopted is $t_{e q}=250$. From (column) left to right: $W i=0.01,0.1,1,5$, and from (row) the top to bottom: $\tau_{y}=0.1,0.3,0.5,0.7$.

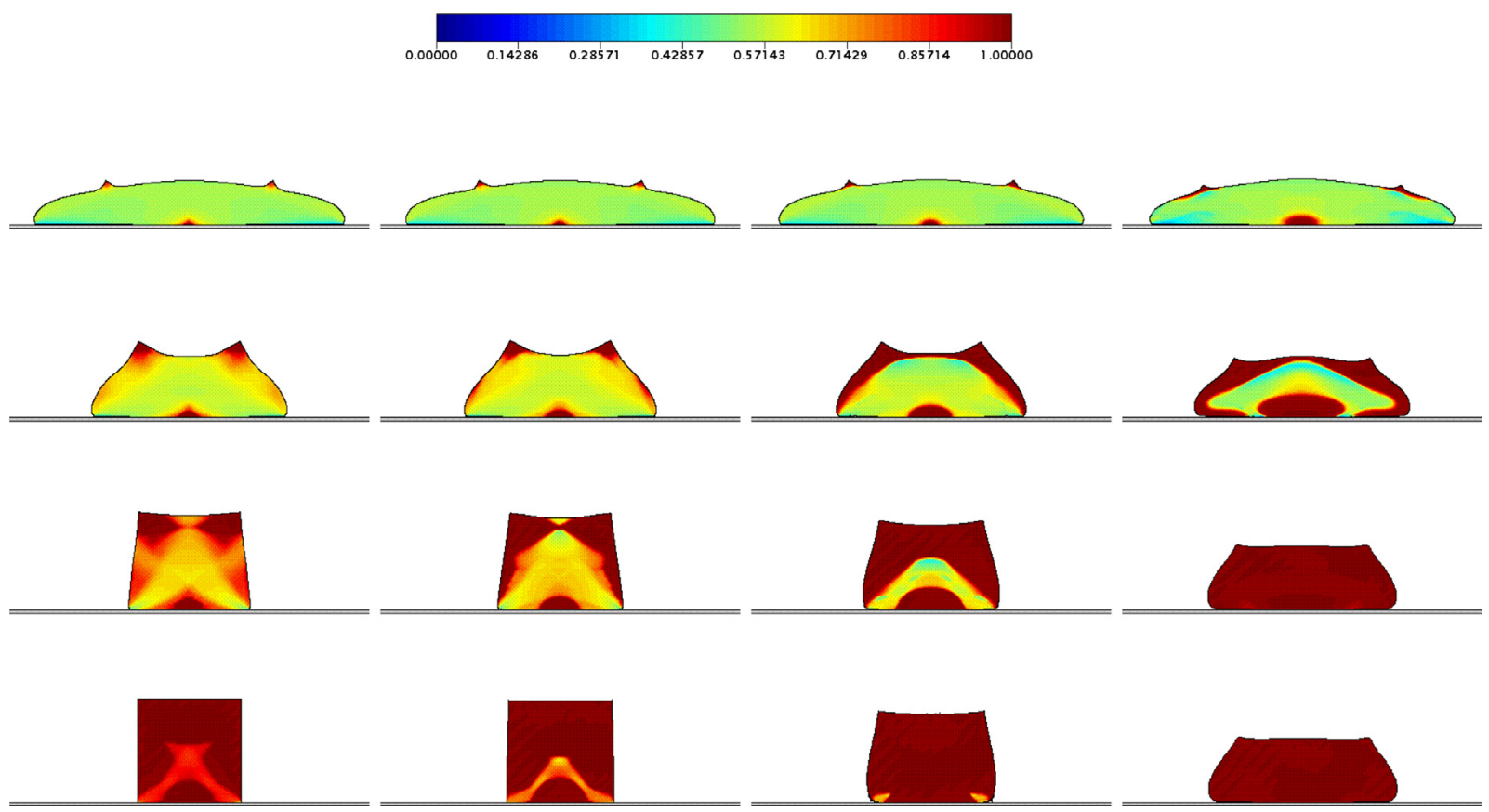

Fig. 19. Numerical prediction of the structure parameter $\lambda$ at the dimensionless time $t=200$. The equilibrium time adopted is $t_{e q}=250$. From (column) left to right: $W i=0.01,0.1,1,5$, and from (row) the top to bottom: $\tau_{y}=0.1,0.3,0.5,0.7$.

in lower rows. This is an expected result, since lower $t_{e q}$ values result in more rapid change of structure. With the exception of the highest Wi case, from time $t=10$ to $t=20$, Figs. 23 and 24 the $t_{e q}=100$ blocks start a change in shape due to this destruction, which characterizes a viscous effect.

Until time $t=20$, it is worth noticing that the blocks occupying the rows corresponding to higher values of the thixotropic equilibrium time are not necessarily repeating the evolution of the lower $t_{e q}$ rows with a shifted time. For example, if we compare the evolution of the $t_{e q}=100, W i=5$ block with the $t_{e q}=1000, W i=5$ one, we see that the firsts 


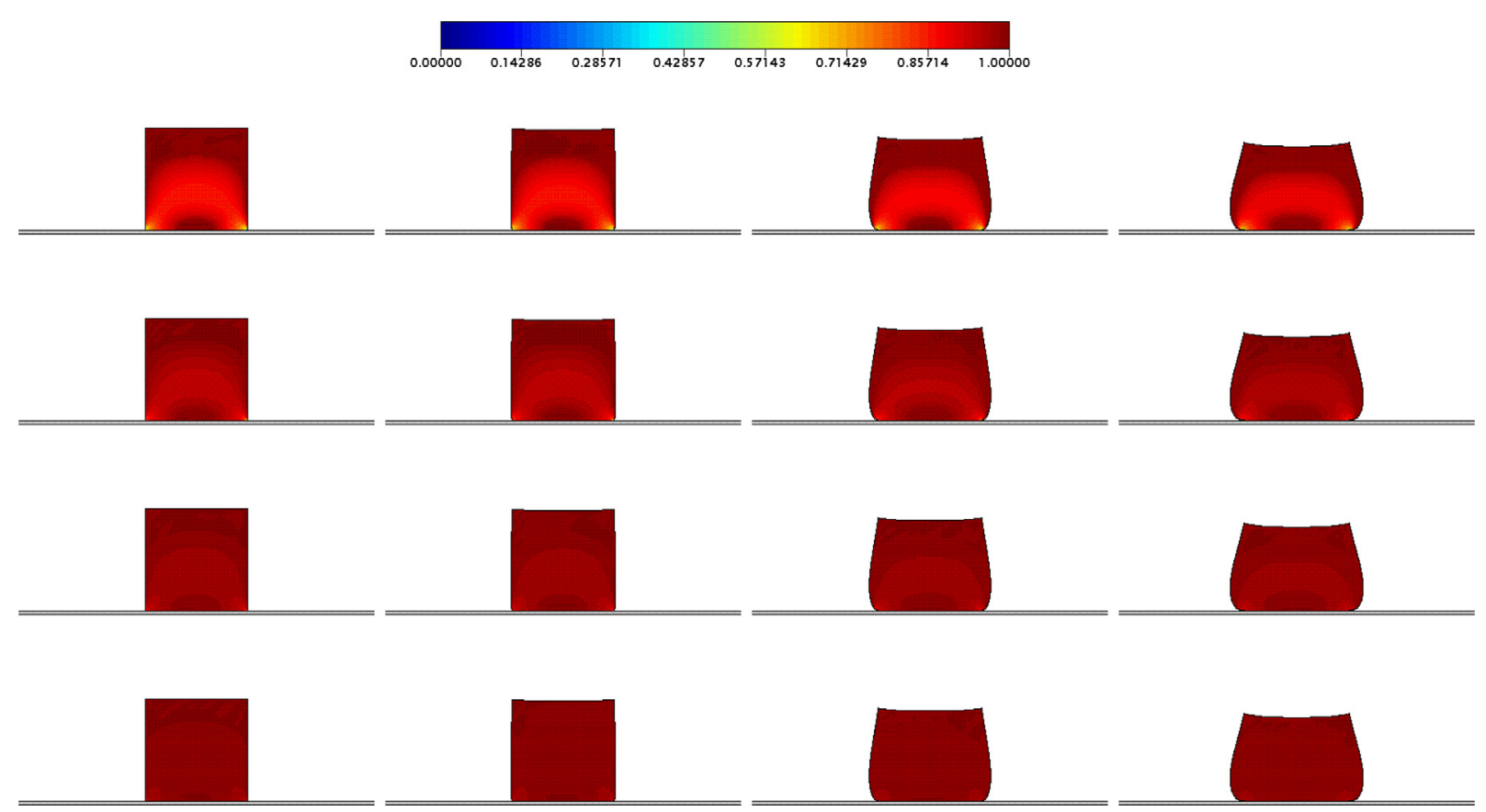

Fig. 20. Numerical prediction of the structure parameter $\lambda$ at the dimensionless time $t=1$. The yield stress adopted is $\tau_{y}=0.3$. From (column) left to right: $W i=0.01,0.1,1,5$, and from (row) the top to bottom: $t_{e q}=100,250,500,1000$.

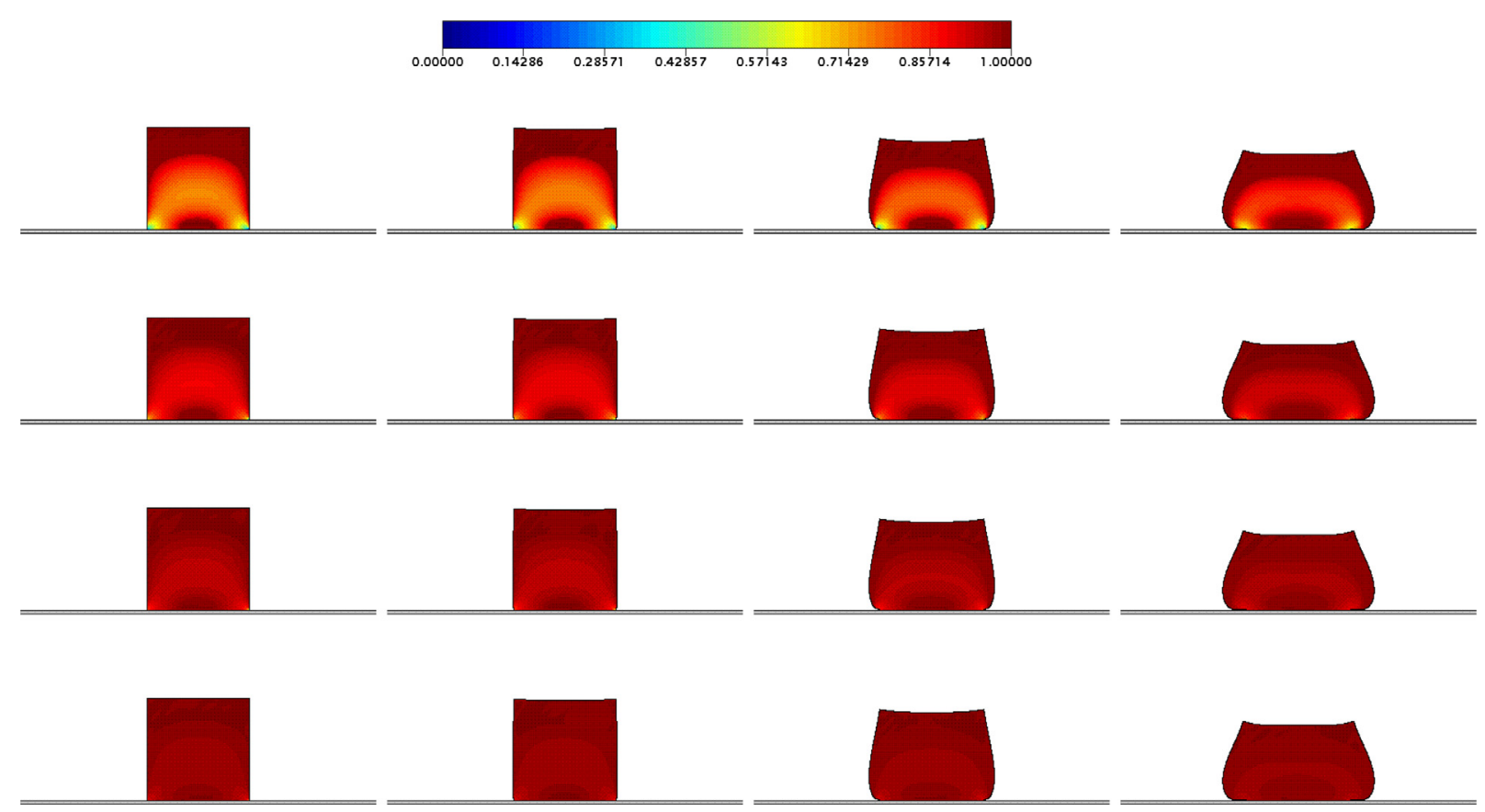

Fig. 21. Numerical prediction of the structure parameter $\lambda$ at the dimensionless time $t=2$. The yield stress adopted is $\tau_{y}=0.3$. From (column) left to right: $W i=0.01,0.1,1,5$, and from (row) the top to bottom: $t_{e q}=100,250,500,1000$.

signs of destruction in the former case occur when the block is closer to its initial shape when compared with the latter. In the long term, however, we see a more similar behavior between what is happening in the small $t_{e q}$ cases and what will happen with higher ones. Especially after time $t=50$ (Fig. 25), the blocks in the first row $\left(t_{\text {eq }}=100\right)$ experience some aging with respect to time $t=20 \mathrm{~s}$. This tendency continues to occur in the subsequent times, $t=100, t=150$, and $t=200$; corresponding to Figs. 26, 27, and 28 respectively; for this and other $t_{e q}$ values. 


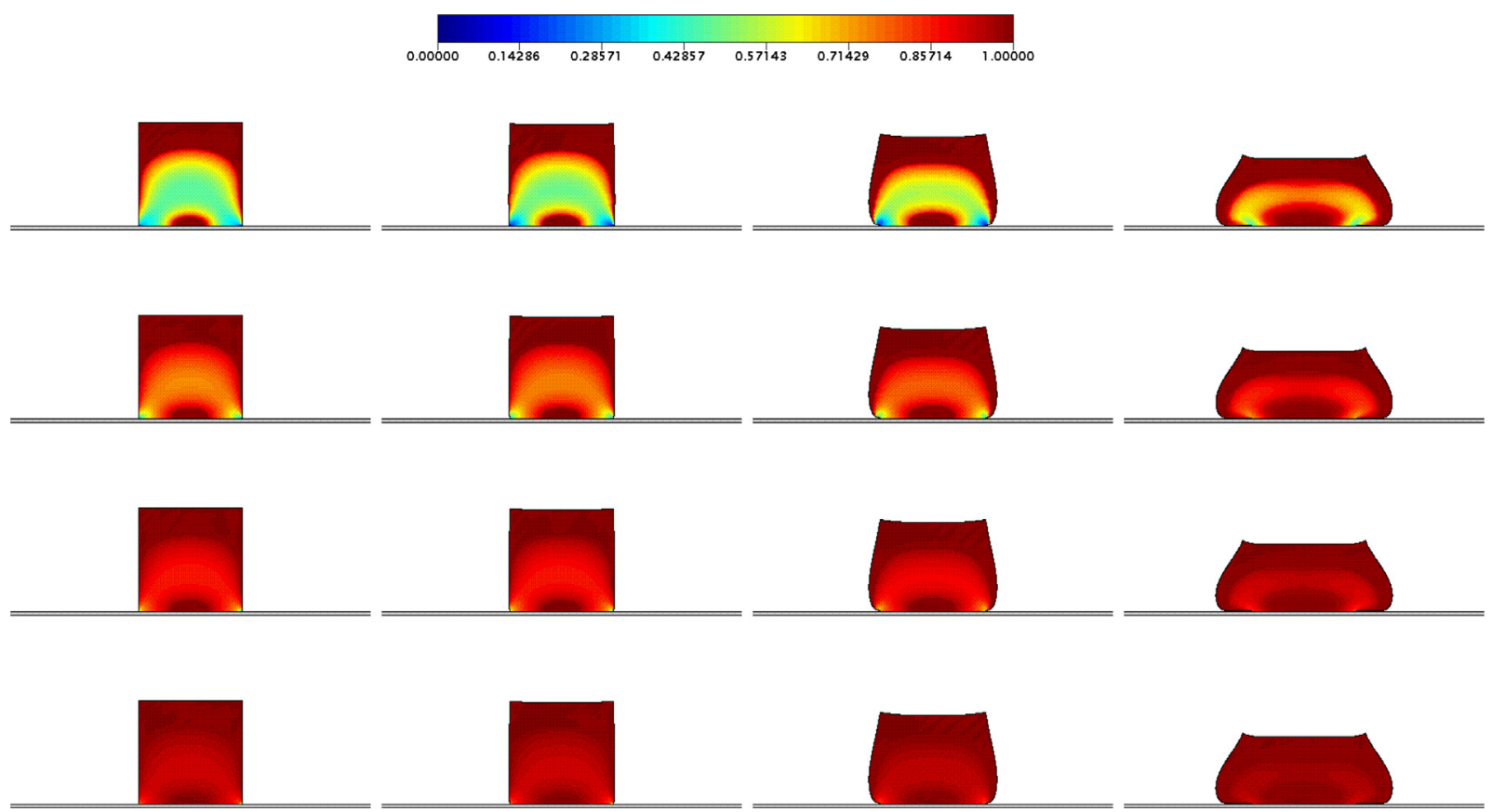

Fig. 22. Numerical prediction of the structure parameter $\lambda$ at the dimensionless time $t=5$. The yield stress adopted is $\tau_{y}=0.3$. From (column) left to right: $W i=0.01,0.1,1,5$, and from (row) the top to bottom: $t_{e q}=100,250,500,1000$.

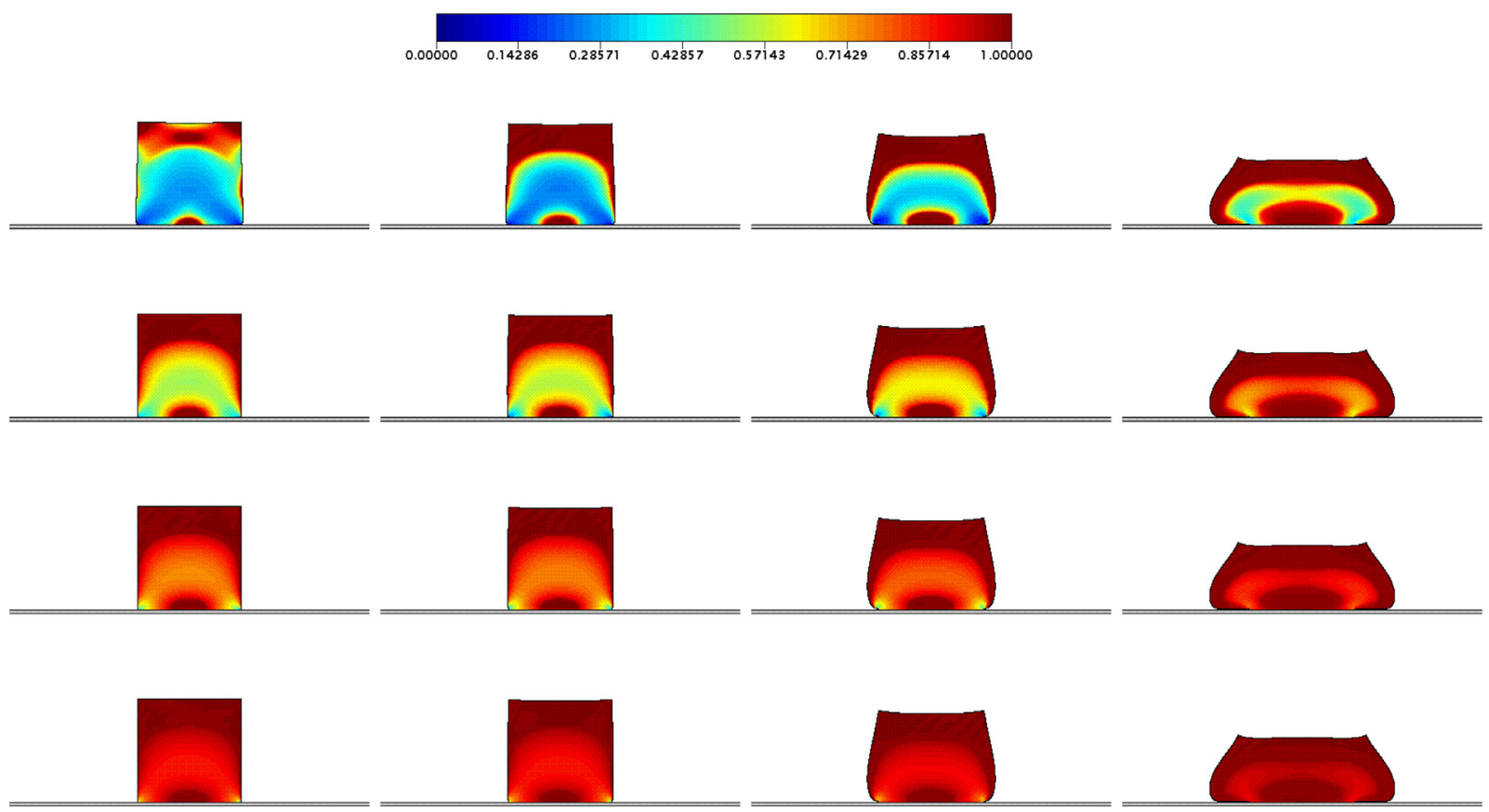

Fig. 23. Numerical prediction of the structure parameter $\lambda$ at the dimensionless time $t=10$. The yield stress adopted is $\tau_{y}=0.3$. From (column) left to right: $W i=0.01,0.1,1,5$, and from (row) the top to bottom: $t_{e q}=100,250,500,1000$.

6.2.3. Combined effects of the thixotropic equilibrium time and the yield stress tensor

Finally, we have also investigated the combined effects of the thixotropic equilibrium time and the yield stress in the dynamic of the structure parameters performing simulations at a fixed value of the Weissenberg number, $W i=0.1$. Results for dimensionless times $t=1,2,5,10,20$ are presented in Figs. 29-33, respectively.

As expected, the lower is the yield stress and the lower is the thixotropic equilibrium time, more pronounced is the destruction for a fixed time in the evolution trajectory. 


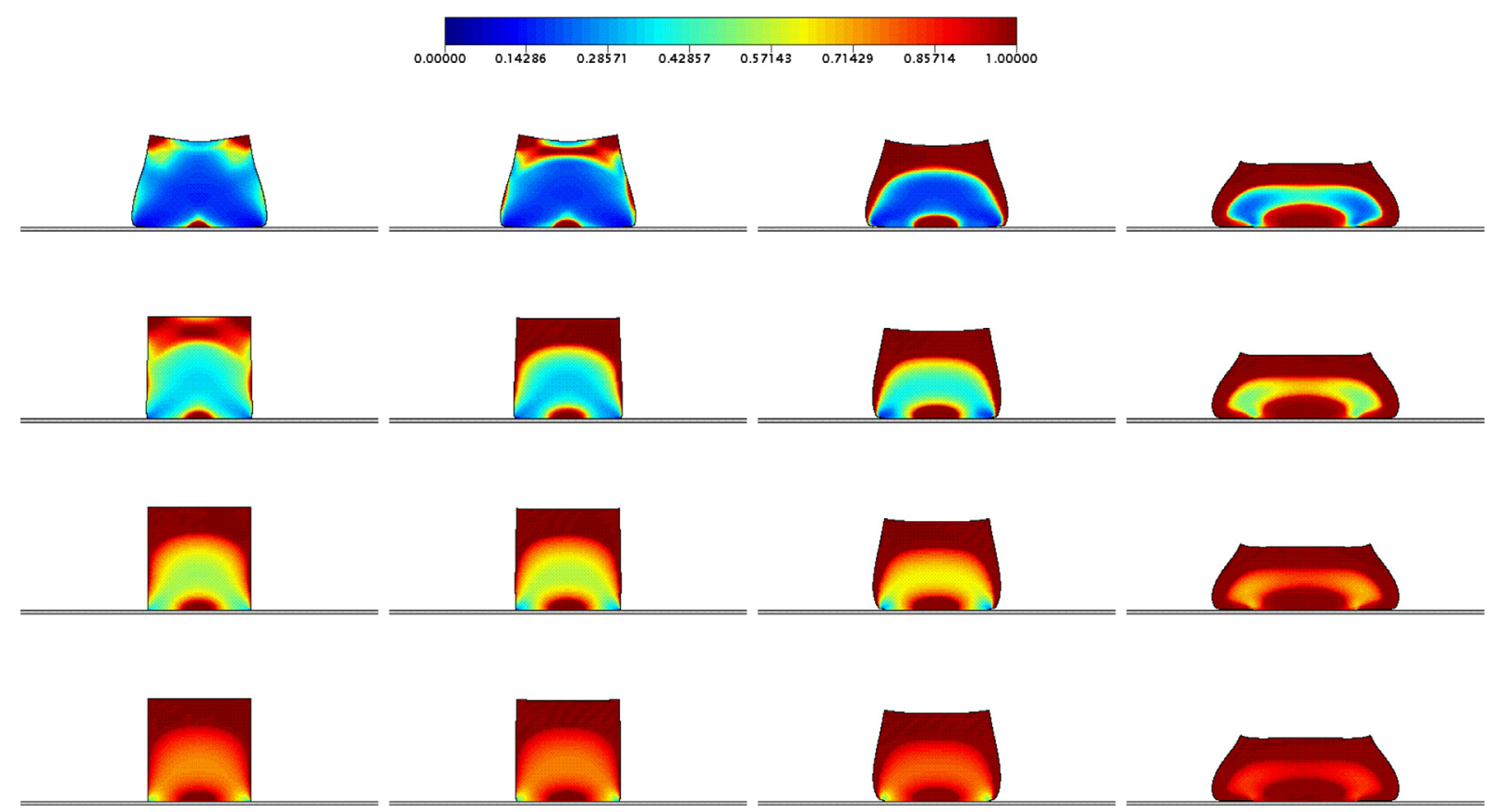

Fig. 24. Numerical prediction of the structure parameter $\lambda$ at the dimensionless time $t=20$. The yield stress adopted is $\tau_{y}=0.3$. From (column) left to right: $W i=0.01,0.1,1,5$, and from (row) the top to bottom: $t_{\text {eq }}=100,250,500,1000$.

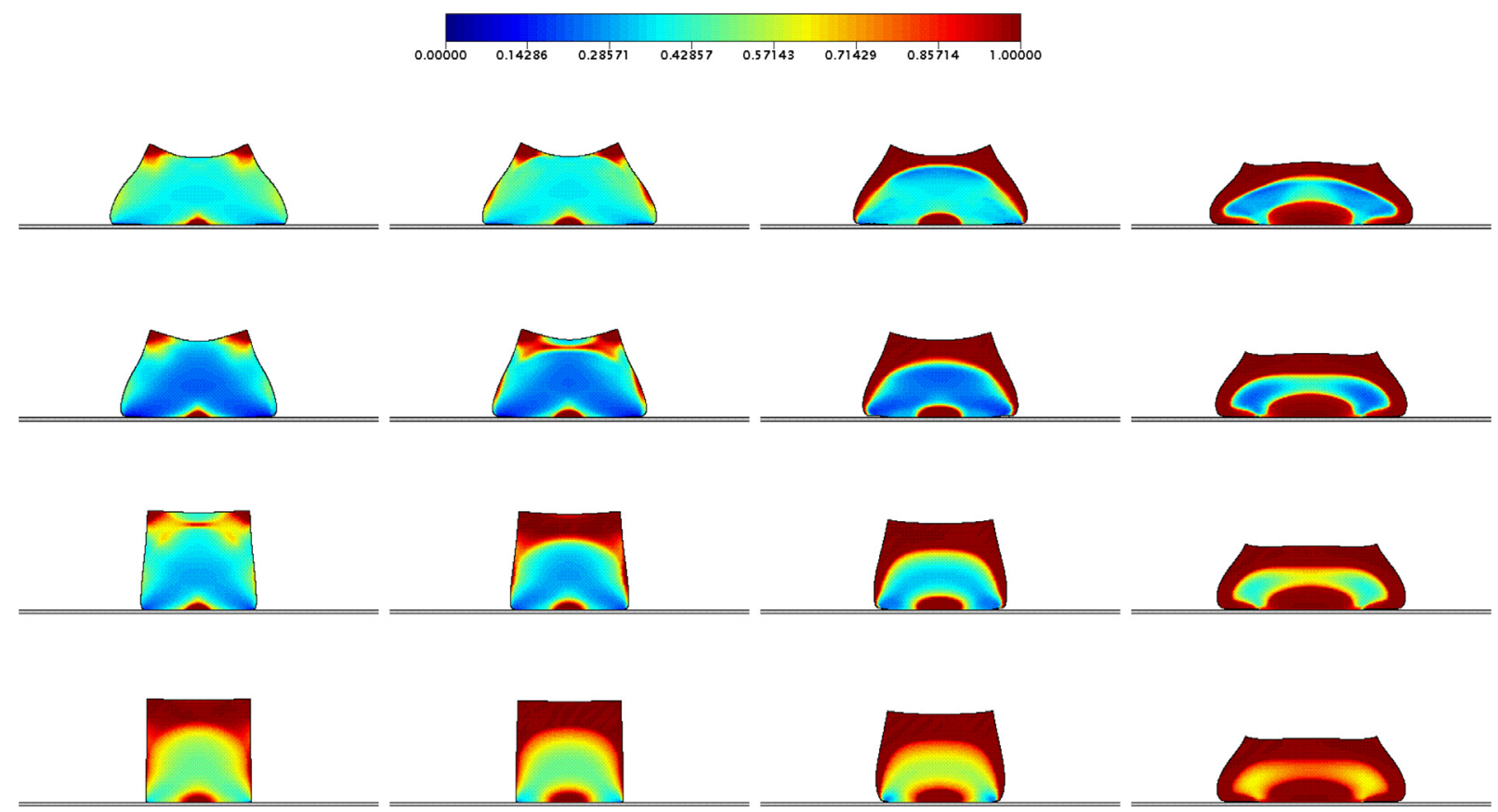

Fig. 25. Numerical prediction of the structure parameter $\lambda$ at the dimensionless time $t=50$. The yield stress adopted is $\tau_{y}=0.3$. From (column) left to right: $W i=0.01,0.1,1,5$, and from (row) the top to bottom: $t_{e q}=100,250,500,1000$.

Due to the rigidity inherent to the Weissenberg number value considered, there are practically no signs of elastic changes in shape during the whole evolution. This same fact is responsible for a coincidence between the values of the structure parameter between two blocks with the same $\tau_{y}$ at one time with a $t_{e q}$ value and a subsequent time with a higher thixotropic equilibrium time value. In other words, we see a shifted in time behavior between these blocks.

Viscous changes in shape due to the flow of the material are detected in the $\tau_{y}=0.1, t_{e q}=100$ at $t=5$, see Fig. 31 . Fig. 32 shows that, at time $t=10$, this block stars a liquid-like evolution in time, which is followed by the rows corre- 


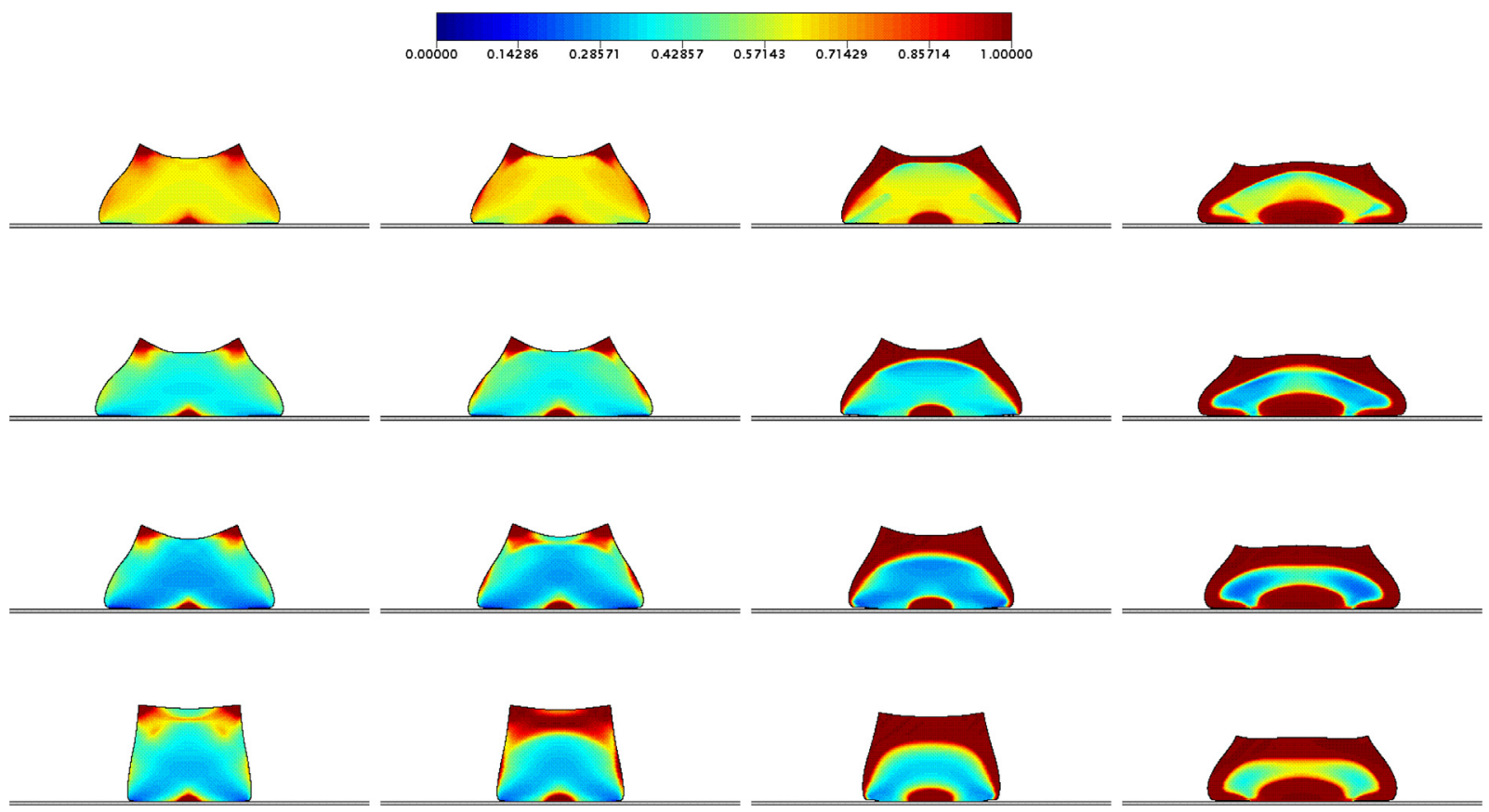

Fig. 26. Numerical prediction of the structure parameter $\lambda$ at the dimensionless time $t=100$. The yield stress adopted is $\tau_{y}=0.3$. From (column) left to right: $W i=0.01,0.1,1,5$, and from (row) the top to bottom: $t_{e q}=100,250,500,1000$.

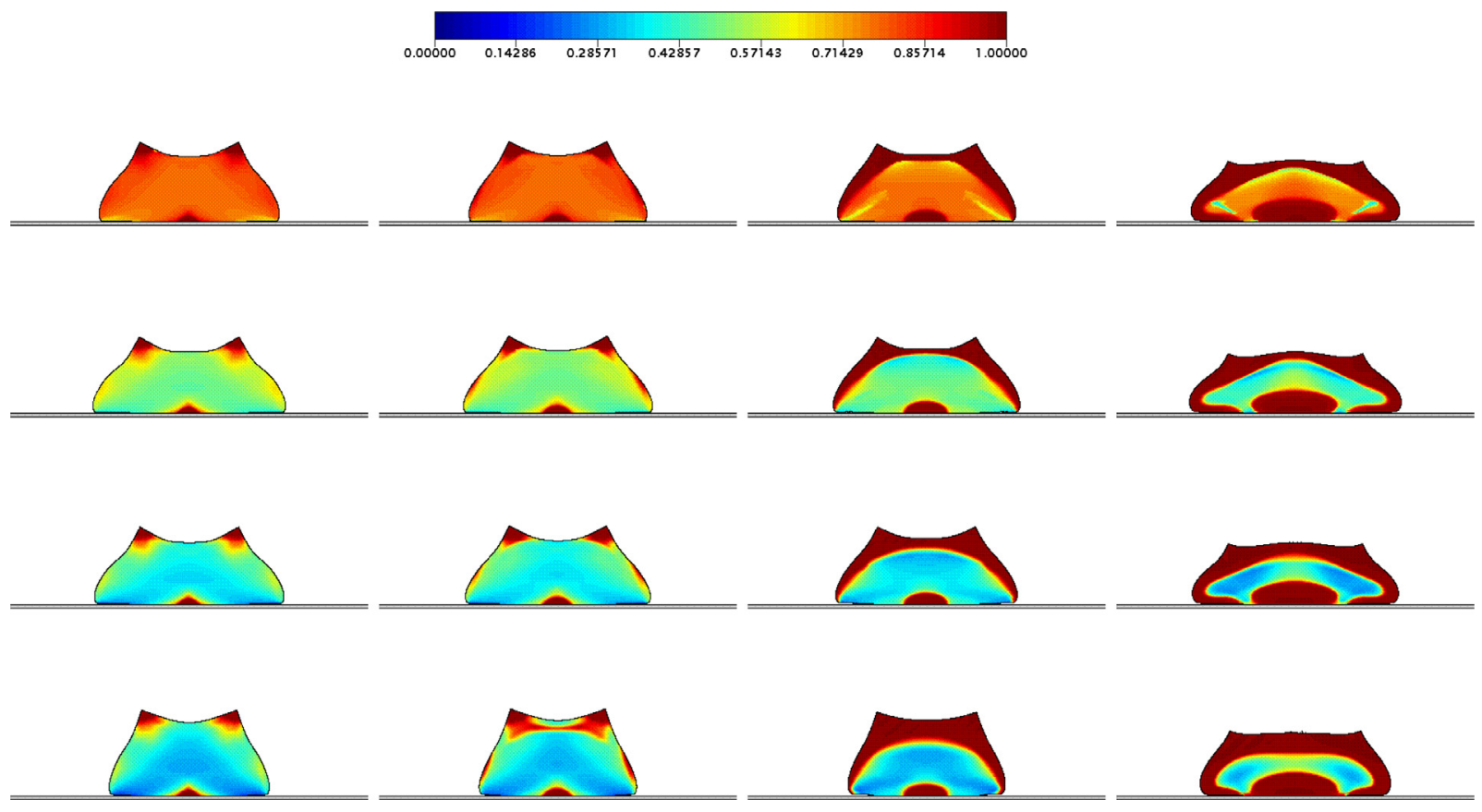

Fig. 27. Numerical prediction of the structure parameter $\lambda$ at the dimensionless time $t=150$. The yield stress adopted is $\tau_{y}=0.3$. From (column) left to right: $W i=0.01,0.1,1,5$, and from (row) the top to bottom: $t_{e q}=100,250,500,1000$.

sponding to subsequent values of $t_{e q}$ in this same yield stress value. On the other hand, only at time $t=20$ (see Fig. 33) the $\tau_{y}=0.3, t_{e q}=100$ case manifests this kind of shape change. Changing in the structure parameter for lower values of $\tau_{y}$ occur even for the highest equilibrium thixotropic time value, $t_{e q}=1000$. For the highest value of yield stress, $\tau_{y}=0.7$, the blocks remain in their original shape even having signs of rejuvenation at the bottom near the free surface. 


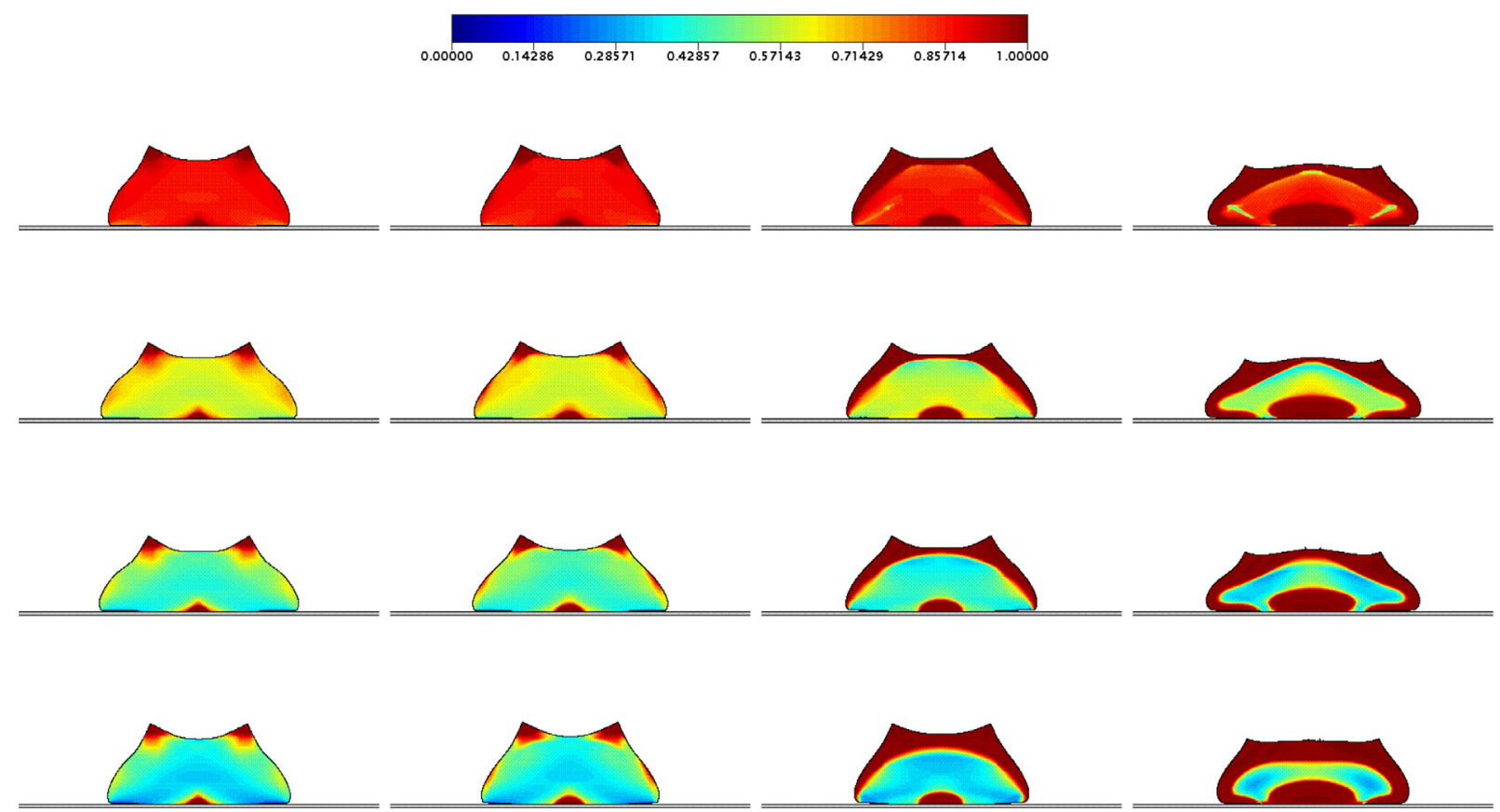

Fig. 28. Numerical prediction of the structure parameter $\lambda$ at the dimensionless time $t=200$. The yield stress adopted is $\tau_{y}=0.3$. From (column) left to right: $W i=0.01,0.1,1,5$, and from (row) the top to bottom: $t_{e q}=100,250,500,1000$.

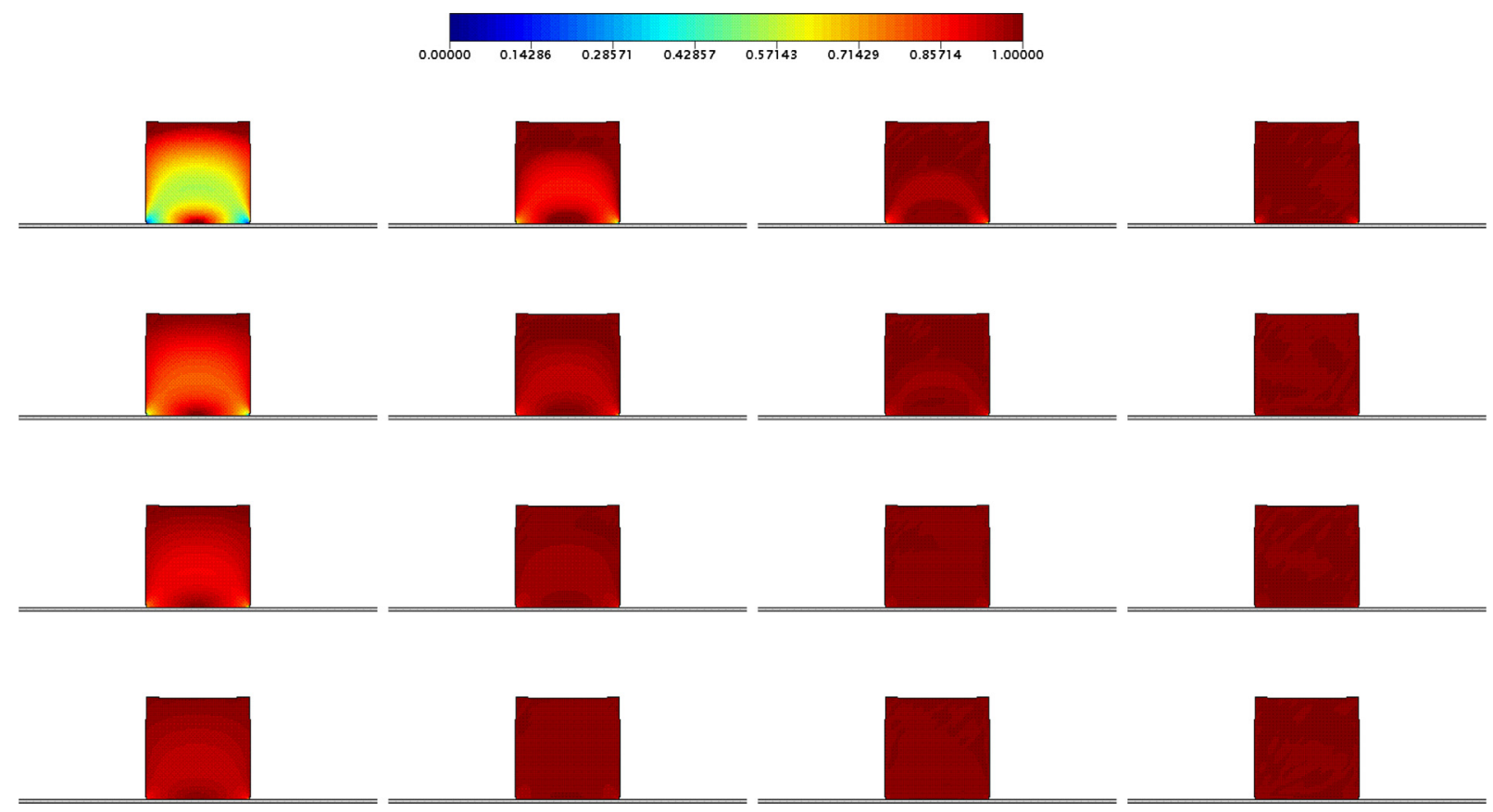

Fig. 29. Numerical prediction of the structure parameter $\lambda$ at the dimensionless time $t=1$. The Weissenberg number adopted is $W i=0.1$. From (column) left to right: $\tau_{y}=0.1,0.3,0.5,0.7$, and from (row) the top to bottom: $t_{e q}=100,250,500,1000$.

Time $t=50$ (Fig. 34) shows highly flat shapes for the $\tau_{y}=0.1$-low- $t_{\text {eq }}$ cases. This figure also presents aging for the cases $t_{e q}=100-\tau_{y}=0.1, t_{e q}=100-\tau_{y}=0.3$, and $t_{e q}=250-\tau_{y}=0.1$. Aging progresses in a down-main-diagonal direction, i.e. from low- $\tau_{y}$-low- $t_{e q}$ to high- $\tau_{y}$-high- $t_{e q}$ cases for subsequent time values of $t=100, t=150$, and $t=200$; associated to Figs. 35, 36, and 37, respectively. 


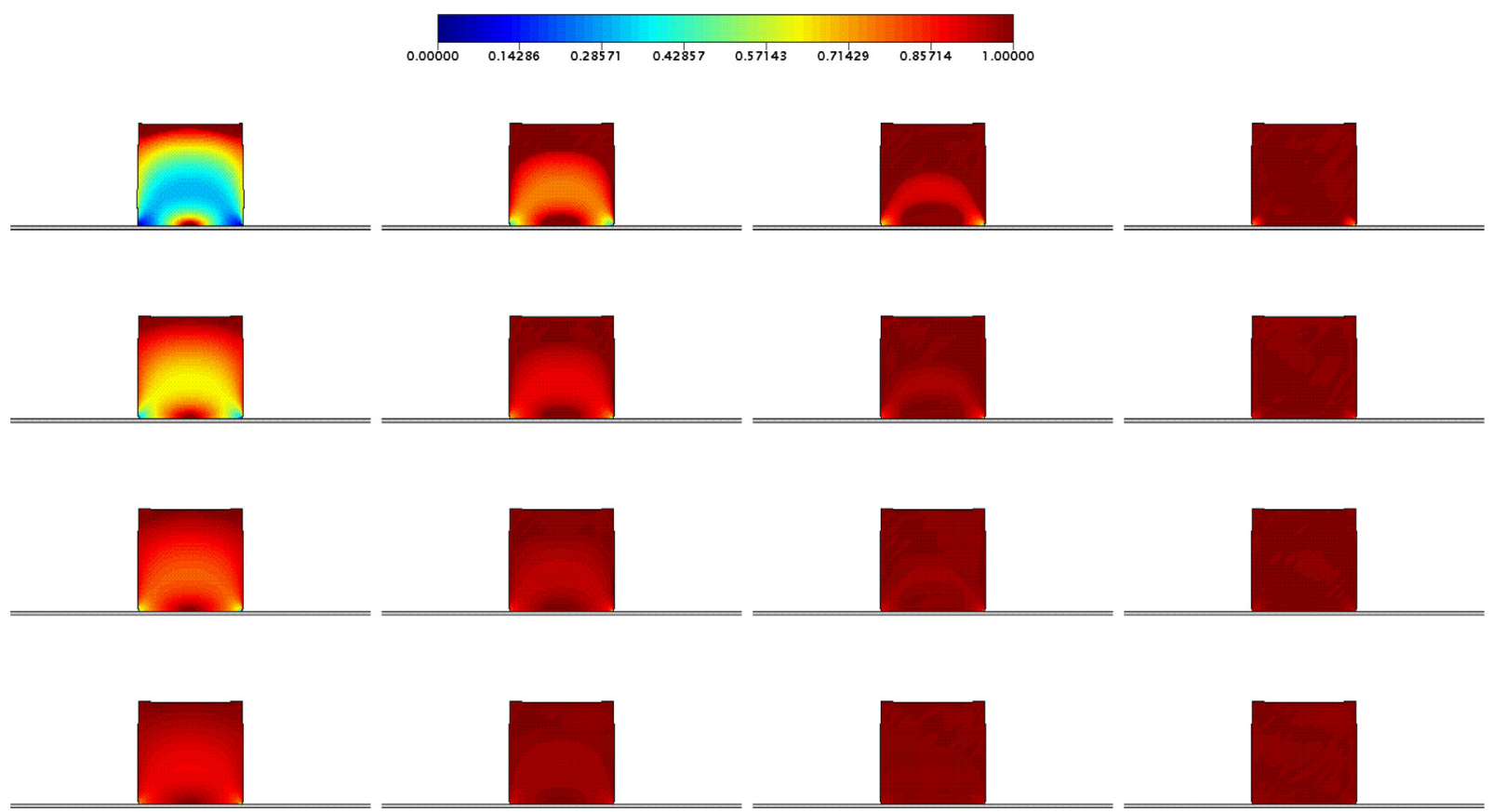

Fig. 30. Numerical prediction of the structure parameter $\lambda$ at the dimensionless time $t=2$. The Weissenberg number adopted is $W i=0.1$. From (column) left to right: $\tau_{y}=0.1,0.3,0.5,0.7$, and from (row) the top to bottom: $t_{\text {eq }}=100,250,500,1000$.

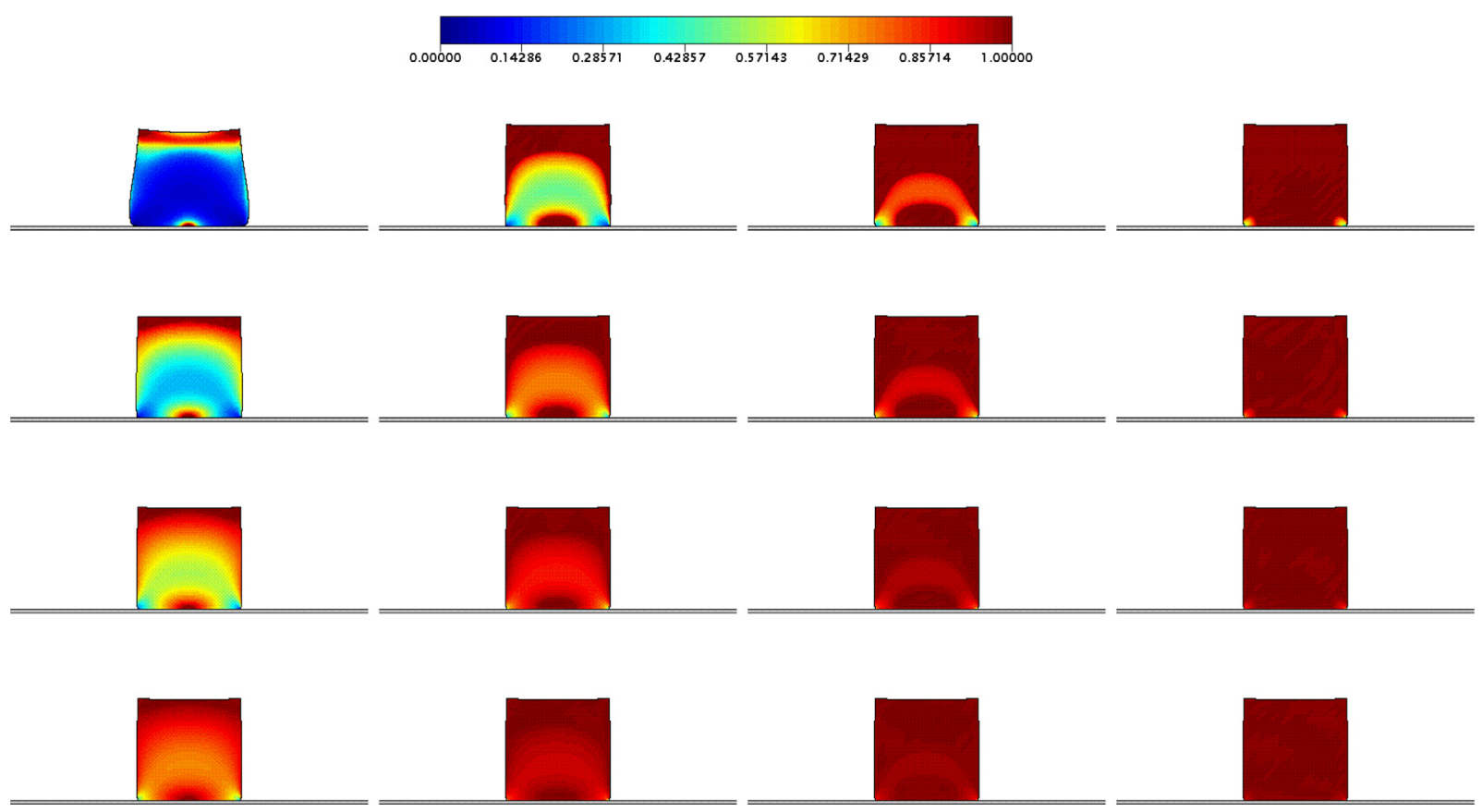

Fig. 31. Numerical prediction of the structure parameter $\lambda$ at the dimensionless time $t=5$. The Weissenberg number adopted is $W i=0.1$. From (column) left to right: $\tau_{y}=0.1,0.3,0.5,0.7$, and from (row) the top to bottom: $t_{e q}=100,250,500,1000$.

\section{Final remarks}

In the present work we developed a numerical methodology that is able to address the flow of an elasto-viscoplastic thixotropic material subjected to free surface boundary conditions. Using a finite-difference scheme, the numerical formulation was based on the projection method for solving the governing equations for mass and momentum while the constitutive equation for the non-Newtonian tensor was treated by the second order Runge-Kutta method. Additionally, the 


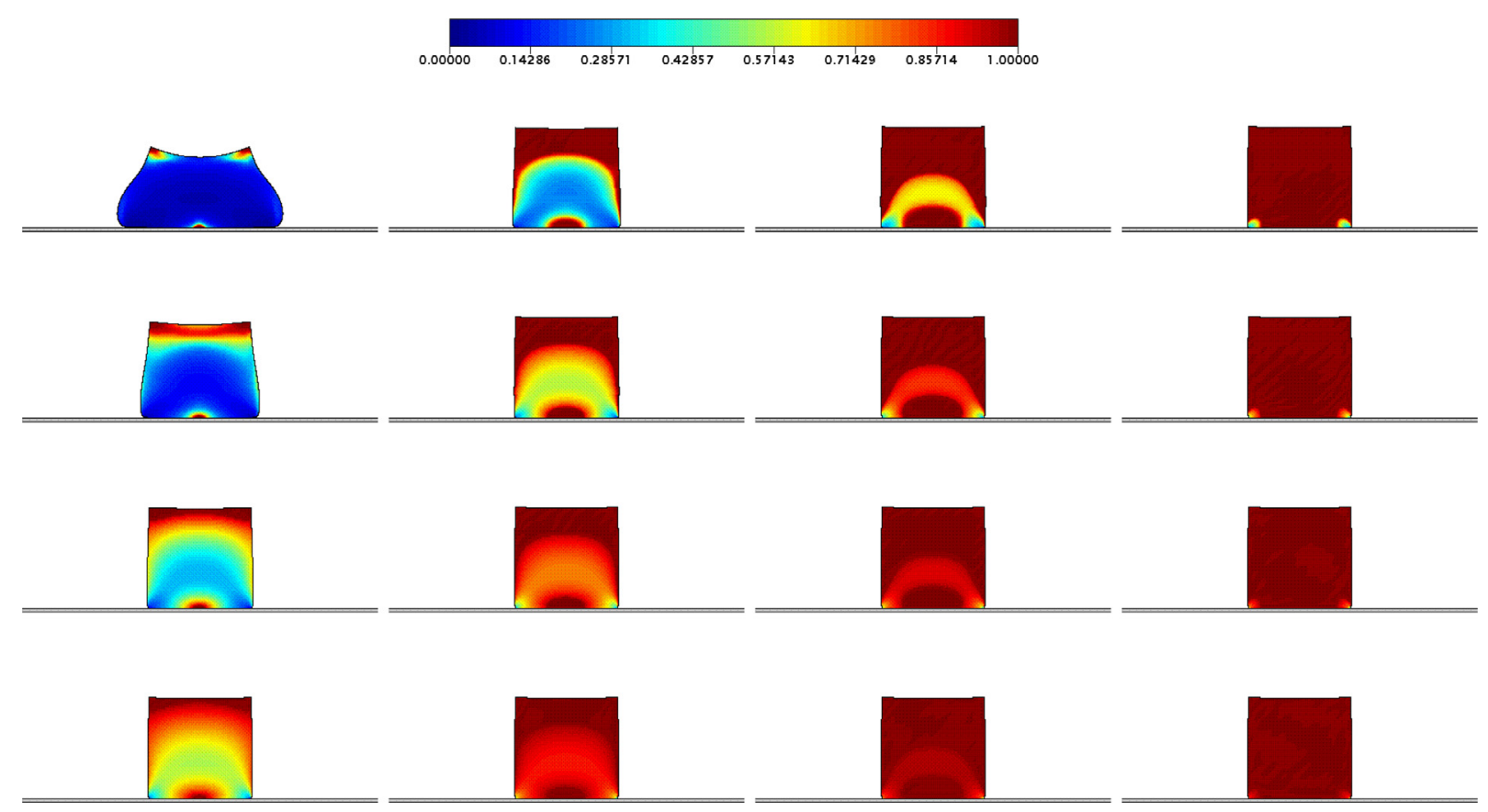

Fig. 32. Numerical prediction of the structure parameter $\lambda$ at the dimensionless time $t=10$. The Weissenberg number adopted is $W i=0.1$. From (column) left to right: $\tau_{y}=0.1,0.3,0.5,0.7$, and from (row) the top to bottom: $t_{e q}=100,250,500,1000$.

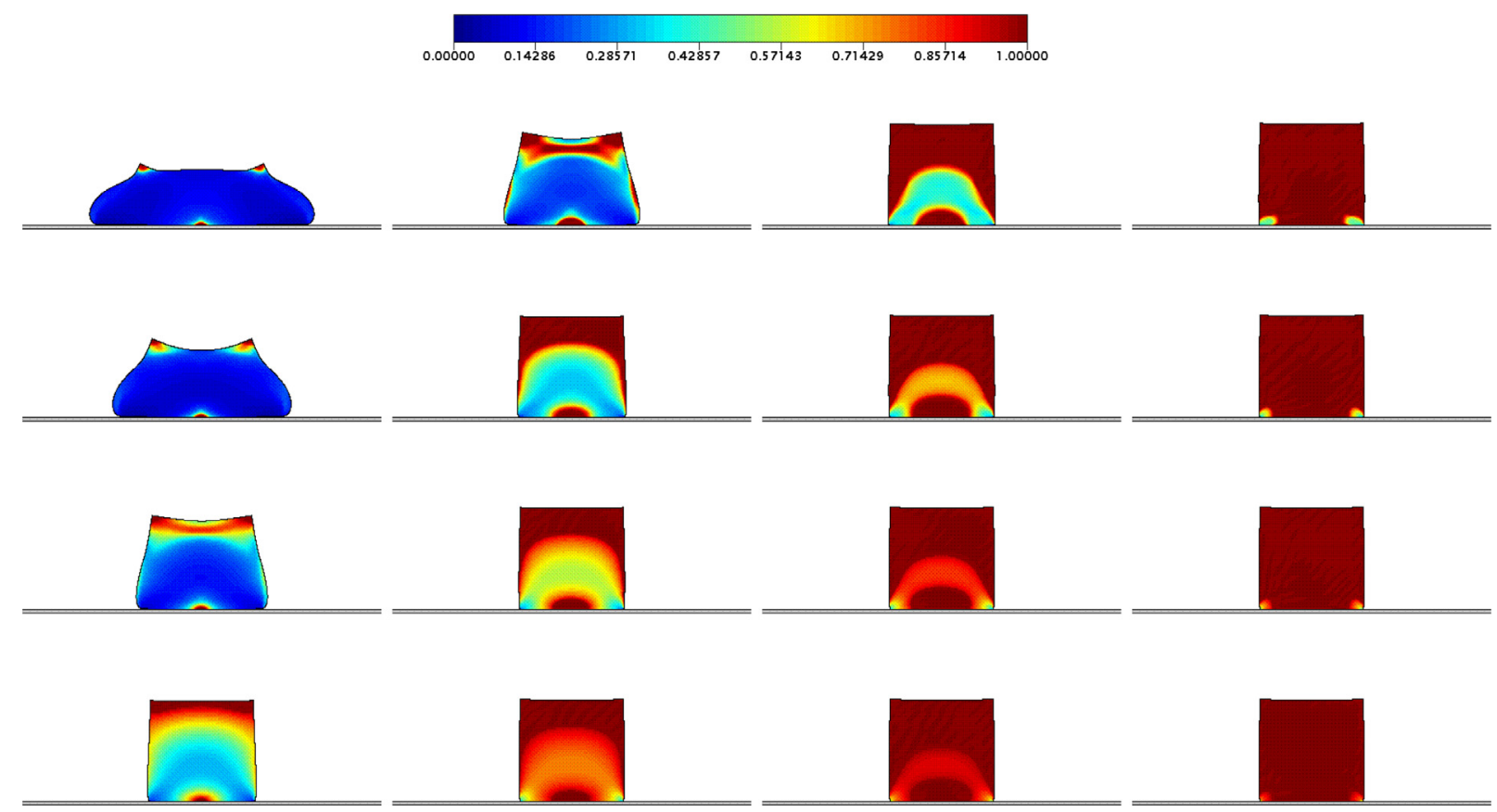

Fig. 33. Numerical prediction of the structure parameter $\lambda$ at the dimensionless time $t=20$. The Weissenberg number adopted is $W i=0.1$. From (column) left to right: $\tau_{y}=0.1,0.3,0.5,0.7$, and from (row) the top to bottom: $t_{e q}=100,250,500,1000$.

transport equation for the structure parameter, which was used to represent the thixotropic effects, was computed by a semi-implicit scheme. In order to enhance the stability of the numerical scheme in low Reynolds number moving free surface flows, the normal stress boundary condition was implicitly discretized. To deal with the two-dimensional fluid interface, the combination of the MAC method and the front-tracking framework was employed.

The present approach was also conceived to handle initial and boundary conditions that are stress-based. These kinds of conditions are particularly important in applied problems related to materials that exhibit transition from solid-like to 


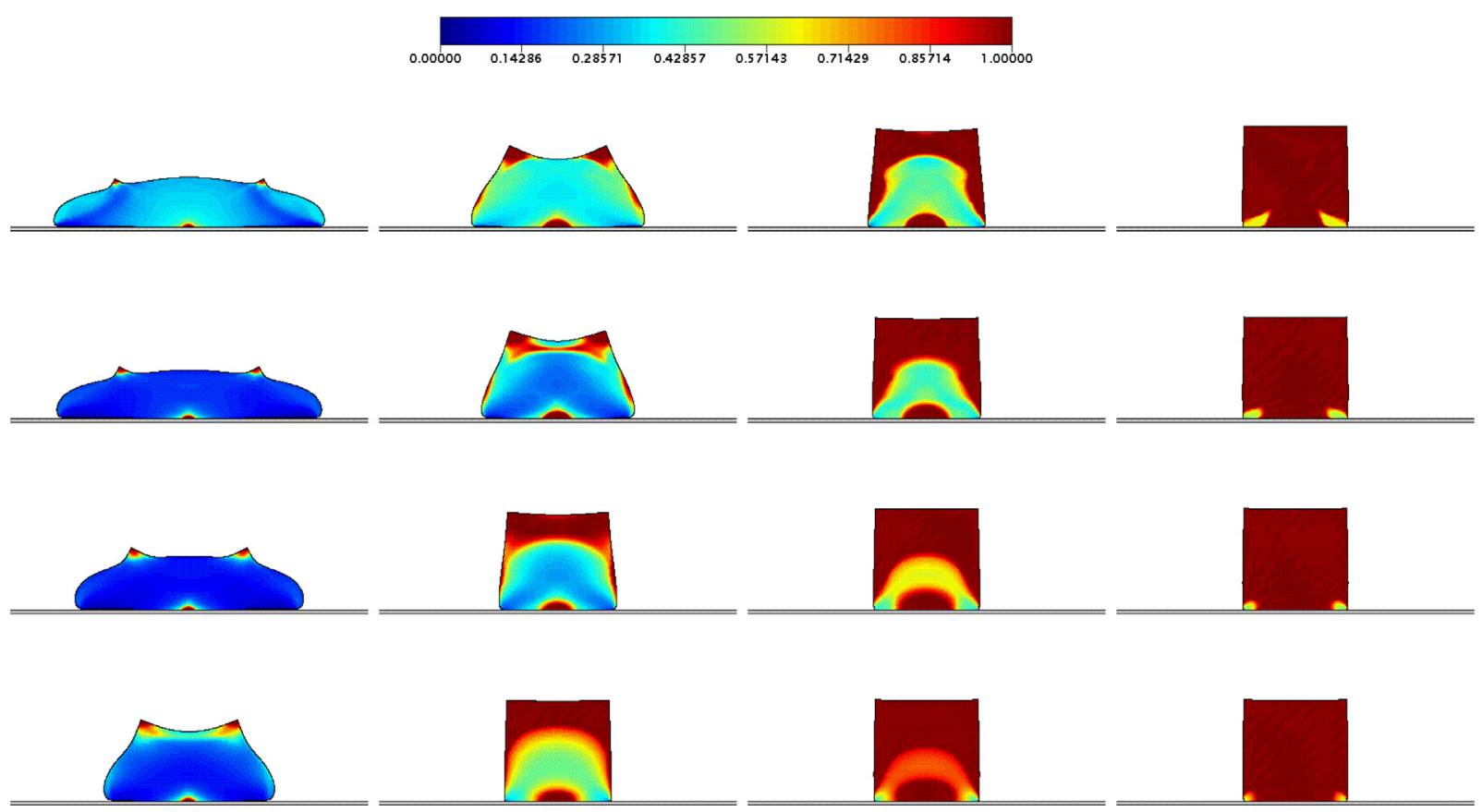

Fig. 34. Numerical prediction of the structure parameter $\lambda$ at the dimensionless time $t=50$. The Weissenberg number adopted is $W i=0.1$. From (column) left to right: $\tau_{y}=0.1,0.3,0.5,0.7$, and from (row) the top to bottom: $t_{e q}=100,250,500,1000$.

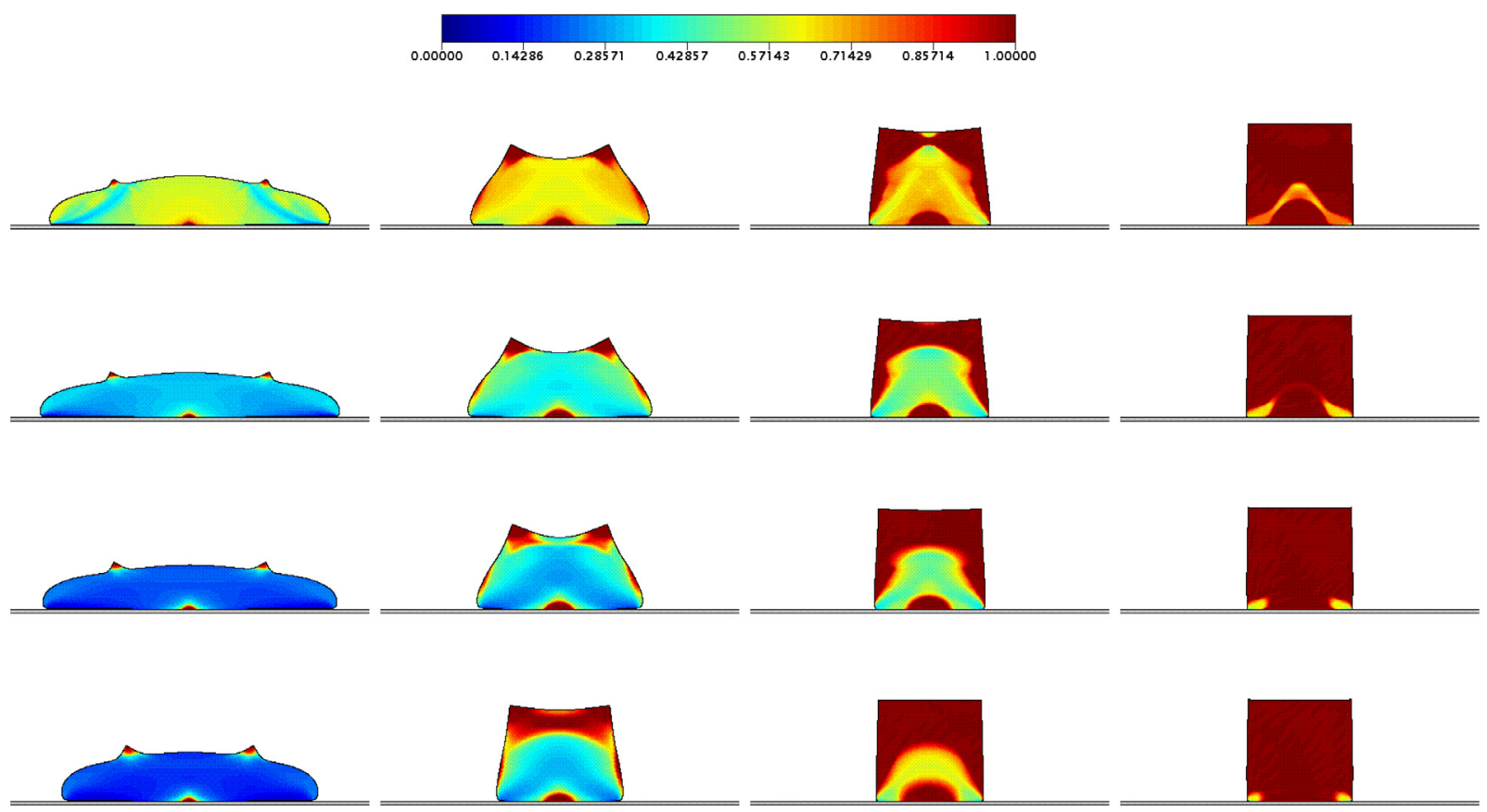

Fig. 35. Numerical prediction of the structure parameter $\lambda$ at the dimensionless time $t=100$. The Weissenberg number adopted is $W i=0.1$. From (column) left to right: $\tau_{y}=0.1,0.3,0.5,0.7$, and from (row) the top to bottom: $t_{e q}=100,250,500,1000$.

liquid-like behavior as a consequence of the imposed stress, as the ones the present method intends to model. This happens because the kind of motion the material will undergo is a direct consequence of the stress distribution along the material. In this sense, these motions do not present a a priori characteristic velocity. The constructed characteristic velocity is obtained in the present work from the characteristic stress of the problem plugged in the flow curve and expressed in an implicit manner in the complete case. The ability of the present approach to capture the transient evolution of the material motion is especially important when one is dealing with thixotropic systems where a time scale associated to the structure change 


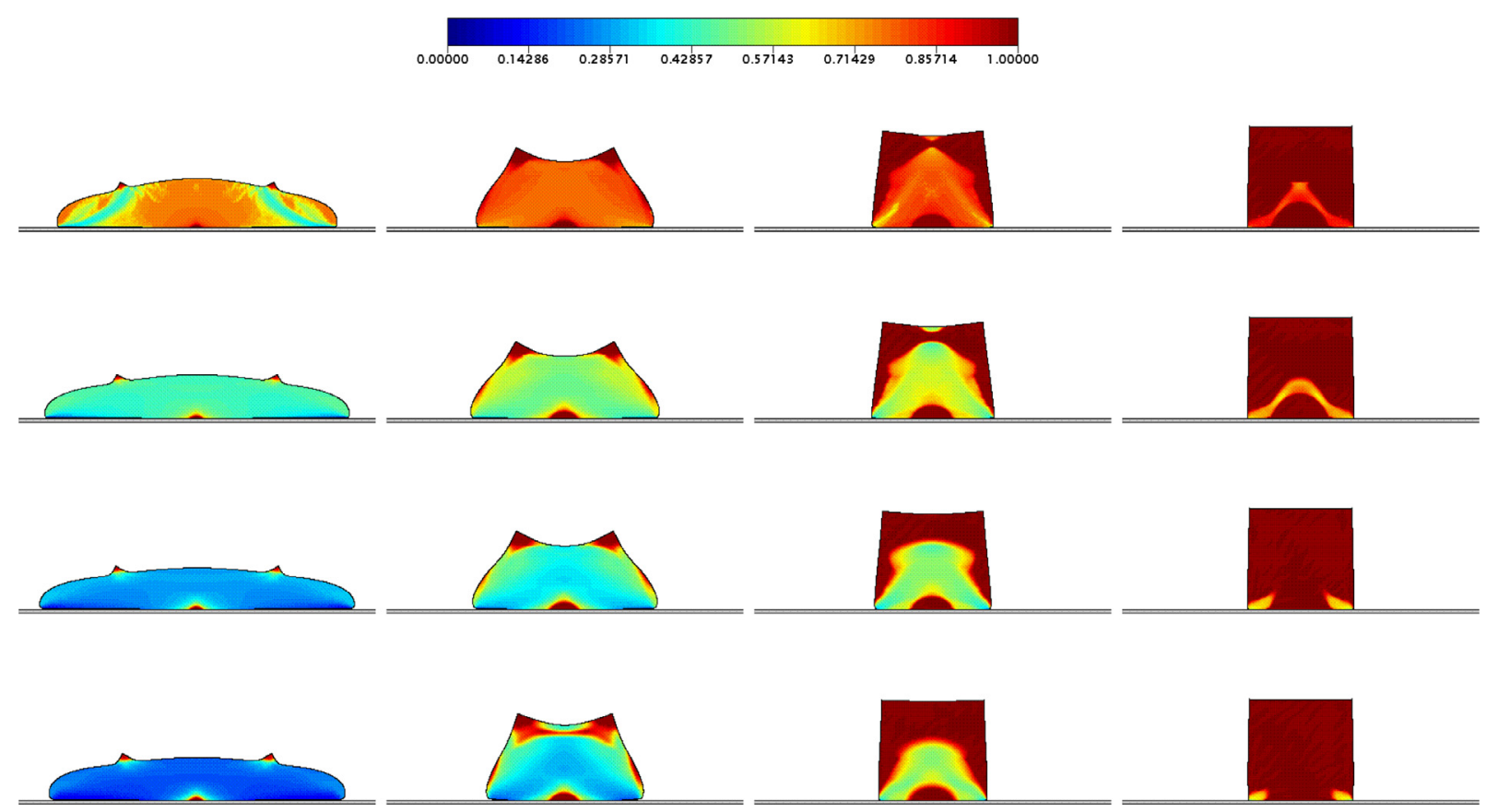

Fig. 36. Numerical prediction of the structure parameter $\lambda$ at the dimensionless time $t=150$. The Weissenberg number adopted is $W i=0.1$. From (column) left to right: $\tau_{y}=0.1,0.3,0.5,0.7$, and from (row) the top to bottom: $t_{\text {eq }}=100,250,500,1000$.

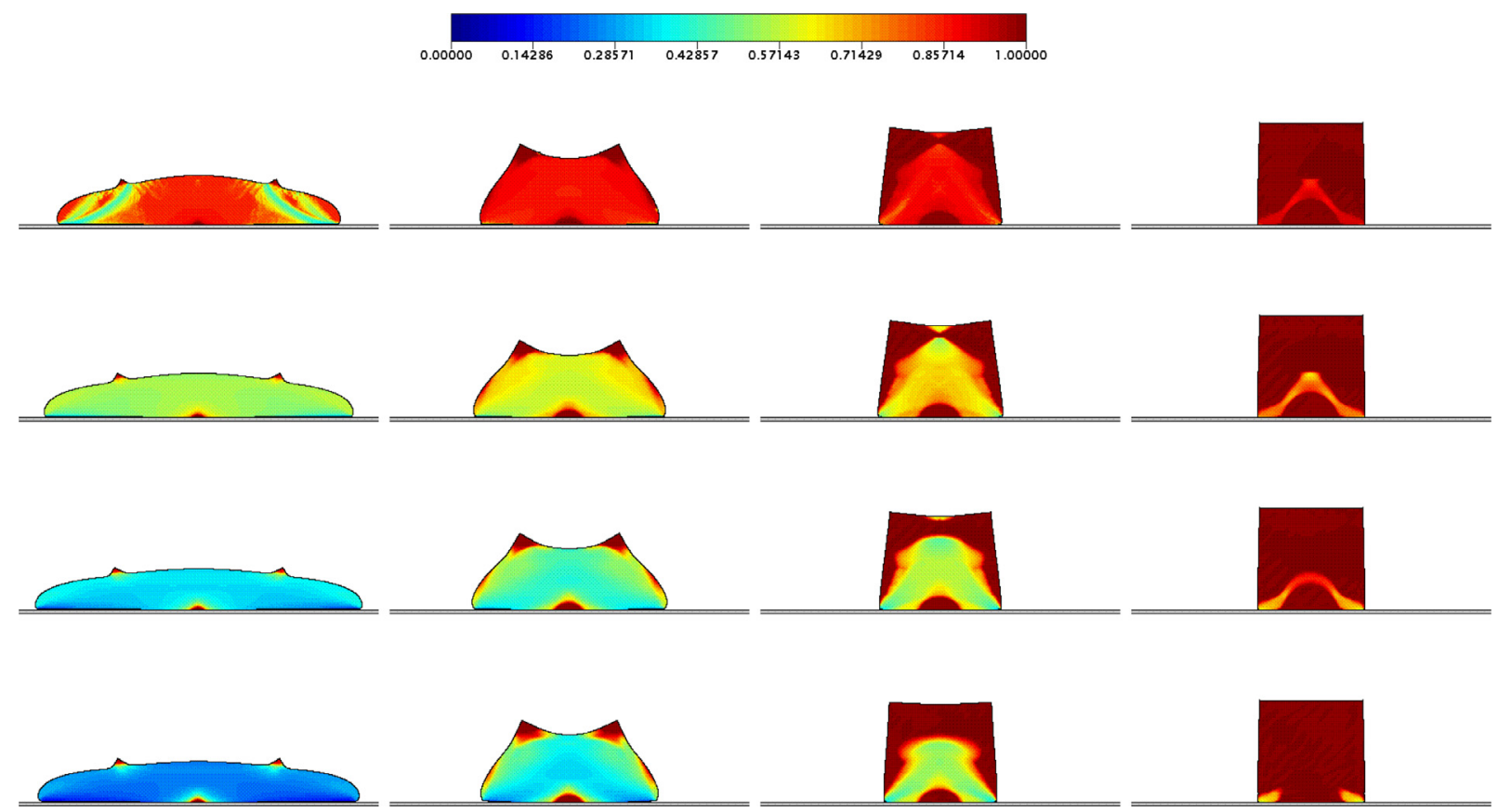

Fig. 37. Numerical prediction of the structure parameter $\lambda$ at the dimensionless time $t=200$. The Weissenberg number adopted is $W i=0.1$. From (column) left to right: $\tau_{y}=0.1,0.3,0.5,0.7$, and from (row) the top to bottom: $t_{e q}=100,250,500,1000$.

during the process is incorporated. Although the method is able to address more complex flow curves, a Bingham model with a bi-viscosity regularization method was employed to represent the apparent-yield-stress nature of the materials considered.

The flow of the elasto-viscoplastic thixotropic material was tested in a more simple geometry, the plane channel flow subjected to a constant pressure gradient. We stress that no flow rate is provided as initial or boundary conditions. A fullystructured state was imposed as initial condition and Bingham-like velocity profiles were obtained in steady state, with 
a plug region dependent on the dimensionless yield stress, as expected. The evolution in time of the structure parameter presented an expected dependence on the thixotropic equilibrium time, i.e. a higher delay between imposed stress and the rejuvenation process for higher values of $t_{\text {eq }}$. Normal stresses associated to the viscoelastic model also contribute to von Mises criterion for the break down process and, therefore, higher Wi numbers induce lower structuring levels. The local distribution of $W i(\lambda)$ is also provided in this problem. This quantity translates the real local relation between elastic and viscous effects and reduces to the nominal $W i$ in the plug region.

A more complex problem where the full approach presented here is explored is the rejuvenation of a block made by an elasto-viscoplastic thixotropic material initially with a fully-structured square shape, subjected to the action of gravity and to an atmospheric pressure environment. The gravity forces and the surrounding pressure conditions impose to the material a deviatoric stress state able to lead a break down process which is more pronounced at the bottom of the block. We depicted our general results in a Cartesian manner where two of the three most relevant dimensionless quantities (Weissenberg number, dimensionless yield stress, and dimensionless thixotropic equilibrium time) are varied. This way of displaying the results enables one to have a better understanding of two-by-two combined effects. Our results show that, for the cases analyzed, the time scale associated to the elastic response of the material is faster than the characteristic times scale associated to other processes. In this connection, the material that is fully-structured tends to deform elastically at the beginning of its evolution and, as expected, this deformation is more prominent at high Weissenberg numbers, due to a more flexible response attribute of the material. For the lowest value of Weissenberg number analyzed, $W i=0.01$ elastic effects were found to be negligible. Rejuvenation is dramatically high at lower dimensionless yield stress, such as $\tau_{y}=0.1$, a situation where the material exhibits a fluid-like behavior at most of the evolution process. These blocks tend to change substantially their initial square shape, increasing their base and decreasing their height significantly. At higher values of $\tau_{y}$, the material gradually acquires a more solid-like behavior what in turn results in less deformation rate due to the mobility of the material and more elastic deformation. The thixotropic equilibrium time was shown to have a significant impact on the time evolution of the material, showing a slower evolution at high $t_{e q}$ values.

Due to the complexity of the material and the problem, a number of scenarios were not simulated. Future works on elasto-viscoplastic thixotropic materials subjected to gravity forces in free-surface conditions can address other aspects of the problem. Suggestions for extensions of the investigation conducted here are: testing other evolution equations for the structure parameter, employing different values for the power-law term of the flow curve, varying the inclination angles with respect to gravity, and evaluating other aspect ratios of the block.

\section{Acknowledgements}

C.M. Oishi acknowledges the financial support of FAPESP (Fundação de Amparo à Pesquisa do Estado de São Paulo) Grant 2013/07375-0 and CNPq (Conselho Nacional de Desenvolvimento Cientifico e Tecnologico), Grants 473589/2013-3 and 309514/2013-4. R.L. Thompson acknowledges CNPq (Conselho Nacional de Desenvolvimento Cientifico e Tecnologico), Grants 485427/2012-5 and 309004/2014-4.

\section{References}

Alexandrou, A., Florides, G., \& Georgiou, G. C. (2013). Squeeze flow of semi-solid slurries. Journal of Non-Newtonian Fluid Mechanics, 193, 103-115.

Ancey, C. (2007). Plasticity and geophysical flows: A review. Journal of Non-Newtonian Fluid Mechanics, 142, 4-35.

Ancey, C., \& Cochard, S. (2009). The dam-break problem for Herschel-Bulkley viscoplastic fluids down steep flumes. Journal of Non-Newtonian Fluid Mechanics, $158,18-35$

Balmforth, N. J., Frigaard, I., \& Ovarlez, G. (2014). Yielding to stress: Recent developments in viscoplastic fluid mechanics. Annual Review of Fluid Mechanics, $46,45-64$.

Balmforth, N. J., Craster, R. V., Perona, P., Rust, A. C., \& Sassi, R. (2007). Viscoplastic dam breaks and the bostwick consistometer. Journal of Non-Newtonian Fluid Mechanics, 142, 63-78.

Baravian, C., Quemada, D., \& Parker, A. (1996). Modelling thixotropy using a novel structural kinetics approach: basis and application to a solution of iota carrageenan. Journal of Texture Studies, 27, 371-390.

Barnes, H. A. (1997). Thixotropy-a review. Journal of Non-Newtonian Fluid Mechanics, 70, 1-33.

Bautista, F., de Santos, J. M., Puig, J. E., \& Manero, O. (1999). Understanding thixotropic and antithixotropic behavior of viscoelastic micellar solutions and liquid crystalline dispersions. i. the model. Journal of Non-Newtonian Fluid Mechanics, 80, 93-113.

Bautista, F., Fernández, V. V. A., Marcías, E. R., Pérez-López, J. H., Escalante, J. I., Puig, J. E., \& Manero, O. (2012). Experimental evidence of the critical phenomenon and shear banding flow in polymer-like micellar solutions. Journal of Non-Newtonian Fluid Mechanics, 177-178, 89-96.

Bleyer, J., Maillard, M., de Buhan, P., \& Coussot, P. (2015). Efficient numerical computations of yield stress fluid flows using second-order cone programming. Computer Methods in Applied Mechanics and Engineering, 283, 599-614.

Bottino, D. (1998). Modeling viscoelastic networks and cell deformation in the context of the immersed boundary method. Journal of Computational Physics, $147,86-113$.

Bustamante, R., \& Rajagopal, K. R. (2011). Soultions of some simple boundary value problems within the context of a new class of elastic materials. International Journal of Non-Linear Mechanics, 46, 376-386

Chambon, G., Ghemmour, A., \& Naaim, M. (2014). Experimental investigation of viscoplastic free-surface flows in a steady uniform regime. Journal of Fluid Mechanics, 754, 332-364.

Cheddadi, I., \& Saramito, P. (2013). A new operator sppliting algorithm for elastoviscoplastic flow problems. Journal of Non-Newtonian Fluid Mechanics, 202, 13-21.

Chorin, A. (1968). Numerical solution of the Navier-Stokes equations. Mathematics of Computation, 2, 745-762.

Chorin, A., \& Marsden, J. (2000). A mathematical introduction to fluid mechanics. Springer.

Coussot, P. (2014). Yield stress fluid flows: A review of experimental data. Journal of Non-Newtonian Fluid Mechanics, 211, 31-49.

Coussot, P., Nguyen, Q. D., Huynh, H., \& Bonn, D. (2002a). Viscosity bifurcation in thixotropic, yielding fluids. Journal of Rheology, 46, 573-589.

Coussot, P., Nguyen, Q. D., Huynh, H., \& Bonn, D. (2002b). Avalanche behavior in yield stress fluids. Physical Review Letters, 88(175501), 1-4. 
Cuminato, J. A., Oishi, C. M., \& Figueiredo, R. A. (2014). The Impact of Applications on Mathematics. In M. Wakayama, R. S. Andersen, J. Chen, Y. Fukumoto, R. Mckibbin, K. Polthier, T. Tak-agi, \& K.-C. Toh (Eds.), Implicit methods for simulating low Reynolds number free surface flows: Improvements on the MAC-type methods. Japan: Springer.

de Souza Mendes, P. R. (2009). Modeling the thixotropic behavior of structured fluids. Journal of Non-Newtonian Fluid Mechanics, 164, 66-75.

de Souza Mendes, P. R. (2011). Thixotropic elasto-viscoplastic model for structured fluids. Soft Matter, 7, 2471-2483.

de Souza Mendes, P. R., Rajagopal, K. R., \& Thompson, R. L. (2013). A thermodynamic framework to model thixotropic materials. International Journal of Non-Linear Mechanics, 55, 48-54.

de Souza Mendes, P. R., \& Thompson, R. L. (2012). A critical overview of elasto-viscoplastic thixotropic modeling. Journal of Non-Newtonian Fluid Mechanics, 187-188, 8-15.

de Souza Mendes, P. R., \& Thompson, R. L. (2013). A unified approach to model elasto-viscoplastic thixotropic yield-stress materials and apparent yield-stress fluids. Rheologica Acta, 52, 673-694.

Denn, M., \& Bonn, D. (2011). Issues in the flow of yield stress fluids. Rheologica Acta, 50, 307-315.

Derksen, J., \& Regulagadda, P. (2009). Simulations of complex flow of thixotropic liquids. Journal of Non-Newtonian Fluid Mechanics, 160, 65-75.

Dimitriou, C. J., \& McKinley, G. H. (2014). A comprehensive constitutive law for waxy crude oil: a thixotropic yield stress fluid. Soft Matter, 10(35), $6619-6644$.

Dubash, N., Balmforth, N. J., Slima, A. C., \& Cochard, S. (2009). What is the final shape of a viscoplastic slump? Journal of Non-Newtonian Fluid Mechanics, 158, 91-100.

Dullaert, K., \& Mewis, J. (2006). A structural kinetics model for thixotropy. Journal of Non-Newtonian Fluid Mechanics, 139, $21-30$.

Fall, A., Paredes, J., \& Bonn, D. (2010). Yielding and shear banding in soft glassy materials. Physical Review Letters, 105, 225502-1-225502-4.

Fonseca, C., Frey, S., Naccache, M. F., \& de Souza Mendes, P. R. (2013). Flow of an elasto-viscoplastic thixotropic fluid past a confined cylinder. Journal of Non-Newtonian Fluid Mechanics, 193, 80-88.

Frigaard, I., \& Nouar, C. (2005). On the use of viscosity regularisation methods for visco-plastic fluid flow computation. Journal of Non-Newtonian Fluid Mechanics, 127, 1-26.

Glowinsky, R., \& Wachs, A. (2011). On the numerical simulation of viscoplastic fluid flow.. Handbook of Numerical Analysis: 16, pp. 483-717. Elsevier.

Gou, K., Mallikarjuna, M., Rajagopal, K. R., \& Walton, J. R. (2015). Modeling fracture in the context of a strain-limiting theory of elasticity: A single plane-strain crack. International Journal of Engineering Science, 88, 73-82.

Guermond, J., Minev, P., \& Shen, J. (2006). An overview of projection methods for incompressible flows. Computer Methods in Applied Mechanics and Engineering, 195, 6011-6045.

Harlow, F., \& Welch, J. (1965). Numerical calculation of time-dependent viscous incompressible flow of fluid with free surface. Physics of Fluids, 8, $2182-2189$.

Hewitt, D. R., \& Balmforth, N. J. (2013). Thixotropic gravity currents. Journal of Fluid Mechanics, 727, 56-82.

Huynh, H., Roussel, N., \& Coussot, P. (2005). Aging and free surface flow of a thixotropic fluid. Physics of Fluids, 17(033101), 1-9.

I.J.Rao, \& K.R.Rajagopal (2002). A thermodynamic framework for the study of crystallization in polymers. Zeitschrift für angewandte Mathematik und Physik, $53,365-406$.

Kee, D. D., \& Fong, C. (1993). Letter to the editor: A true yield stress? Journal of Rheology, 37, 775-776.

Khajehsaeid, H., Arghavani, J., Naghdabadi, R., \& Sohrabpour, S. (2014). A visco-hyperelastic constitutive model for rubber-like materials: A rate-dependent relaxation time scheme. International Journal of Engineering and Science, 79, 44-58.

Link, F. B., Frey, S., Thompson, R. L., Naccache, M. F., \& de Souza Mendes, P. R. (2015). Plane flow of thixotropic elasto-viscoplastic materials through a 1:4 sudden expansion. Journal of Non-Newtonian Fluid Mechanics, 220, 162-174.

López-Aguilar, J. E., Webster, M. F., Tamaddon-Jahromi, H., \& Manero, O. (2015). Numerical modelling of thixotropic and viscoelastoplastic materials in complex flows. Rheologica Acta, 54, 307-325.

Marrucci, G., Titomanlio, G., \& Sarti, G. C. (1973). Testing of a constitutive equationfor entangled networks by elongational and shear data of polymer melts. Rheologica Acta, 12, 269-275.

Martins, F. P., Oishi, C. M., Afonso, A. M., \& Alves, M. A. (2015). A numerical study of the kernel conformation transformation for transient viscoelastic fluid flows. Journal of Computational Physics, 302, 653-673.

McKee, S., Tomé, M. F., Ferreira, V. G., Cuminato, J. A., Castelo, A., Sousa, F. S., \& Mangiavacchi, N. (2008). The mac method. Comp. Fluids, 37, 907-930.

Mewis, J., \& Wagner, N. J. (2009). Thixotropy. Advance Colloid Interface Science, 147-148, 214-227.

von Mises, R. (1913). Mechanik der festen Körper im plastisch deformablen Zustand. Mathematical Physics, 1, $582-592$.

Mitsoulis, E. (2007). Annular extrudate swell of pseudoplastic and viscoplastic fluids. Journal of Non-Newtonian Fluid Mechanics, 141, 138-147.

Mitsoulis, E., \& Huilgol, R. (2004). Entry flows of Bingham plastics in expansions. Journal of Non-Newtonian Fluid Mechanics, 122, 45-54.

Moller, P., Fall, A., Chikkadi, V., Derks, D., \& Bonn, D. (2009). An attempt to categorize yield stress fluid behavior. Philosophical Transactions Royal Society A, 367, 5139-5155.

Møller, P. C. F., Mewis, J., \& Bonn, D. (2006). Yield stress and thixotropy: on the difficulty of measuring yield stresses in practice. Soft Matter, 2, 274-283.

Mujumdar, A., Beris, A. N., \& Metzner, A. B. (2002). Transient phenomena in thixotropic systems. Journal Non-Newtonian Fluid Mechanics, 102, 157-178.

Muliana, A., Rajagopal, K. R., \& Wineman, A. S. (2013). A new class of quasi-linear models for describing the nonlinear viscoelastic response of materials. Acta Mechanica, 224, 2169-2183.

Nguyen, Q. D., \& Boger, D. V. (1985). Thixotropic behavior of concentrated bauxite residue suspensions. Rheologica Acta, 24, 427-437.

Nigmatullin, R. R., Nakanishi, H., Tran-Cong-Miyata, Q., Tahara, D., \& Fukao, K. (2010). Application of new treatment methods for "reading" of the complex capacitance: A quantitative description of the aging phenomenon in polymer glasses. Communications in Nonlinear Science and Numerical Simulation, 15, 1286-1307.

Oishi, C. M., Martins, F. P., Tomé, M. F., Cuminato, J. A., \& McKee, S. (2011). Numerical solution of the extended pom-pom model for viscoelastic free surface flows. Journal of Non-Newtonian Fluid Mechanics, 166, 165-179.

Oishi, C. M., Tomé, M. F., Cuminato, J. A., \& McKee, S. (2008). An implicit technique for solving 3D low Reynolds number moving free surface flows. Journal of Computational Physics, 227, 7446-7468.

Olmsted, P. (2008). Perspectives on shear banding in complex fluids. Rheologica Acta, 47, 283-300.

Ovarlez, G., Rodts, S., Chateau, X., \& Coussot, P. (2009). Phenomenology and physical origin of shear localization and shear banding in complex fluids. Rheologica Acta, 48, 831-844.

Pashias, N., Boger, D. V., Summers, J., \& Glenister, D. J. (1996). A fifty cent rheometer for yield stress measurement. Journal of Rheol., 40, 1179-1189.

Potanin, A. (2010). 3d simulations of the flow of thixotropic fluids, in large-gap couette and vane-cup geometries. Journal of Non-Newtonian Fluid Mechanics, $165,299-312$.

Poux, A., Glockner, S., \& Azaiez, M. (2012). Improvements on open and traction boundary conditions for Navier-Stokes time-splitting methods. Journal of Computational Physics, 230, 4011-4027.

Quemada, D. (1999). Rheological modelling of complex fluids: IV: Thixotropic and "thixoelastic" behaviour. Start-up and stress relaxation, creep tests and hysteresis cycles. The European Physical Journal Applied Physics, 5(2), 191-208.

Rajagopal, K. R., \& Srinivasa, A. (1998a). Mechanics of the inelastic behavior of materials - part i, theoretical underpinnings. International Journal of Plasticity, 14, 945-967.

Rajagopal, K. R., \& Srinivasa, A. (1998b). Mechanics of the inelastic behavior of materials - part ii, inelastic response. International Journal of Plasticity, 14, 969-995.

Rajagopal, K. R. (2011). Conspectus of concepts of elasticity. Mathematics and Mechanics of Solids, 16, 536-562. 
Rajagopal, K. R., \& Srinivasa, A. R. (2004a). On the thermomechanics of materials that have multiple natural configurations, part 1: Viscoelasticity and Classical Plasticity. Zeitschrift für angewandte Mathematik und Physik, 55, 861-893.

Rajagopal, K. R., \& Srinivasa, A. R. (2004b). On the thermomechanics of materials that have multiple natural configurations, part2: Twinning and Solid to Solid phase transitions. Zeitschrift für angewandte Mathematik und Physik, 55, 1074-1093.

Roussel, N., \& Coussot, P. (2005). "Fifty-cent rheometer" for yield stress measurements: From slump to spreading flow. Journal of Rheology, 49, 705-718.

Sadequi, S., Khabazi, N., \& Sadeghy, K. (2011). Blasius flow of thixotropic fluids: A numerical study. Communications in Nonlinear Science and Numerical Simulation, 16, 711-721.

dos Santos, D. D., Frey, S. L., Naccache, M. F., \& de Souza Mendes, P. R. (2014). Flow of elasto-viscoplastic liquids through a planar expansion-contraction. Rheologica Acta, 53, 31-41.

dos Santos, T., Ramos, G. R., \& Rossi, R. (2015). A note on overstress and over-thermodynamic forces derivation for elasto-viscoplastic media: Thermodynamic analysis of an isothermal relaxation process. International Journal of Engineering Science, 93, 13-30.

Soares, E. J., Thompson, R. L., \& Machado, A. (2013). Measuring the yielding of waxy crude oils considering its time-dependency and apparent-yield-stress nature. Applications of Rheology, 23(62798), 1-11.

Staron, L., Lagrée, P. Y., Ray, P., \& Popinet, S. (2013). Scaling laws for the slumping of a Bingham plastic fluid. Journal of Rheology, 57, 1265-1280.

Tarcha, B. A., Forte, B. P. P., Soares, E. J., \& Thompson, R. L. (2015). Critical quantities on the yielding process of waxy crude oils. Rheologica Acta, 54, 479-499.

Temam, R. (1969). Sur l'approximation de la solution des equations de Navier-Stokes par la méthode de pas fractionnaires (ii). Archive for Rational Mechanics and Analysis, 33, 377-385.

Thompson, R. L., \& Soares, E. J. (2016). Viscoplastic dimensionless numbers. Journal of Non-Newtonian Fluid Mechanics. doi:10.1016/j.jnnfm.2016.05.001.

Tryggvason, G., Scardovelli, R., \& Zaleski, S. (2011). Direct numerical simulations of gas-liquid multiphase flows. Cambridge University Press.

Van Der Geest, C., Guersoni, V. C. B., Merino-Garcia, D., \& Bannwart, A. C. (2015). A modified elasto-viscoplastic thixotropic model for two commercial gelled waxy crude oils. Rheologica Acta, 54, 545-561.

Vola, D., Boscardin, L., \& Latché, J. (2003). Laminar unsteady flows of Bingham fluids: a numerical strategy and some benchmark results. Journal of Computational Physics, 187, 441-456.

Yziquel, F., Carreau, P., Moan, M., \& Tanguy, P. (1999). Rheological modeling of concentrated colloidal suspensions. Journal of Non-Newtonian Fluid Mechanics, $86,133-155$. 